Conectividade para um modelo de grafo aleatório não homogêneo

Eduardo Zorzo Sartoretto 

SERVIÇO DE PÓS-GRADUAÇÃO DO ICMC-USP

Data de Depósito:

Assinatura:

Eduardo Zorzo Sartoretto

\title{
Conectividade para um modelo de grafo aleatório não homogêneo
}

\begin{abstract}
Dissertação apresentada ao Instituto de Ciências Matemáticas e de Computação - ICMC-USP, como parte dos requisitos para obtenção do título de Mestre em Ciências - Ciências de Computação e Matemática Computacional. VERSÃO REVISADA

Área de Concentração: Ciências de Computação e Matemática Computacional

Orientador: Prof. Dr. Pablo Martín Rodríguez

Coorientador: Prof. Dr. Francisco

Aparecido Rodrigues
\end{abstract}

Maio de 2016 
Ficha catalográfica elaborada pela Biblioteca Prof. Achille Bassi e Seção Técnica de Informática, ICMC/USP, com os dados fornecidos pelo(a) autor(a)

Sartoretto, Eduardo Zorzo
Conectividade para um modelo de grafo aleatório
não homogêneo / Eduardo Zorzo Sartoretto; orientador
Pablo Martín Rodriguez; coorientador Francisco
Aparecido Rodrigues. -- São Carlos -- SP, 2016.
110 p.
Dissertação (Mestrado - Programa de Pós-Graduação
em Ciências de Computação e Matemática Computacional)
-- Instituto de Ciências Matemáticas e de Computação,
Universidade de São Paulo, 2016.
1. Grafos aleatórios. 2. Modelo de Erdös-Rényi.
3. Conectividade de grafos. I. Rodríguez, Pablo Martín,
orient. II. Rodrigues, Francisco Aparecido, coorient.
III. Título.


Eduardo Zorzo Sartoretto

Connectivity to an inhomogeneous random graph model

Master dissertation submitted to the Instituto de Ciências Matemáticas e de Computação - ICMC-USP, in partial fulfillment of the requirements for the degree of the Master Program in Computer Science and Computational Mathematics. FINAL VERSION

Concentration Area: Computer Science and Computational Mathematics

Advisor: Prof. Dr. Pablo Martín Rodríguez

Co-advisor: Prof. Dr. Francisco Aparecido Rodrigues

USP - São Carlos

May 2016 

Aos meus familiares, amigos e em especial à minha esposa Bruna. 

Primeiramente à Deus, pelo dom da vida e pelas graças concedidas.

À minha esposa Bruna, pelo apoio e dedicação realizados principalmente nestes dois últimos anos, ajudando e dando forças em momentos difíceis, sofrendo junto e também compartilhando momentos de alegria.

Ao meu orientador Pablo M. Rodríguez, pela paciência, esforço, otimismo e orientação neste projeto.

Aos meus familiares, que me incentivaram, motivaram e aconselharam mesmo estando a vários quilômetros de distância. Sou grato por tudo que fizeram e fazem por mim até hoje.

Aos meus antigos amigos de longe (Kinho, Déia, Thiago, Morgana, Flávia, Ellene, Lino, Paula, Zé, Carla, Ronaldo, Alessandra, Cleyton, Brunella, Michel, ...), os novos amigos de perto (Felipe, Carol, Marcão, Luciana, Joaquim, Vitor, Ana, ...) e aos de longe que estão perto (Guilherme e Juliana), por se tornarem uma segunda família em vários momentos, partilhando suas alegrias, anseios e dúvidas. Mesmo com as constantes mudanças da vida, a amizade permanecerá.

Aos professores Renato Gava e Francisco A. Rodrigues, que tornaram possível a realização deste trabalho fornecendo seus conhecimentos.

Ao pessoal da secretaria acadêmica, Leonardo, Alexandre e Ana, pela incrível disposição, ajudando em todos os problemas que lhes cabem da melhor forma possível.

A todos que contribuíram, direta ou indiretamente para a realização deste trabalho.

Ao $\mathrm{CNPq}$, pelo apoio financeiro, indispensável durante este período. 

"Por vezes sentimos que aquilo que fazemos não é senão uma gota de água no mar, mas o mar seria menor se lhe faltasse uma gota." (Madre Teresa de Calcuta) 



\section{RESUMO}

SARTORETTO, E. Z.. Conectividade para um modelo de grafo aleatório não homogêneo. 2016. 110 f. Dissertação (Mestrado em Ciências - Ciências de Computação e Matemática Computacional) - Instituto de Ciências Matemáticas e de Computação (ICMC/USP), São Carlos - SP.

A caracterização de redes e o estudo de sistemas, ambos utilizando grafos, é algo muito usado por várias áreas científicas. Uma das linhas deste estudo é denominada de grafos aleatórios, que por sua vez auxilia na criação de modelos para análise de redes reais.

Consideramos um modelo de grafo aleatório não homogêneo criado por Kang, Pachón e Rodríguez (2016), cuja construção é feita a partir da realização do grafo binomial $G(n, p)$. Para este modelo, estudamos argumentos e métodos usados para encontrar resultados sobre o limiar de conectividade, importante propriedade relacionada a existência assintótica de vértices e componentes isolados. Em seguida, constatamos algumas características positivas e negativas a respeito da utilização do grafo para modelar redes reais complexas, onde usamos de simulações computacionais e medidas topológicas.

Palavras-chave: Grafos aleatórios, Modelo de Erdös-Rényi, Conectividade de grafos. 



\section{ABSTRACT}

SARTORETTO, E. Z.. Conectividade para um modelo de grafo aleatório não homogêneo. 2016. 110 f. Dissertação (Mestrado em Ciências - Ciências de Computação e Matemática Computacional) - Instituto de Ciências Matemáticas e de Computação (ICMC/USP), São Carlos - SP.

The characterization of networks and the study of systems, both using graphs, is very used by several scientific areas. One of the lines of this study is called random graphs, which in turn assists in creating models for the analysis of real networks.

We consider an inhomogeneous random graph model created by Kang, Pachón e Rodríguez (2016), where its construction is made from the realization of the binomial graph $G(n, p)$. For this model, we studied the arguments and methods used to find results on the connectivity threshold, important property related to asymptotic existence of vertices and isolated components. Then we found some positive and negative characteristics about the use of the graph to model complex real networks, using computer simulations and topological measures.

Key-words: Random graphs, Erdös-Rényi model, connectivity threshold of graphs. 



\section{LISTA DE ILUSTRAÇÕES}

Figura 1 - Ilustração das pontes de Königsberg . . . . . . . . . . . . . . . 26

Figura 2 - Exemplos de grafos. . . . . . . . . . . . . . . 27

Figura 3 - Exemplo para isomorfismo. . . . . . . . . . . . . 27

Figura 4 - Grafo com matriz de adjacência. . . . . . . . . . . . . . 28

Figura 5 - Graus para o grafo G. . . . . . . . . . . . . . . . . . 29

Figura 6 - Exemplo de grafo e subgrafo. . . . . . . . . . . . . . . . 29

Figura 7 - Passeios, caminhos e ciclos. . . . . . . . . . . . . 30

Figura 8 - Grafos conectados e desconectados. . . . . . . . . . . . . . 30

Figura 9 - Exemplo de componentes, note que $G=H_{1} \cup H_{2} \cup H_{3} \ldots \ldots$. . . . . . . 31

Figura 10 - Exemplo de floresta e árvore. . . . . . . . . . . . . . . . . 31

Figura 11 - As 3 possíveis árvores de 3 vértices. . . . . . . . . . . . . . . 32

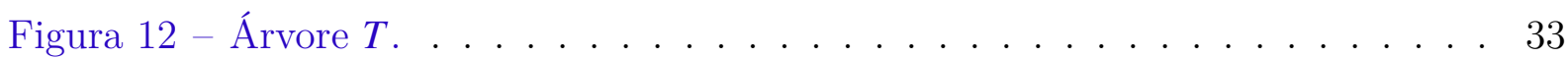

Figura 13 - (a)Grafo nulo com 15 vértices. (b) Realização do modelo binomial $G(15,0.1)$. (c) Realização do modelo binomial $G(15,0.4)$. . . . . . . . 35

Figura 14 - Diagrama das convergências . . . . . . . . . . . . . . 38

Figura 15 - (a) Iniciamos com um conjunto de $n$ vértices. (b) Criamos um grafo binomial $G(n, p) \ldots \ldots \ldots \ldots$. . . . . . . . . . . . 52

Figura 16 - (c) Estabelecemos uma configuração de comunidades de forma arbitrária. (d) Criamos super-vértices com as comunidades e os conectamos de acordo com a conexão anterior em $G(n, p)$. . . . . . . . . . . . 52

Figura 17 - Função $f(x)$ com $J=5$ e $N=8 \ldots \ldots$. . . . . . . . . . . . . . . . . 61

Figura 18 - Função $\mathrm{f}(\mathrm{x})=\mathrm{x}(\mathrm{n}-\mathrm{x})$, para $n=8, x \in(0, n) \ldots \ldots \ldots 7$

Figura 19 - Distribuições dos graus . . . . . . . . . . . . . . . 77

Figura 20 - Distribuições dos graus . . . . . . . . . . . . . . . . . . 77

Figura 21 - Distribuição acumulada dos coeficientes de aglomeração locais . . . . . 78

Figura 22 - Distribuições dos Betweenness . . . . . . . . . . . . . . . . 78

Figura 23 -Graficos $k \times k_{n n} \ldots \ldots \ldots \ldots \ldots \ldots$

Figura 24 - Distribuições dos graus . . . . . . . . . . . . . . . . . . . . 80

Figura 25 - Distribuições dos graus . . . . . . . . . . . . . . . . . . . 80

Figura 26 - Distribuição acumulada dos coeficientes de aglomeração locais . . . . . 81

Figura 27 - Distribuições dos Betweenness . . . . . . . . . . . . . . . . . . 81

Figura 28 -Graficos $k \times k_{n n} \ldots \ldots \ldots \ldots \ldots \ldots$. . . . . . . . . . . . . . . .

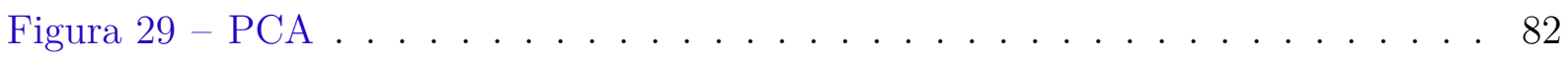




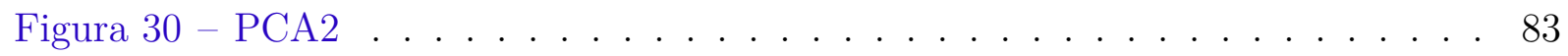

Figura 31 - Modelo de Histograma . . . . . . . . . . . . . . . . . . . . . . . 99

Figura 32 - Exemplos de cálculos da entropia normalizada . . . . . . . . . . . 100

Figura 33 - Exemplos para aglomerações . . . . . . . . . . . . . . . . . . . . . . 101

Figura 34 - Transitividades . . . . . . . . . . . . . . . . . . . . . . . 102

Figura 35 - Coeficientes de correlação locais . . . . . . . . . . . . . . . . . 102

Figura 36 - Caminhos Geodésicos . . . . . . . . . . . . . . . . . . . 103

Figura 37 - Betweenness . . . . . . . . . . . . . . . . . . . . . 105

Figura 38 - Exemplos de gráficos $k \times k_{n n} \ldots \ldots \ldots$. . . . . . . . 106 
$G(n, p)$ — Grafo binomial de $n$ vértices com probabilidade de conexão $p$

$G(N, \mathcal{K}, p)$ - Grafo aleatório do modelo de Kang, Pachón e Rodríguez

$I_{A}$ - Variável aleatória indicadora do evento $A$

$\operatorname{Bin}(n, p)$ - Distribuição binomial com parâmetros $n$ e $p$

$\operatorname{Poi}(\lambda)$ - Distribuição de Poisson com parâmetro $\lambda$

$d_{T V}(p, q)$ - Distância de variação total entre as funções $p$ e $q$

$O(x)$ - Uma função $f(x)$ é dita $O(x)$ se existem duas constantes positivas $c$ e $x_{0}$ tais que $|f(x)| \leq c|g(x)| \forall x>x_{0}$

$\mathbb{R}$ — Conjunto dos números reais

$\mathbb{N}$ - Conjunto dos números naturais

$\approx$ - Aproximado

$\left(\begin{array}{l}n \\ m\end{array}\right)$ — combinação de $n$ elementos tomados $m$ a $m$

$A_{k_{i}, 2}$ - Arranjo de $k_{i}$ elementos tomados 2 a 2 

INTRODUÇÃO ........................ 21

ALGUNS CONCEITOS DE TEORIA DOS GRAFOS . . . . . . . 25

MÉTOdOS PROBABILÍSTICOS . . . . . . . . . . . . . . . . . . 37

3.1 Convergência de variáveis aleatórias . . . . . . . . . . . . . . 37

3.2 Acoplamento e Método de Stein . . . . . . . . . . . . . . . 44

3.3 Desigualdades probabilísticas . . . . . . . . . . . . . 49

4 MODELO de GRAFO ALEATÓRIO NÃO HOMOGÊNEO . . . . . 51

$4.1 \quad$ Construção do modelo . . . . . . . . . . . . . . . 51

4.2 Resultados sobre conectividade . . . . . . . . . . . . 53

4.3 Momentos do número de super-vértices isolados . . . . . . . . . . 54

4.4 Existência de super-vértices isolados . . . . . . . . . . . . 58

4.5 Distribuição do número de super-vértices isolados . . . . . . . . . 62

4.6 Componentes pequenos . . . . . . . . . . . . . 65

4.7 Prova do Teorema de Conectividade . . . . . . . . . . . . 71

5 SIMULAÇÕES E RESULTADOS . . . . . . . . . . . . . 73

5.1 Métodos de simulação . . . . . . . . . . . . . . 73

$5.2 \quad$ Simulações para duas redes reais . . . . . . . . . . . . . . 74

6 CONCLUSÃO E DISCUSSÕES . . . . . . . . . . . . . 85

REFERÊNCIAS . . . . . . . . . . . . . . . . . . . 87

APÊNDICE A SÉRIES DE TAYLOR E MACLAURIN . . . . . . 91

APÊNDICE B NOTAÇÃO ASSINTÓTICA E FÓRMULA DE STIRLING . . . . . . . . . . . . . . . . . . . 93 
ANEXO A

CÓDIGOS PARA SIMULAÇÕES NO SOFTWARE R . . 107 
Ao nosso redor existem vários conjuntos de elementos que podem se conectar por meio de uma relação binária. Somos capazes de interpretar estes conjuntos como uma espécie de "rede", onde os nós são os elementos e as ligações entre eles são estabelecidas se a relação for positiva. Como exemplo podemos considerar a internet, sendo ela um sistema de iterações entre computadores, formando assim uma rede física. Além desta ocasião, muitas outras situações podem ser convenientemente descrita por meio de diagramas que consistem em um conjunto de pontos que são ligados por arestas. Estes diagramas em formato de redes são chamados de grafos e a abstração matemática para situações deste tipo acaba remetendo aos conceitos da teoria dos grafos (LUCCHESI, 1979).

As possibilidades de modelagem de sistemas por meio de grafos são enormes. Como exemplos podemos pensar em cidades que são relacionadas caso haja uma estrada que as conecte; pesquisadores relacionados caso haja uma colaboração cientifica entre eles; páginas de internet relacionadas caso haja algum link que as conecte; elementos químicos relacionados por suas reações químicas; aeroportos relacionados casa haja alguma linha de passageiros que os conecte. Devido a esta versatilidade de aplicações, a teoria dos grafos pode ser aplicada a uma ampla gama de áreas científicas como física, química, biologia, ciência da computação, informática, engenharia elétrica, engenharia civil, arquitetura, pesquisa operacional, psicologia, sociologia, economia, antropologia e linguística. E também esta intimamente relacionada com muitos ramos da matemática, entre eles a teoria dos grupos, teoria de matrizes, análise numérica, probabilidade, topologia e análise combinatória (HARARY, 1969).

A teoria dos grafos aleatórios é uma subárea da teoria dos grafos que se refere à pesquisa envolvendo propriedades de grafos criados a partir de processos aleatórios. Ela se iniciou no final dos anos de 1950 com estudos desenvolvidos por Paul Erdös e Alfred Rényi. Posteriormente, em meados do século XX, Watts e Strogatz (1998) criaram um modelo 
denominado pequeno mundo e Albert e Barabási (1999) estipularam outro modelo usando de uma fixação preferencial (resumidamente, estipula-se uma quantia inicial de vértices e conexões, em seguida inicia-se um processo de inclusão de novos vértices conectados aos já existentes dando preferência a uma propriedade específica pré-estipulada), o que acabou ocasionando uma explosão de investigações nesta área de pesquisa, tanto que no inicio de julho de 2005 o Science Citation Index informou que haviam 1154 citações de Watts e Strogatz e 964 de Barabási e Albert (DURRETT, 2007).

Um grande número de artigos foram publicados nas revistas Science e Nature, onde um deles fez a seguintes afirmação: "A internet é robusta, mas frágil. 95\% das ligações na internet podem ser removidas e ainda sim haverá distribuição de internet para algumas conexões. No entanto, a remoção de 2,3\% de ligações específicas acarretaria no desligamento completo da internet." Esta declaração é muito interessante, entretanto, algum tempo depois foi constatado que ela não é totalmente correta (DURRETT, 2007). O fato para nós não é saber a verdade sobre esta afirmação, mas sim como é feita a análise deste tipo de problema. Como muitas redes reais ainda possuem uma grande dificuldade de serem catalogadas, isto é, determinadas por completo, é comum a utilização de grafos aleatórios com o intuito de modelar tais redes. Desta maneira, se o modelo de grafo aleatório proporcionar uma boa semelhança com a rede, as propriedades e características usadas ou já conhecidas para o grafo formado podem ser consideradas como também existentes na rede real, auxiliando assim em seu entendimento. $\mathrm{O}$ que nos remete à importância em conhecer e desenvolver resultados, sobre o máximo possível de características para cada modelo de grafo aleatório.

O primeiro modelo de grafo aleatório desenvolvido por Erdös e Rényi (1959) foi construído distribuindo uma quantia $M$ fixa de arestas entre as possíveis conexões entre outra quantia de vértices $n$, também pré-estabelecidas, fazendo com que a notação de um grafo deste modelo fosse $G(n, M)$. Gilbert (1959) estabeleceu outro modelo equivalente, mas que utiliza de $n$ vértices conectados uns com os outros usando uma probabilidade $p$. O modelo foi chamado de modelo binomial e utiliza a notação $G(n, p)$. Uma propriedade encontrada nestes dois modelos é a homogeneidade, ela indica que a probabilidade em relação ao número de conexões para cada vértice é a mesma, diferentemente do modelo proposto por Barabási e Albert comentado anteriormente. Neste quesito a homogeneidade não é vantajoso para modelar redes reais, pois é conhecido que a maioria delas tem a característica de possuírem probabilidades de conexão fortemente distintas, exemplos podem ser vistos em Boccaletti et al. (2006, p. 188) e Newman (2003, p. 10).

Em Kang, Pachón e Rodríguez (2016) é proposto um novo modelo que aborda a criação de um grafo aleatório a partir do modelo binomial $G(n, p)$, utilizando de comunidades que são denominados super-vértices. Este modelo é descrito na forma $G(N, \mathcal{K}, p)$, onde $N$ denota o número de super-vértices com configuração $\mathcal{K}=\left\{k_{i} ; k_{i} \in \mathbb{N}, 1 \leq i \leq r, r \in \mathbb{N}\right\}$, 
com $k_{i}$ denotando o número de super-vértices de tamanho $i$, e $p$ a probabilidade de ligação entre os vértices das comunidades (super-vértices). Neste caso é possível estabelecer a propriedade de não homogeneidade de acordo com a escolha dos parâmetros do modelo. Esta transformação de característica traz algo muito interessante ao modelo, principalmente no que se refere a modelagem. No artigo, os autores também apresentam resultados sobre o limiar de conectividade, existência de um componente gigante e a distribuição do grau de um vértice.

O objetivo deste projeto de mestrado é estudar os argumentos e métodos utilizados para comprovar uma importante propriedade relacionada ao limiar de conectividade do modelo $G(N, \mathcal{K}, p)$, por meio de um teorema. E também realizar algumas simulações com propósito de modelar duas redes reais, onde vamos empregar medidas topológicas para verificar características positivas e negativas do modelo em relação a modelagem de redes.

A estrutura do trabalho é composta da seguinte forma:

- No capítulo 2, é apresentado uma revisão bibliográfica sobre a teoria dos grafos, utilizada para auxiliar na reprodução e entendimento do modelo proposto, e também para comprovar o principal teorema sobre conectividade.

- No capítulo 3, é apresentado uma revisão bibliográfica de alguns métodos probabilísticos com seus respectivos teoremas resultados e definições. Tais métodos irão ajudar na comprovação do teorema principal, ou mesmo fornecer ideias diferentes para sua prova.

- No capítulo 4, é apresentada a definição do modelo $G(N, \mathcal{K}, p)$, o principal teorema de estudo que envolve a conectividade assintótica e a sua demonstração a partir de passos envolvendo o comportamento de uma variável aleatória que designa o número de vértices isolados.

- No capítulo 5, são apresentadas três formas de reproduzir o modelo e algumas propriedades resultantes de simulações feitas para duas redes reais.

- No capítulo 6, é apresentado a conclusão juntamente com discussões sobre os resultados obtidos das simulações e algumas ideias para futuros projetos. 



\section{ALGUNS CONCEITOS DE TEORIA DOS GRAFOS}

A teoria dos grafos se desenvolveu através de problemas envolvendo jogos e quebracabeças, que chamavam cada vez mais a atenção por seus resultados teóricos aprofundados e variados (COSTA, 2011). Um estudo interessante feita sobre a relação dos grafos e teoria dos jogos é apresentado em Gonçalves (2007). De fato, a primeira menção registrada do assunto ocorreu nas obras do matemático suíço Leonhard Euler (1707-1783), que em 1736 escreveu um artigo discutindo sobre um enigma, conhecido atualmente como O Problema das Pontes de Königsberg (COSTA, 2011). Este problema foi desenvolvido a partir das sete pontes que estabelecem ligações entre as margens do Rio Pregel e duas de suas ilhas, ilustrado na Figura 1. O objetivo consiste em, a partir de um determinado ponto, passar por todas as pontes somente uma vez e retornar ao ponto inicial.

Subsequentemente houveram mais descobertas feitas por Gustav Robert Kirchhoff (1824-1887), onde suas investigações a respeito de redes elétricas levaram ao desenvolvimento de conceitos básicos e teoremas relativos a árvores em grafos, e por Arthur Cayley (1821-1895), que considerou grafos decorrentes da enumeração de isômeros em química (HARARY, 1969). Um problema que merece ser mencionado e que foi apenas resolvido com ajuda computacional é o Problema das Quatro Cores, que trata da determinação do número mínimo de cores necessárias para colorir um mapa, de países reais ou imaginários, de forma a que países com fronteira comum tenham cores diferentes. É possível relacionar este problema com um grafo onde os pontos são os países e as relações (ligações) são estabelecidas se existe uma fronteira em comum (SOUSA, 2001).

Os conceitos aqui apresentados foram obtidos de Diestel (2000), Carvalho (2005), Costa (2011), Harju (2011) e Sudakov (2015). 


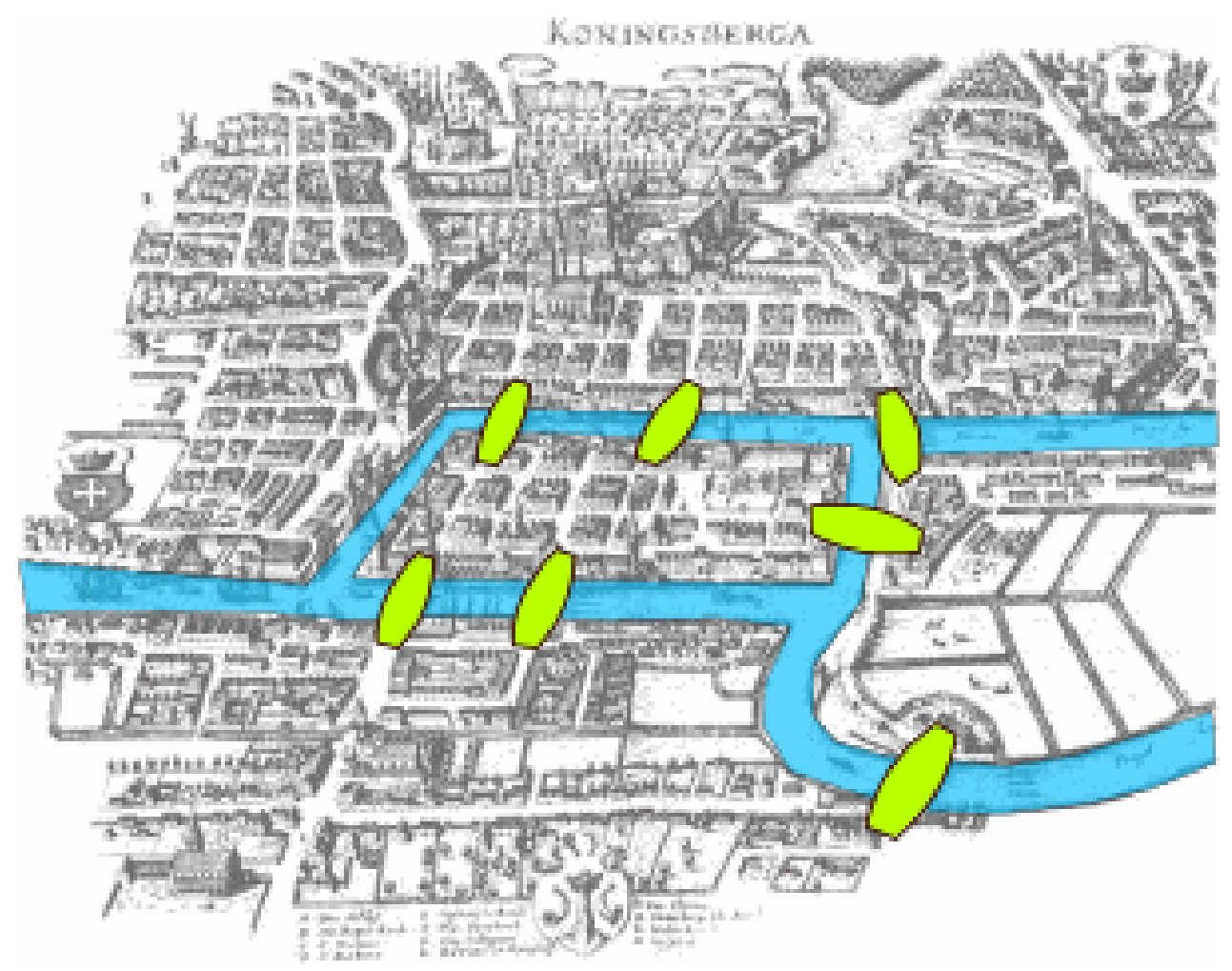

Figura 1 - Ilustração das pontes de Königsberg

Fonte: https://en.wikipedia.org/wiki/Seven_Bridges_of_Königsberg

\subsection{Grafos}

Definição 2.1.1. Um grafo $G$ é formado por um par $(V, E)$ onde $V$ é um conjunto de vértices (nós, pontos) e $E$ é um multi-conjunto de pares de vértices não ordenados, ou seja, $E=\{(u, v) ; u, v \in V\}$. Os elementos de $E$ são chamados de arestas. Nós escrevemos $V(G)$ representando o conjunto dos vértices e $E(G)$ representando o conjunto das arestas de $G$. Denotamos também $|G|=|V(G)|$ como o número de vértices e $e(G)=|E(G)|$ como o número de arestas do grafo $G$.

A forma usual de representar um grafo como uma imagem é desenhando pontos que representam os vértices, e ligando dois destes pontos com uma linha que representa a aresta que compõe estes vértices. Caso não houver linha entre dois vértices, estes não são conectados, isto é, não existe aresta que compõe estes vértices, veja Figura 2.

Definição 2.1.2. Um loop é uma aresta $(v, v)$ para algum $v \in V$. Uma aresta $e=(u, v)$ é uma aresta múltipla se ela aparecer mais que uma vez em $E$. Um grafo é dito simples se não possuir loops nem arestas múltiplas. Grafos não simples são comumente chamados de grafos múltiplos.

Definição 2.1.3. Dizemos que:

- vértices $u$ e $v$ são adjacentes ou vizinhos em $V$ se $(u, v) \in E$; 
- uma aresta $e \in E$ é incidente a um vértice $v \in V$ se $v \in e$;

- $\operatorname{arestas} e, e^{\prime}$ são incidentes se $e \cap e^{\prime} \neq \emptyset$.

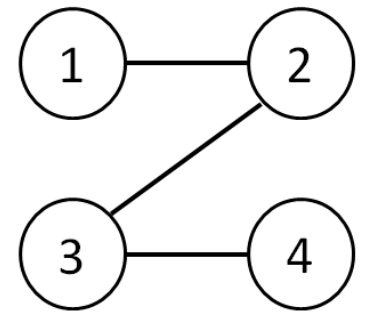

$\mathrm{G}_{1}$

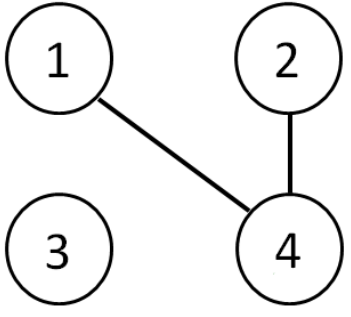

$\mathrm{G}_{2}$

Figura 2 - Exemplos de grafos.

Fonte: Elaborada pelo autor.

Um grafo pode ser estabelecido entre qualquer conjunto de objetos onde possamos definir uma relação de existência comum entre eles. Como por exemplo, podemos definir $V$ como um conjunto de pessoas e $E$ como a existência de ligação parentesca entre essas pessoas (grafo conhecido como rede social), outro exemplo, seja $V$ o conjunto de cidades de um estado e $E$ a relação que informa se existe uma estrada que liga uma cidade a outra (chamado de rede física). Muitos outros exemplos podem ser vistos em Boccaletti et al. (2006, p. 79).

Definição 2.1.4. Sejam $G_{1}=\left(V_{1}, E_{1}\right)$ e $G_{2}=\left(V_{2}, E_{2}\right)$ grafos. Um isomorfismo $\phi: G_{1} \rightarrow$ $G_{2}$ é uma bijeção para $V_{1}$ e $V_{2}$ tal que $(u, v) \in E_{1}$ se, e somente se, $(\phi(u), \phi(v)) \in E_{2}$. Dizemos que $G_{1}$ é isomorfo a $G_{2}$ se existe um isomorfismo entre eles.

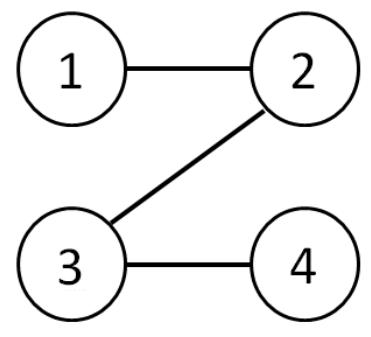

$\mathrm{G}_{1}$

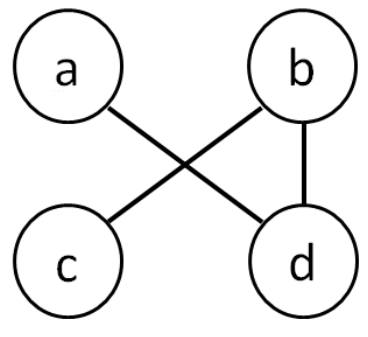

$\mathrm{G}_{2}$

Figura 3 - Exemplo para isomorfismo.

Fonte: Elaborada pelo autor.

Um exemplo pode ser feito considerando a Figura 3, onde podemos estabelecer a função $\phi: G_{1} \rightarrow G_{2}$, dada por $\phi(1)=a, \phi(2)=d, \phi(3)=b, \phi(4)=c$, que é um isomorfismo. 
Um isomorfismo é uma relação de equivalência entre grafos. Isto quer dizer que é uma relação que possuem as seguintes propriedades:

- Todo grafo é isomorfo a ele mesmo;

- se $G_{1}$ é isomorfo a $G_{2}$ então $G_{2}$ é isomorfo a $G_{1}$;

- se $G_{1}$ é isomorfo a $G_{2}$ e $G_{2}$ é isomorfo a $G_{3}$, então $G_{1}$ é isomorfo a $G_{3}$.

Definição 2.1.5. Sejam $B=\{1,2, \ldots, n\}$ com $n \in \mathbb{N}$ e $G=(V, E)$ um grafo com $V=B$. A matriz de adjacência $A=A(G)=\left\{a_{i j}\right\}$ é uma matriz simétrica $n \times n$ definida por:

$$
a_{i j}= \begin{cases}1 & \text { se }(i, j) \in E \\ 0 & \text { caso contrário }\end{cases}
$$

Um exemplo pode ser visto na Figura 4, considere $A=A(G)$.

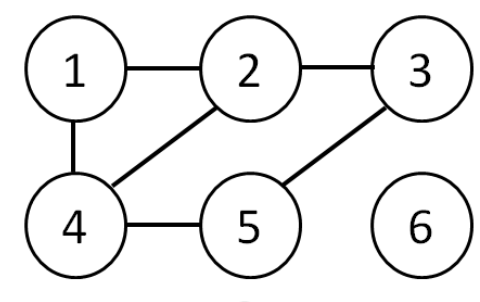

$$
A=\left[\begin{array}{llllll}
0 & 1 & 0 & 1 & 0 & 0 \\
1 & 0 & 1 & 1 & 0 & 0 \\
0 & 1 & 0 & 0 & 1 & 0 \\
1 & 1 & 0 & 0 & 1 & 0 \\
0 & 0 & 1 & 1 & 0 & 0 \\
0 & 0 & 0 & 0 & 0 & 0
\end{array}\right]
$$

G

Figura 4 - Grafo com matriz de adjacência.

Fonte: Elaborada pelo autor.

Definição 2.1.6. Dado $G=(V, E)$ e um vértice $v \in V$, definimos a vizinhança $N(v)$ como sendo o conjunto dos vizinhos do vértice $v$. O grau $d(v)$ de um vértice $v$ é definido como o número de vizinhos de $v$, ou seja, $d(v)=|N(v)|$. Um vértice $v$ é dito isolado se $d(v)=0$.

Note que $d(v)$ pode ser obtido pela soma dos valores da linha correspondente a $v$ na matriz de adjacência $A(G)$.

Definição 2.1.7. Um grafo $G$ é dito $d$-regular se, e só se, todos os vértices do grafo possuem grau $d$.

Proposiçao 2.1.1. Para qualquer grafo $G=(V, E)$, temos que

$$
\sum_{v \in G} d(v)=2|E(G)|
$$

Demonstração. Note que no somatório $\sum_{v \in G} d(v)$ cada aresta $e=(u, v)$ é contado duas vezes, uma para $u$ e outra para $v$. 


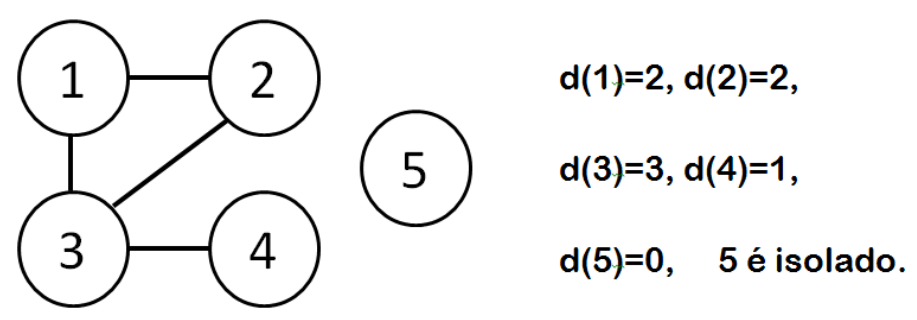

G

Figura 5 - Graus para o grafo $G$.

Fonte: Elaborada pelo autor.

Definição 2.1.8. Sejam dois grafos $G=(V(G), E(G))$ e $H=(V(H), E(H))$.

- Dizemos que $H$ é um subgrafo de $G$ se $V(H) \subseteq V(G)$ e $E(H) \subseteq E(G)$.

- A reunião $J=G \cup H$ é um grafo com o conjunto de vértices $V(J)=V(G) \cup V(H)$ e a família de arestas $E(J)=E(G) \cup E(H)$.

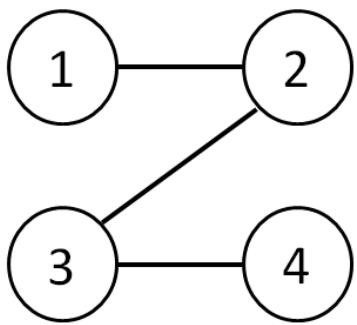

G

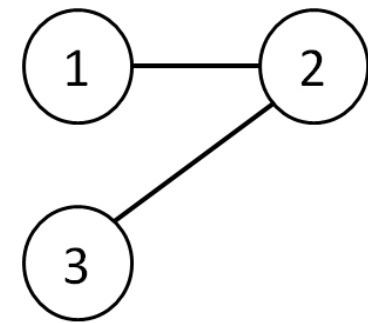

subgrafo de $\mathrm{G}$

Figura 6 - Exemplo de grafo e subgrafo.

Fonte: Elaborada pelo autor.

Definição 2.1.9. Se um grafo $G$ não possui arestas, isto é, $E(G)=\emptyset$, então ele é dito nulo e seus vértices são todos isolados. Já um grafo $G$ é dito completo se, e só se, para quaisquer $u, v \in V$ temos que $(u, v) \in E$.

Definição 2.1.10. Um passeio em $G$ é uma sequência de vértices $v_{0}, v_{1}, \ldots, v_{k}$ e uma sequência de arestas $\left(v_{i}, v_{i+1}\right) \in E(G)$. Um passeio é um caminho se todos os $v_{i}$ 's são distintos. Se, por um tal caminho com $k \geq 2$, a aresta $\left(v_{0}, v_{k}\right)$ também está inclusa em $E(G)$, então $v_{0}, v_{1}, v_{2}, \ldots, v_{k}$ é um ciclo. Para grafos múltiplos, consideramos loops e pares de arestas múltiplas também como sendo ciclos.

Definição 2.1.11. O comprimento de um caminho, passeio ou ciclo é o número de arestas que o compõe, (veja Figura 7). 


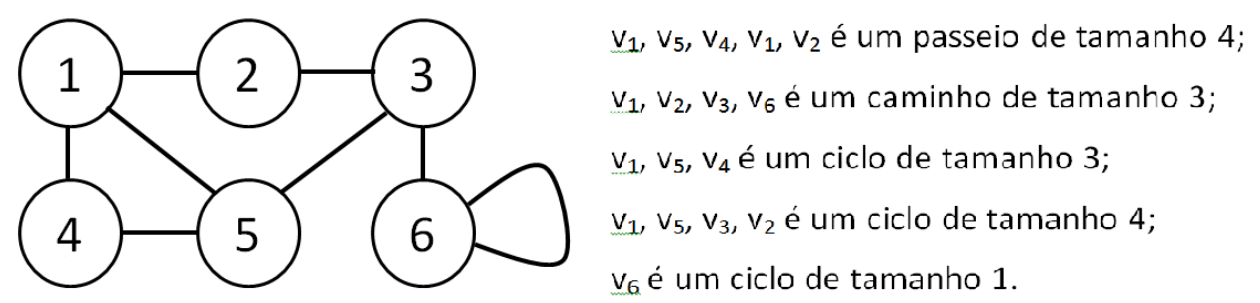

G

Figura 7 - Passeios, caminhos e ciclos.

Fonte: Elaborada pelo autor.

Pode se verificar pelas definições que caso exista um passeio de $u$ para $v$ em um grafo $G$, então existe um caminho entre $u$ e $v$ em $G$.

Definição 2.1.12. Um grafo $G$ é dito conectado se para todo par $u, v \in V(G)$, existe um caminho em $G$ de $u$ para $v$. Caso o grafo não seja conectado dizemos que ele é desconectado.

Esta definição anterior é muito importante em nosso trabalho, pois será o principal objeto de estudo no modelo de grafo apresentado no Capítulo 4.

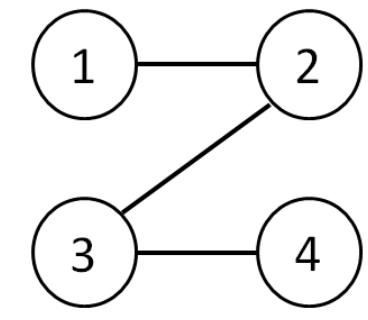

Grafo conectado

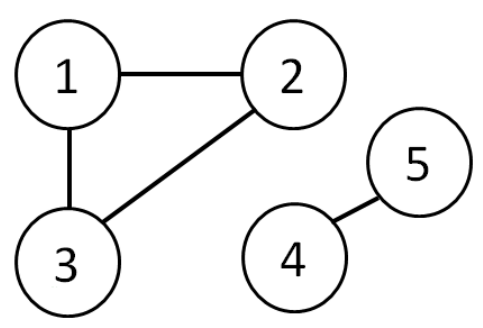

Grafo desconectado

Figura 8 - Grafos conectados e desconectados.

Fonte: Elaborada pelo autor.

Definição 2.1.13. Um componente $H_{1}$ de um grafo $G$ é um subgrafo conectado que compõe uma reunião disjunta de $G$, ou seja, se $G=H_{1} \cup H_{2}, H_{1} \cap H_{2}=\emptyset$ e $H_{1}$ é um grafo conectado então $H_{1}$ é um componente de $G$. Logo, podemos dizer que um grafo é conectado se, e só se, possui apenas um componente, para um exemplo veja Figura 9.

Definição 2.1.14. Seja $G=(V, E)$, o complementar $\bar{G}$ de $G$ é um grafo que possui o mesmo conjunto de vértices $V$, mas $(u, v) \in E(\bar{G})$ se, e só se, $(u, v) \notin E(G)$.

Definição 2.1.15. Um clique de $G$ é um subgrafo completo em $G$ e um conjunto independente é um subgrafo nulo em $G$. 


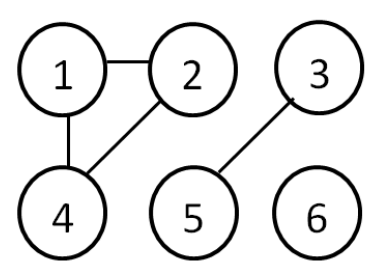

G

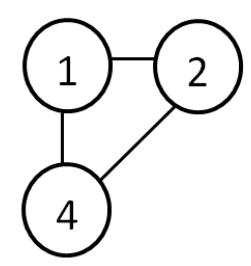

$\mathrm{H}_{1}$

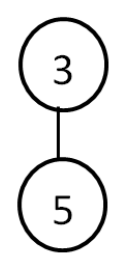

$\mathrm{H}_{2}$

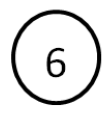

$\mathrm{H}_{3}$

Figura 9 - Exemplo de componentes, note que $G=H_{1} \cup H_{2} \cup H_{3}$.

Fonte: Elaborada pelo autor.

Como exemplo, na Figura $9 H_{1}$ é um clique de $G$ e $H_{3}$ é um conjunto independente de $G$, pois componentes também são considerados como subgrafos.

\section{2 Árvores}

Definição 2.2.1. Um grafo que não possui ciclos é chamado de floresta. Um grafo conectado que não possui ciclos é chamado de árvore. Uma folha é um vértice que possui grau 1.

Temos assim que uma floresta pode ser considerada como a reunião disjunta de uma ou mais árvores.

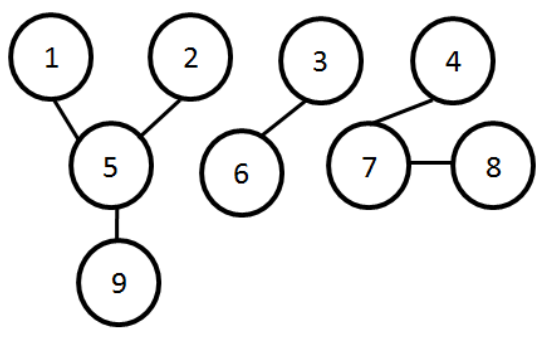

Floresta

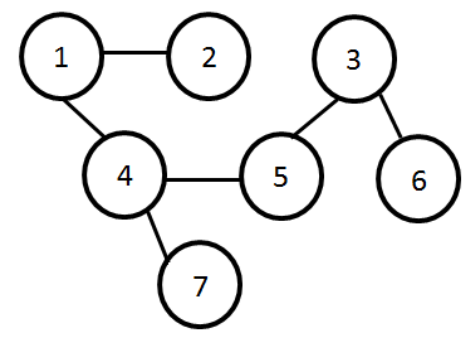

Árvore

Figura 10 - Exemplo de floresta e árvore.

Fonte: Elaborada pelo autor.

Definição 2.2.2. Uma aresta $e=(u, v) \in E(G)$ é uma ponte se a retirada de $(u, v)$ torna o grafo $G$ desconectado.

Note que toda aresta em uma árvore é uma ponte.

Teorema 2.2.1. Se $G$ é uma árvore com $n$ vértices, então $G$ possui $n-1$ arestas.

Demonstração. Provamos por indução em relação ao número de vértices em $G$. Se $n=1$, qualquer aresta em $G$ é da forma $(v, v)$, o que gera um ciclo em $G$. Como $G$ é uma 
árvore então não possui arestas, $\log$ possui $n-1=0$ arestas. Pela indução suponha que o teorema seja válido para árvores com no máximo $n-1$ vértices. Seja $G$ uma árvore com $n$ vértices e $(u, v) \in V(G)$. Como $G$ é uma árvore, $G \backslash\{(u, v)\}$ é desconectado, com duas componentes, cada uma sendo uma árvore com menos que $n$ vértices. Sejam $G_{1}$ e $G_{2}$ estes dois componentes com $n_{1}$ e $n_{2}$ vértices respectivamente. Temos que $n_{1}+n_{2}=n$. Como $n_{1}, n_{2}<n$, então pela hipótese de indução, $G_{1}$ tem $n_{1}-1$ arestas e $G_{2}$ tem $n_{2}-1$ arestas. Entretanto, $E(G)$ é a união disjunta de $E\left(G_{1}\right), E\left(G_{2}\right)$ e $(u, v), \log |E(G)|=$ $\left|E\left(G_{1}\right)\right|+\left|E\left(G_{2}\right)\right|+1=n_{1}-1+n_{2}-1+1=n-1$.

Vamos agora responder uma importante pergunta. Quantas árvores podemos formar com um número fixo $n$ de vértices? A resposta para esta pergunta se da pelo famoso Teorema de Cayley, formulado pelo matemático britânico Arthur Cayley (1821-1895). Para $n=3$ podemos verificar facilmente pela Figura 11 que a resposta é 3 .
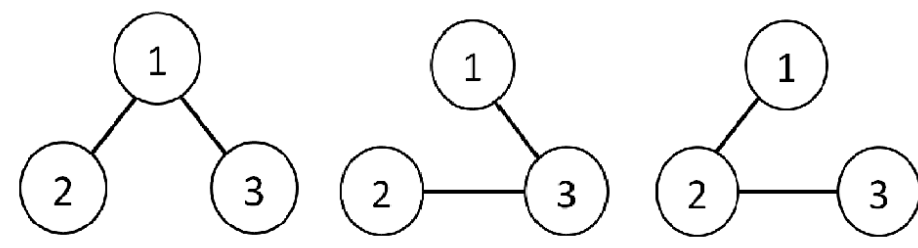

Figura 11 - As 3 possíveis árvores de 3 vértices.

Fonte: Elaborada pelo autor.

Teorema 2.2.2 (Teorema de Cayley). Existem $n^{n-2}$ formas diferentes de se formar uma árvore utilizando de um conjunto com $n$ vértices.

Para provar este teorema iremos utilizar de uma bijeção entre árvores com $V(G)=$ $B=\{1,2, \ldots, n\}$ e sequências em $B^{n-2}$, mas antes precisamos de alguns conceitos novos.

Um codificação para uma família de grafos é estipulada quando conseguímos obter uma relação biunívoca entre um grafo pertencente à família e um código que o represente. Quando é estabelecido uma codificação, podemos simplificar a geração aleatória e também a contagem de seus membros. É denominado codificação a obtenção do código a partir de um grafo, e decodificação a obtenção do grafo a partir de um código. Uma das codificações mais referenciadas na literatura (devido a prova do Teorema de Cayley e a propriedades referentes a árvores enraizadas) é a feita por Prüfer em 1918 para grafos da família árvores (PEREIRA; MARKENZON; VERNET, 2005).

Definição 2.2.3 (Código de Prüfer). Seja $T$ uma árvore em um conjunto ordenado $B$ de $n$ vértices. Obtemos a sequência de Prüfer (ou código de Prüfer) $f(T)$, deletando de forma iterativa as folhas com a menor "rótulo" (número $i$ que ordena os $v_{i}$ 's vértices) e anexamos o rótulo de seu vizinho a sequência. Após $n-2$ iterações vamos obter apenas uma única aresta e assim teremos produzido uma sequência $f(T)$ de comprimento $n-2$. 


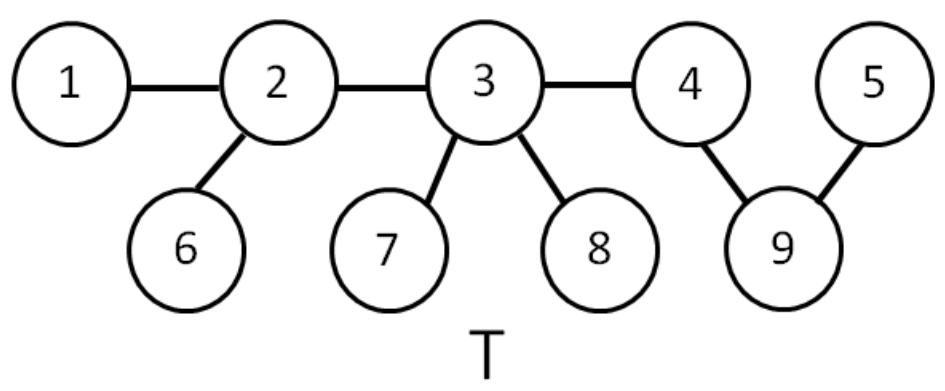

Figura 12 - Árvore $T$.

Fonte: Elaborada pelo autor.

Exemplo: para a árvore $T$ apresentado na Figura 12, obtemos a seguinte sequência de Prüfer.

- 2 (delete 1);

- 9 (delete 5);

- 2 (delete 6);

- 3 (delete 2);

- 3 (delete 7);

- 3 (delete 8);

- 4 (delete 3);

A aresta restante é a $(4,9)$, obtendo $f(T)=(2,9,2,3,3,3,4)$.

Proposiçao 2.2.3. Para um conjunto ordenado $B$ de $n$ elementos, o código de Prüfer $f$ é uma bijeção entre as possíveis árvores formadas com $B$ e as sequências em $B^{n-2}$.

Demonstração. Precisamos mostrar que cada sequência $\left(a_{1}, a_{2}, \ldots, a_{n-2}\right) \in B^{n-2}$ define uma única árvore $T$ tal que $f(T)=\left(a_{1}, a_{2}, \ldots, a_{n-2}\right)$. Se $n=2$, temos que existe apenas uma árvore que pode ser formada por estes dois vértices e o algoritmo ira informar uma sequência nula, a única sequência é a de comprimento zero. Assim, a alegação é satisfeita para $n=2$. Agora, assumimos $n \geq 2$, e que o teorema vale para todo conjunto de vértices ordenado $S^{\prime}$ de tamanho menor que $n$. Considere uma sequência $\left(a_{1}, a_{2}, \ldots, a_{n-2}\right) \in S^{n-2}$. Nós precisamo mostrar que $\left(a_{1}, a_{2}, \ldots, a_{n-2}\right)$ pode ser produzido exclusivamente pelo algoritmo.

Suponha que o algoritmo produza $f(T)=\left(a_{1}, a_{2}, \ldots, a_{n-2}\right)$ para alguma árvore $T$. Então os vértices $\left\{a_{1}, \ldots, a_{n-2}\right\}$ são precisamente aqueles que não são uma folha em $T$. Isto é verdade pois, se um vértice $v$ é uma folha em $T$, ele só pode aparecer em $f(T)$ se seu 
vizinho for excluído durante o algoritmo. Mas isso deixaria $v$ como um vértice isolado, o que é impossível. Por outro lado, se um vértice $v$ não é uma folha, temos que um de seus vizinhos deve ser excluído durante o algoritmo (ele não pode ser excluído antes que isso aconteça). Quando este vizinho de $v$ for excluído, v será adicionado ao código Prüfer por $T$, então estará em $\left\{a_{1}, a_{2}, \ldots, a_{n-2}\right\}$.

Isto implica que o rotulo da primeira folha removida de $T$ é o elemento mínimo do conjunto $S \backslash\left\{a_{1}, a_{2}, \ldots, a_{n-2}\right\}$. Seja $v$ este elemento. Em outras palavras, em cada árvore $T$ tal que $f(T)=\left(a_{1}, \ldots, a_{n-2}\right)$ o vértice $v$ é uma folha única cujo vizinho é $a_{1}$.

Por indução, existe uma única árvore $T^{\prime}$ com conjunto de vértices $S \backslash v$ tal que $f\left(T^{\prime}\right)=\left(a_{2}, \ldots, a_{n-2}\right)$. Adicionando o vértice $v$ e a aresta $\left(a_{1}, v\right)$ a $T^{\prime}$ iremos produzir uma única árvore $T \operatorname{com} f(T)=\left(a_{1}, a_{2}, . ., a_{n-2}\right)$.

Demonstração do Teorema de Cayley. Para provar o Teorema de Cayley, basta aplicarmos a Proposição 2.2.3 ao conjunto dos $n$ vértices em $B$. Note que existem $n^{n-2}$ sequências em $B^{n-2}$, provando o teorema.

\subsection{Grafos Aleatórios}

Grafos aleatórios são grafos criados a partir de processos aleatórios. Esta teoria se originou ao final dos anos de 1950 com os artigos On random graphs I e On the evolution of random graphs escritos por Paul Erdös e Alfréd Rényi (ERDöS; RéNYI, 1959),(ERDöS; RéNYI, 1960). O modelo original proposto por Erdös e Rényi é construído da seguinte forma. De inicio, é dado um conjunto de $n$ vértices em um tempo 0. Depois adicionamos $M$ arestas, uma de cada vez, de forma aleatória e uniforme entre os pares de vértices. Este modelo é identificado como modelo de grafo aleatório uniforme $G(n, M)$ e possui relação com outro modelo, chamado modelo binomial $G(n, p)$, no qual um conjunto de $n$ vértices é dado, e a cada passo de tempo, uma aresta é estabelecida independentemente entre dois vértices com probabilidade $p$. O modelo binomial foi introduzido por Gilbert (1959), e é conhecido que para uma determinada escolha de $M$ e $p$ os modelos são equivalentes. Um exemplo de construção do modelo binomial é apresentado na Figura 13.

Seja $Y_{E}$ a variável aleatória que representa a quantia de arestas em um grafo.

Proposiçao 2.3.1. Em um grafo aleatório binomial $G(n, p)$ a distribuição que representa a quantia de arestas do grafo é dada por

$$
\mathbb{P}\left(Y_{E}=M\right)=\left(\begin{array}{c}
\left(\begin{array}{c}
n \\
2
\end{array}\right) \\
M
\end{array}\right) p^{M}(1-p)^{\left(\begin{array}{c}
n \\
2
\end{array}\right)-M},
$$

para $M=0,1,2,3, \ldots$ 


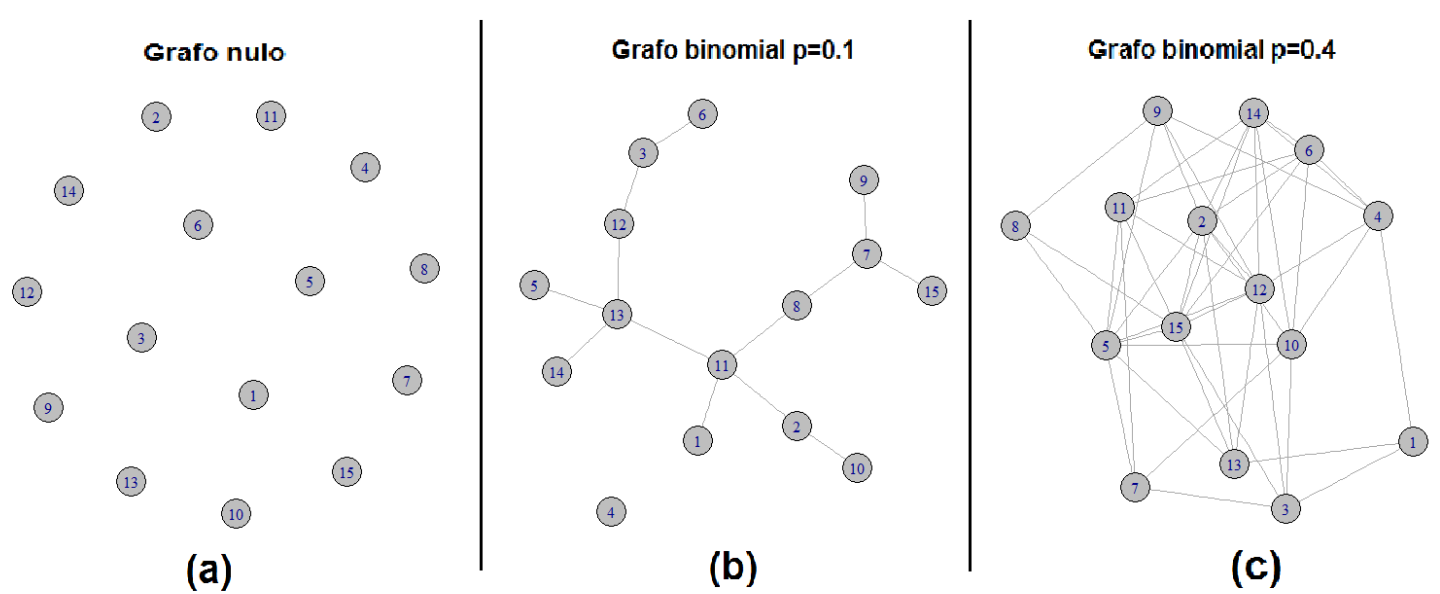

Figura 13 - (a)Grafo nulo com 15 vértices. (b) Realização do modelo binomial $G(15,0.1)$. (c) Realização do modelo binomial $G(15,0.4)$.

Fonte: Elaborada pelo autor.

Demonstração. No modelo $G(n, p)$ cada par de vértices tem chance de possuir uma aresta, isso nos dá que o máximo de arestas que o grafo aleatório pode ter é $\left(\begin{array}{l}n \\ 2\end{array}\right)=\frac{n(n-1)}{2}$. Para cada um destes possíveis pares a probabilidade de existir uma aresta é $p$, logo, a distribuição de existir uma aresta entre dois vértices é uma Bernoulli de parâmetro $p$. Como a existência de arestas é independente em relação ao par de vértices tomado, a probabilidade de existir $M$ arestas em $G(n, p)$ é dada por uma distribuição Binomial com parâmetros $\left(\begin{array}{l}n \\ 2\end{array}\right)$ e $p$.

Dado a proposição, temos que a média de arestas é dada por:

$$
\mathbb{E}\left[Y_{E}\right]=\sum_{M=0}^{\left(\begin{array}{l}
n \\
2
\end{array}\right)} M \mathbb{P}\left(Y_{E}=M\right)=p\left(\begin{array}{l}
n \\
2
\end{array}\right) .
$$

Teorema 2.3.2 (Média do grau). A esperança do grau de um vértice $v$ em um grafo aleatório $G(n, p)$ é dado por

$$
\mathbb{E}\left[D_{v}\right]=p(n-1),
$$

onde $D_{v}$ é uma variável aleatória que representa o grau de um vértice tomado ao acaso em $G(n, p)$.

Demonstração. Da mesma forma da prova anterior, podemos ver que no modelo binomial, um vértice esta conectado a outro vértice aleatório com probabilidade $p$. Este vértice possui $n-1$ possíveis outros vértices para se conectar. Logo, a distribuição de probabilidade de um vértice qualquer possuir grau $k$ é uma Binomial de parâmetros $n-1$ e $p$, ou seja,

$$
\mathbb{P}\left(D_{v}=k\right)=\left(\begin{array}{c}
n-1 \\
k
\end{array}\right) p^{k}(1-p)^{n-1-k} .
$$

Com isso, temos que a média de uma $\operatorname{Bin}(n-1, p)$ é $p(n-1)$, finalizando a demonstração. 
Várias outras propriedades já foram estabelecidas para o modelo binomial. Uma das mais importantes é sobre a probabilidade do limiar de conectividade do modelo, ou seja, a probabilidade de ele ser conectado dependendo da escolha de seus parâmetros (ver Definição 2.1.12). O teorema que descreve esta propriedade é apresentado a seguir:

Teorema 2.3.3 (Conectividade de $G(n, p))$. Considere o grafo aleatório binomial $G(n, p)$ com

$$
\lambda=p n ; \lambda>0
$$

Tem-se que:

1. Se $\lim _{n \rightarrow \infty} \lambda-\ln (n)=-\infty$, então

$$
\lim _{n \rightarrow \infty} \mathbb{P}[G(n, p) \text { ser conectado }]=0
$$

2. se $\lim _{n \rightarrow \infty} \lambda-\ln (n)=c$ uma constante, então

$$
\lim _{n \rightarrow \infty} \mathbb{P}[G(n, p) \text { ser conectado }]=e^{-e^{-c}} ;
$$

3. Se $\lim _{n \rightarrow \infty} \lambda-\ln (n)=\infty$, então

$$
\lim _{n \rightarrow \infty} \mathbb{P}[G(n, p) \text { ser conectado }]=1 .
$$

A demonstração do Teorema pode ser vista em Hofstad (2013, Cap 5. p. 104-107) ou em Bollobás (2001, Cap. 7). O propósito desta dissertação é provar um teorema análogo ao anterior, mas para um modelo criado a partir de aglomerações do grafo binomial. O modelo proposto, o teorema e a demonstração do mesmo serão apresentados no Capítulo 4. 


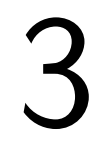

\section{MÉTODOS PROBABILÍSTICOS}

Neste capítulo serão apresentadas definições, conceitos e resultados que geralmente são utilizados como métodos probabilísticos. Tais métodos compõem um rico conjunto de informações que podem ser adotados para analisar propriedades de grafos aleatórios. Será dado maior ênfase nas distribuições Binomial e de Poisson, devido a relação que estas possuem com o modelo que vamos estudar. As informações aqui apresentadas foram baseadas em Durrett (2007), Ross (2010), Hofstad (2013) e James (2013).

\subsection{Convergência de variáveis aleatórias}

Em um grafo aleatório binomial vimos que o grau de um vértice é distribuído como uma variável aleatória $\operatorname{Bin}(n-1, p)$, ver Seção 2.3. Quando $n$ é grande e $p=\lambda / n$, sabemos que a $\operatorname{Bin}(n-1, p)$ se aproxima da variável aleatória de Poisson com média $\lambda$. Vamos verificar esta aproximação utilizando de métodos não tradicionais. Primeiramente, para formalizar que

$$
\operatorname{Bin}(n, p) \approx \operatorname{Poi}(n p)
$$

precisamos introduzir noções de convergência de variáveis aleatórias. Para isso, as variáveis aleatórias são definidas como funções em um espaço amostral.

Definição 3.1.1 (Convergência de variáveis aleatórias). Considere uma sequência de variáveis aleatórias $X, X_{1}, X_{2}, \ldots$

1. A sequência $\left\{X_{n}\right\}_{n \geq 1}$ converge em probabilidade para $X$ quando, para todo $\varepsilon>0$

$$
\lim _{n \rightarrow \infty} \mathbb{P}\left(\left|X_{n}-X\right|>\varepsilon\right)=0
$$

e denotamos por $X_{n} \stackrel{P}{\rightarrow} X$. 
2. A sequência $\left\{X_{n}\right\}_{n \geq 1}$ converge quase certamente para $X$ quando

$$
\mathbb{P}\left(\lim _{n \rightarrow \infty} X_{n}=X\right)=1
$$

e denotamos como $X_{n} \stackrel{a . s .}{\rightarrow} X$

3. A sequência $\left\{X_{n}\right\}_{n \geq 1}$ converge em distribuição para $X$ quando

$$
\lim _{n \rightarrow \infty} \mathbb{P}\left(X_{n} \leq x\right)=\mathbb{P}(X \leq x)
$$

para cada $x$ em que $F_{X}(x)=\mathbb{P}(X \leq x)$ é contínua. Denotamos como $X_{n} \stackrel{D}{\rightarrow} X$.

4. A sequência $\left\{X_{n}\right\}_{n \geq 1}$ converge para $X$ em $\mathcal{L}_{p}$ quando

$$
\lim _{n \rightarrow \infty} \mathbb{E}\left[\left|X_{n}-X\right|^{p}\right]=0
$$

denotamos como $X_{n} \stackrel{\mathcal{L}_{p}}{\rightarrow} X$. Quando $p=2$ dizemos que ocorre "convergência em média quadrática".

A convergência mais "forte" é a quase certa, devido ao fato que implica na convergência em probabilidade, que por sua vez implica na convergência em distribuição. As implicações inversas possuem restrições, caso $X_{n} \stackrel{P}{\rightarrow} X$ então existe uma subsequência de inteiros crescente $\left\{n_{k}\right\}_{k \geq 1}$ tal que $X_{n} k \stackrel{\text { a.s. }}{\rightarrow} X$, e a convergência em distribuição implica na convergência em probabilidade apenas se $X_{n} \stackrel{D}{\rightarrow} c$ com $c$ constante (JAMES, 2013, p. 195250). A Figura 14 resume as implicações de convergências.

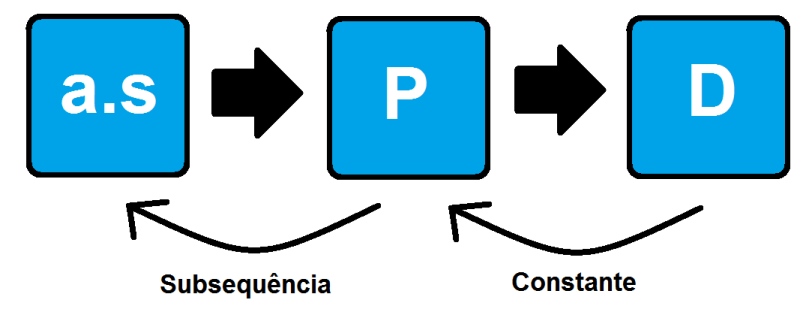

Figura 14 - Diagrama das convergências

Fonte: Elaborada pelo autor.

Convergências podem ser relacionadas com funções geradoras, ao qual são definidas a seguir.

Definição 3.1.2 (Funções geradoras). Seja $X$ uma variável aleatória.

1. A função característica de $X$ é dada como

$$
\phi_{X}(t):=\mathbb{E}\left[e^{i t X}\right] ; \quad t \in \mathbb{R}
$$


2. Se $X$ é discreta e não negativa. A função geradora de probabilidade (f.g.p) de $X$ é dada como

$$
G_{X}(t):=\mathbb{E}\left[t^{X}\right] ; \quad t \in[-1,1]
$$

3. A função geradora de momentos (f.g.m.) de $X$ é expressa como

$$
M_{X}(t):=\mathbb{E}\left[e^{t X}\right]
$$

para todo $t$ onde $\mathbb{E}\left[e^{t X}\right]$ seja finito.

Dadas as definições temos o primeiro teorema que as relaciona.

Teorema 3.1.1 (Critérios de convergência em distribuição). Uma sequência de variáveis aleatórias $\left\{X_{n}\right\}_{n=1}^{\infty}$ converge em distribuição para $X$

a) se, e só se, a função característica $\phi_{n}(t)$ de $X_{n}$ converge para a função característica $\phi_{X}(t)$ de $X$ para todo $t \in \mathbb{R}$.

b) quando, para algum $\varepsilon>0$, a função geradora de momentos $M_{n}(t)$ de $X_{n}$ converge para a função geradora de momentos $M_{X}(t)$ de $X$ para todo $|t|<\varepsilon$.

c) quando, para algum $\varepsilon>0$, a função geradora de probabilidade $G_{n}(t)$ de $X_{n}$ converge para $G_{X}(t)$ de $X$ para todo $|t|<1+\varepsilon$.

d) quando $X_{n}$ é não-negativo e de valores inteiros, e os momentos $\mathbb{E}\left[X_{n}^{r}\right]$ convergem para os momentos $\mathbb{E}\left[X^{r}\right]$ de $X$ para cada $r=1,2, \ldots$, desde que os momentos de $X$ satisfaçam

$$
\lim _{r \rightarrow \infty} \frac{\mathbb{E}\left[X^{r}\right] r^{m}}{r !}=0 \quad \forall m=0,1, \ldots
$$

As provas para os itens do teorema podem ser encontradas no capítulo 5 , seção 8 e 9 do livro Gut (2013).

Observação 3.1.2. Um exemplo de distribuição que satisfaz a Equação (3.8) é a distribuição de Poisson. Por uma expansão de Taylor da função geradora de momentos, se $M_{X}(t)<\infty \quad \forall t$, então

$$
M_{X}(t)=\mathbb{E}\left[e^{t X}\right]=\mathbb{E}\left[\sum_{r=0}^{\infty} \frac{(t X)^{r}}{r !}\right]=\sum_{r=0}^{\infty} \mathbb{E}\left[X^{r}\right] \frac{t^{r}}{r !},
$$

onde a passagem da média para dentro do somatório é satisfeita, devido a $X$ ser não negativo e a série ser convergente.

Como resultado, quando $M_{X}(t)<\infty$, temos pelo teste do n-ésimo termo que

$$
\lim _{r \rightarrow \infty} \mathbb{E}\left[X^{r}\right] \frac{t^{r}}{r !}=0
$$

Logo, quando $t>1$, a Expressão (3.8) é satisfeita. 
Basta então verificar que $M_{X}(t)<\infty \quad \forall t$ na distribuição de Poisson, o que ocorre pois

$$
M_{X}(t)=\mathbb{E}\left[e^{t X}\right]=\sum_{k=0}^{\infty} e^{t k} \frac{e^{-\lambda} \lambda^{k}}{k !}=e^{-\lambda} \sum_{k=0}^{\infty} \frac{\left(\lambda e^{t}\right)^{k}}{k !}=e^{-\lambda} e^{\lambda e^{t}}=e^{\lambda\left(e^{t}-1\right)}<\infty \quad \forall t \in \mathbb{R} .
$$

Assim, a distribuição de Poisson satisfaz a Equação (3.8).

Definição 3.1.3 (Momentos Fatoriais). Seja $X$ uma variável aleatória. Escrevemos

$$
(X)_{m}:=X(X-1) \ldots(X-m+1)
$$

e denominamos $\mathbb{E}\left[(X)_{m}\right]$ como sendo o m-ésimo momento fatorial de $X$.

Observação 3.1.3 (Momentos fatoriais de uma variável aleatória de Poisson). Os momentos fatoriais de uma variável aleatória de Poisson com média $\lambda$ são dados por

$$
\begin{aligned}
\mathbb{E}\left[(X)_{m}\right]= & \mathbb{E}[X(X-1) \ldots(X-m+1)] \\
= & \sum_{x=0}^{\infty} x(x-1) \ldots(x-m+1) \mathbb{P}(X=x) \\
= & \sum_{x=m}^{\infty} x(x-1) \ldots(x-m+1) \frac{e^{-\lambda} \lambda^{x}}{x !} \\
& \text { pois para } x=0, x=1, \ldots, x=r-1 \text { a expressão resulta em } 0, \\
= & \lambda^{m} \sum_{x=m}^{\infty} e^{-\lambda} \frac{\lambda^{x-m}}{(x-m) !}=\lambda^{m} e^{-\lambda} \sum_{x-m=0}^{\infty} \frac{\lambda^{x-m}}{(x-m) !} \\
= & \lambda^{m} e^{-\lambda} e^{\lambda}=\lambda^{m} .
\end{aligned}
$$

Para complementar, temos que a expressão dos momentos $\mathbb{E}\left[X^{m}\right]$ para $X \sim \operatorname{Poi}(\lambda)$ é dada por

$$
\begin{aligned}
\mathbb{E}\left[X^{m}\right] & =\sum_{k=0}^{\infty} k^{m} e^{-\lambda} \frac{\lambda^{k}}{k !} \\
& =\sum_{k=1}^{\infty} k^{m} e^{-\lambda} \frac{\lambda^{k}}{k !} \\
& =\lambda \sum_{k=1}^{\infty} k^{m-1} e^{-\lambda} \frac{\lambda^{k-1}}{(k-1) !} \\
& =\lambda \sum_{l=0}^{\infty}(l+1)^{m-1} e^{-\lambda} \frac{\lambda^{l}}{(l) !} \\
& =\lambda \mathbb{E}\left[(X+1)^{m-1}\right] .
\end{aligned}
$$

Note que a expressão de $\mathbb{E}\left[X^{m}\right]$ parece mais complicada de se trabalhar do que a expressão para $\mathbb{E}\left[(X)_{m}\right]$. Assim, gostaríamos de criar um outro item para a convergência em distribuição, semelhante ao item $d$ ) do Teorema 3.1.1 mas utilizando agora de $\mathbb{E}\left[(X)_{m}\right]$. 
Vamos verificar que para uma variável aleatória $X$ que toma valores $\{0,1, \ldots, n\}$, os momentos fatoriais de $X$ determinam unicamente a função de probabilidade. Para isso tomamos

$$
I_{\{X=k\}}=\left(\begin{array}{l}
X \\
k
\end{array}\right)(1-1)^{X-k},
$$

onde $I_{\{X=k\}}$ denota uma variável aleatória indicadora, e utilizamos da convenção de que $0^{0}=1$.

O Binômio de Newton nos diz que, para $a, b$ constantes e $n \in \mathbb{N}$

$$
(a+b)^{n}=\sum_{i=0}^{n}\left(\begin{array}{c}
n \\
i
\end{array}\right) a^{n-i} b^{i}
$$

Assim, temos que

$$
I_{\{X=k\}}=\left(\begin{array}{c}
X \\
k
\end{array}\right) \sum_{i=0}^{X-k}(-1)^{i}\left(\begin{array}{c}
X-k \\
i
\end{array}\right)=\sum_{i=0}^{n}(-1)^{i}\left(\begin{array}{c}
X \\
k
\end{array}\right)\left(\begin{array}{c}
X-k \\
i
\end{array}\right),
$$

onde por convenção, usamos que $\left(\begin{array}{l}n \\ k\end{array}\right)=0$ quando $k<0$ ou $k>n$.

Redefinindo os binomiais e usando $m=k+i$, teremos

$$
\begin{aligned}
I_{\{X=k\}} & =\sum_{i=0}^{n}(-1)^{i} \frac{X !}{(X-k) ! k !} \frac{(X-k) !}{(X-k-i) ! i !} \\
& =\sum_{m-k=0}^{n}(-1)^{m-k} \frac{X !}{(X-k) ! k !} \frac{(X-k) !}{(X-k-m+k) !(m-k) !} \\
& =\sum_{m=k}^{n}(-1)^{k+m} \frac{X !}{(X-m) !} \frac{1}{(m-k) ! k !} \\
& =\sum_{m=k}^{n}(-1)^{k+m} \frac{(X)_{m}}{(m-k) ! k !} .
\end{aligned}
$$

Aplicando a esperança ficamos com

$$
\mathbb{P}(X=k)=\sum_{m=k}^{n}(-1)^{k+m} \frac{\mathbb{E}\left[(X)_{m}\right]}{(m-k) ! k !}
$$

sendo uma expressão que define a função de probabilidade de $X$ em função dos momentos fatoriais, como queríamos demonstrar.

Podemos encontrar resultados similares para variáveis aleatória ilimitadas, com a diferença que

$$
\mathbb{P}(X=k)=\sum_{m=k}^{\infty}(-1)^{m+r} \frac{\mathbb{E}\left[(X)_{m}\right]}{(m-k) ! k !}
$$

e que

$$
\sum_{m=k}^{n}(-1)^{m+k} \frac{\mathbb{E}\left[(X)_{m}\right]}{(m-k) ! k !}
$$


é alternadamente menor que $\mathbb{P}(X=k)$ se $n=s$ e $s+k$ for ímpar, e maior que $\mathbb{P}(X=k)$ se $n=t$ e $t+k$ for par.

Note que, se

$$
\lim _{n \rightarrow \infty} \sum_{m \geq n} \frac{\mathbb{E}\left[(X)_{m}\right]}{(m-k) !}=0
$$

então

$$
\lim _{n \rightarrow \infty}\left|\sum_{m \geq n}(-1)^{k+m} \frac{\mathbb{E}\left[(X)_{m}\right]}{(m-k) ! k !}\right| \leq \lim _{n \rightarrow \infty} \sum_{m \geq n} \frac{\mathbb{E}\left[(X)_{m}\right]}{(m-k) ! k !}=0,
$$

e assim

$$
\mathbb{P}(X=k)=\sum_{m=k}^{\infty}(-1)^{k+m} \frac{\mathbb{E}\left[(X)_{m}\right]}{(m-k) ! k !} \approx \sum_{m=k}^{n}(-1)^{k+m} \frac{\mathbb{E}\left[(X)_{m}\right]}{(m-k) ! k !},
$$

para um $n$ suficientemente grande. As informações anteriores implicam no seguinte teorema:

Teorema 3.1.4 (Convergência da v.a. de Poisson). Um sequência de variáveis aleatórias $\left\{X_{n}\right\}_{n \geq 1}$, que toma valores inteiros, converge em distribuição para uma Poisson com parâmetro $\lambda$ quando, $\forall m=1,2, \ldots$

$$
\lim _{n \rightarrow \infty} \mathbb{E}\left[\left(X_{n}\right)_{m}\right]=\lambda^{m}
$$

Demonstração. Por hipótese $\lim _{n \rightarrow \infty} \mathbb{E}\left[\left(X_{n}\right)_{m}\right]=\lambda^{m}, \forall m=1,2, \ldots$. Seja $X$ uma variável aleatório com distribuição de Poisson com média $\lambda$, temos por (3.9) que $\mathbb{E}\left[(X)_{m}\right]=\lambda^{m}$, $\forall m=1,2, \ldots$ Logo, $\lim _{n \rightarrow \infty} \mathbb{E}\left[\left(X_{n}\right)_{m}\right]=\mathbb{E}\left[(X)_{m}\right]$. Como vimos anteriormente que os momentos fatoriais definem unicamente a função de probabilidade de uma variável aleatória, se tomarmos um $n$ suficientemente grande, teremos que $\mathbb{P}\left(X_{n}=k\right)=\mathbb{P}(X=k)$. E assim, usando as definições de convergência, concluímos que $X_{n} \stackrel{D}{\rightarrow} X$.

O teorema anterior é particularmente conveniente quando lidamos com uma soma de indicadoras, ou seja, quando

$$
X_{n}=\sum_{i \in \mathcal{J}_{n}} I_{i, n}
$$

onde $I_{i, n}$ é uma variável aleatória que assume valores 0 ou 1 . Nesta ocasião, temos o seguinte resultado:

Teorema 3.1.5 (Momentos fatoriais de somas de indicadoras). Seja $X=\sum_{i \in \mathcal{J}} I_{i}$ uma soma de indicadoras, temos que

$$
\mathbb{E}\left[(X)_{m}\right]=\sum_{i_{1}, \ldots, i_{m} \in \mathcal{J}}^{*} \mathbb{E}\left[\prod_{l=1}^{m} I_{i l}\right]=\sum_{i_{1}, \ldots, i_{m} \in \mathcal{J}}^{*} \mathbb{P}\left(I_{i 1}=I_{i 2}=\ldots=I_{i m}=1\right),
$$

onde $\sum_{i_{1}, \ldots, i_{m} \in \mathcal{J}}^{*}$ denota uma soma sobre índices distintos. 
Demonstração. Vamos utilizar a prova por indução sobre $m \geq 1$. Para $m=1$ nós temos que $(X)_{1}=X$, e assim (3.10) segue do fato de que a esperança da soma de variáveis aleatórias indicadoras é a soma das esperanças. Note que é suficiente provar apenas para as indicadoras $I_{i}$ no qual $\mathbb{P}\left(I_{i}=1\right)>0$. Assim, para $m \geq 2$, escrevemos

$$
\mathbb{E}\left[(X)_{m}\right]=\sum_{I_{1} \in \mathcal{J}} \mathbb{E}\left[I_{i 1}(X-1) \ldots(X-m+1)\right]
$$

Denotamos por $\mathbb{P}_{i 1}$ a distribuição condicional dada por $I_{i 1}=1$, isto é, para qualquer evento $E$, nós temos

$$
\left.\mathbb{P}_{i 1}(E):=\mathbb{P}\left(E \mid\left\{I_{i 1}=1\right)\right\}\right)=\frac{\mathbb{P}\left(E \cap\left\{I_{i 1}=1\right)\right\}}{\mathbb{P}\left(I_{i 1}=1\right)} .
$$

E portanto denotamos

$$
\mathbb{E}_{i 1}[X]:=\mathbb{E}\left[X \mid I_{i 1}=1\right]
$$

Assim, podemos reescrever

$$
\mathbb{E}\left[I_{i 1}(X-1) \ldots(X-m+1)\right]=\mathbb{P}\left(I_{i 1}=1\right) \mathbb{E}_{i 1}[(X-1) \ldots(X-m+1)] .
$$

Definimos agora

$$
Y=X-I_{i 1}=\sum_{j \in \mathcal{J} \backslash\{i 1\}} I_{j} .
$$

Note que, condicionando $I_{i 1}=1$, nós temos $X=Y+1$. Como resultado, obtemos

$$
\mathbb{E}_{i 1}[(X-1) \ldots(X-m+1)]=\mathbb{E}_{i 1}[Y \ldots(Y-m+2)]=\mathbb{E}_{i 1}\left[(Y)_{m-1}\right]
$$

Aplicando a hipótese de indução para $\mathbb{E}_{i 1}\left[(Y)_{m-1}\right]$, obtemos

$$
\mathbb{E}_{i 1}\left[(Y)_{m-1}\right]=\sum_{i 2, \ldots, i m \in \mathcal{J} \backslash\{i 1\}}^{*} \mathbb{P}_{i 1}\left(I_{i 2}=\ldots=I_{i m}=1\right) .
$$

Como resultado, chegamos a

$$
\mathbb{E}\left[(X)_{m}\right]=\sum_{i 1 \in \mathcal{J}} \mathbb{P}\left(I_{i 1}=1\right) \sum_{i 2, \ldots, i m \in \mathcal{J} \backslash\{i 1\}}^{*} \mathbb{P}_{i 1}\left(I_{i 2}=\ldots=I_{i m}=1\right),
$$

e completamos a prova, observando que

$$
\mathbb{P}\left(I_{i 1}=1\right) \mathbb{P}_{i 1}\left(I_{i 2}=\ldots=I_{i m}=1\right)=\mathbb{P}\left(I_{i 1}=I_{i 2}=\ldots=I_{i m}=1\right),
$$

e que

$$
\sum_{i 1 \in \mathcal{J}} \sum_{i 2, \ldots, i m \in \mathcal{J} \backslash\{i 1\}}^{*}=\sum_{i 1, i 2, \ldots, i m \in \mathcal{J}}^{*}
$$


Verificamos agora, que uma distribuição binomial de parâmetros $n$ e $p=\lambda / n$ converge em distribuição para uma variável aleatória de Poisson com parâmetro $\lambda$. Devido a todas as informações apresentadas até o momento, basta que calculemos os momentos fatoriais destas duas distribuições para verificar a convergência. Para a distribuição de Poisson, já sabemos por cálculos anteriores em (3.9) que $\mathbb{E}\left[(X)_{m}\right]=\lambda^{m}$. Agora, seja $X_{n}$ uma variável aleatória $\operatorname{Bin}(n, p)$ onde $p=\lambda / n$. Então, temos que

$$
\begin{aligned}
\mathbb{E}\left[\left(X_{n}\right)_{m}\right] & =\mathbb{E}\left[X_{n}\left(X_{n}-1\right) \ldots\left(X_{n}-m+1\right)\right] \\
& =\sum_{i 1, \ldots, i m \in \mathcal{J}} \mathbb{P}\left(I_{i 1}=I_{i 2}=\ldots=I_{i m}=1\right)
\end{aligned}
$$

Como, para todo $j=1,2, \ldots, m, \mathbb{P}\left(I_{i j}\right)=\lambda / n$, e a quantia de vezes que somamos é um arranjo de $m$ elementos dos $n$ possíveis, temos que

$$
\begin{aligned}
\mathbb{E}\left[\left(X_{n}\right)_{m}\right] & =n(n-1) \ldots(n-m+1) \frac{\lambda^{m}}{n^{m}} \\
& =1\left(1-\frac{1}{n}\right)\left(1-\frac{2}{n}\right)\left(1-\frac{3}{n}\right) \ldots\left(1-\frac{(m-1)}{n}\right) \lambda^{m}
\end{aligned}
$$

Portanto, $\lim _{n \rightarrow \infty} \mathbb{E}\left[\left(X_{n}\right)_{m}\right]=\lambda^{m}$ e pelos Teoremas 3.1.4 e 3.1.5, comprovamos a convergência em distribuição.

Existem também versões dos teoremas anteriores para variáveis multidimensionais, para mais informações ver Hofstad (2013, p. 29-30).

\subsection{Acoplamento e Método de Stein}

Neste capítulo vamos mostrar um pouco das aplicações do método de acoplamento e utilizá-lo para verificar, a partir de uma distância, a convergência em probabilidade entre duas variáveis aleatórias.

Definição 3.2.1 (Acoplamento de variáveis aleatórias). As variáveis aleatórias $\left(\widehat{X}_{1}, \widehat{X}_{2}, \ldots\right.$, $\left.\widehat{X}_{n}\right)$ são um acoplamento das variáveis aleatórias $X_{1}, X_{2}, \ldots, X_{n}$, quando $\left(\widehat{X}_{1}, \widehat{X}_{2}, \ldots, \widehat{X}_{n}\right)$ são definidas no mesmo espaço de probabilidade, e são tais que as distribuições marginais de $\widehat{X}_{i}$, são as mesmas que as distribuições marginais de $X_{i}$ para todo $i=1, \ldots, n$. Podemos escrever com isso que, para todo subconjunto mensurável $E$ de $\mathbb{R}$, nós temos

$$
\mathbb{P}\left(\widehat{X}_{i} \in E\right)=\mathbb{P}\left(X_{i} \in E\right) \text { para } i=1,2, \ldots, n
$$

Note que pela definição existem várias formas de estabelecer um acoplamento, uma delas poderia ser tomando $\left(\widehat{X}_{1}, \ldots, \widehat{X}_{n}\right)$ independentes, com $\widehat{X}_{i}$ tendo a mesma distribuição de $X_{i}$ para $i=1,2, \ldots, n$. Por exemplo, podemos definir um acoplamento para duas variáveis 
$X \sim \operatorname{Ber}(p)$ e $Y \sim \operatorname{Ber}(q)$ tomando o vetor de probabilidade $(\widehat{X}, \widehat{Y})$ como

$$
\begin{aligned}
\mathbb{P}(\widehat{X}=\widehat{Y}=0) & =(1-p)(1-q), \\
\mathbb{P}(\widehat{X}=\widehat{Y}=1) & =p q, \\
\mathbb{P}(\widehat{X}=1, \widehat{Y}=0) & =p(1-q), \\
\mathbb{P}(\widehat{X}=0, \widehat{Y}=1) & =(1-p) q .
\end{aligned}
$$

Veja que apenas tomamos a distribuição conjunta de $(X, Y) \operatorname{com} X$ e $Y$ independentes.

A definição a seguir nos ajuda a utilizar de um acoplamento mais específico e também é usado na prova do teorema de conectividade principal da dissertação.

Definição 3.2.2 (Distância de variação total). Dadas duas distribuições de probabilidades $\mu$ e $\boldsymbol{v}$ sobre um espaço de estados finito $\Omega$, definimos a distância de variação total entre $\mu$ e $v$ como

$$
d_{T V}(\mu, v)=\max _{A \subseteq \Omega}|\mu(A)-v(A)|,
$$

onde $\mu(A)$ e $\boldsymbol{v}(A)$ são as probabilidades do evento $A$ sobre as medidas $\mu$ e $v$. Quando $\mu$ e $v$ tem funções de probabilidade discretas $p=\left\{p_{x}\right\}_{x \in X}$ e $q=\left\{q_{x}\right\}_{x \in X}$ temos então que para cada medida $A \subseteq \mathbb{R}$

$$
\mu(A)=\sum_{x \in A} p_{x}, \quad v(A)=\sum_{x \in A} q_{x}
$$

Dada a definição, vamos verificar que para duas funções de probabilidade $p$ e $q$,

$$
d_{T V}(p, q)=\frac{1}{2} \sum_{x \in \Omega}\left|p_{x}-q_{x}\right| .
$$

Para isso, seja $\Omega_{1}=\left\{x \in \Omega ; p_{x} \geq q_{x}\right\}$ e $\Omega_{2}=\left\{x \in \Omega ; p_{x}<q_{x}\right\}$. Uma vez que $p_{x}$ e $q_{x}$ são funções de densidade de probabilidade, a soma $\sum_{x \in \Omega}\left(p_{x}-q_{x}\right)$ é igual a zero. Logo,

$$
\begin{aligned}
\sum_{x \in \Omega}\left|p_{x}-q_{x}\right| & =\sum_{x \in \Omega_{1}}\left(p_{x}-q_{x}\right)-\sum_{x \in \Omega_{2}}\left(p_{x}-q_{x}\right), \\
0=\sum_{x \in \Omega}\left(p_{x}-q_{x}\right) & =\sum_{x \in \Omega_{1}}\left(p_{x}-q_{x}\right)+\sum_{x \in \Omega_{2}}\left(p_{x}-q_{x}\right) .
\end{aligned}
$$

Adicionando ou subtraindo as equações acima, obtemos

$$
\sum_{x \in \Omega}\left|p_{x}-q_{x}\right|=2 \sum_{x \in \Omega_{1}}\left(p_{x}-q_{x}\right)=-2 \sum_{x \in \Omega_{2}}\left(p_{x}-q_{x}\right)
$$

Agora, consideramos $A$ sendo qualquer subconjunto de $\Omega$. Repartimos o conjunto A como

$$
A_{1}=A \cap \Omega_{1} \quad \text { e } \quad A_{2}=A \cap \Omega_{2}
$$


temos então que, para duas distribuições discretas $\mu$ e $v$,

$$
\begin{aligned}
|\mu(A)-v(A)| & =\left|\sum_{x \in A_{1}} p_{x}+\sum_{x \in A_{2}} p_{x}-\sum_{x \in A_{1}} q_{x}-\sum_{x \in A_{2}} q_{x}\right| \\
& =\left|\sum_{x \in A_{1}}\left(p_{x}-q_{x}\right)+\sum_{x \in A_{2}}\left(p_{x}-q_{x}\right)\right| \\
& =|\alpha A+\beta A|,
\end{aligned}
$$

onde $\alpha A$ resulta em um valor não-negativo e $\beta A$ em um valor não-positivo. Logo, quando houver soma destes dois elementos, o valor resultante será menor ou igual ao valor de apenas um deles. Assim, vemos que o valor máximo para qualquer conjunto $A$ será $|\alpha A|$ se $A=\Omega_{1}$ ou $|\beta A|$ se $A=\Omega_{2}$. E portanto, para qualquer subconjunto $A$ possível, temos que

$$
d_{T V}(p, q)=\max _{A \subseteq \Omega}|\mu(A)-v(A)|=\sum_{x \in \Omega_{1}}\left(p_{x}-q_{x}\right)=\left|\sum_{x \in \Omega_{2}}\left(p_{x}-q_{x}\right)\right|=\frac{1}{2} \sum_{x \in \Omega}\left|p_{x}-q_{x}\right|,
$$

o que queríamos verificar.

De forma semelhante quando $\mu$ e $\boldsymbol{v}$ forem funções de distribuição correspondentes a duas densidades contínuas $f=\{f(x)\}_{x \in \mathbb{R}}$ e $g=\{g(x)\}_{x \in \mathbb{R}}$, temos para cada $A \subseteq \mathbb{R}$ mensurável,

$$
\mu(A)=\int_{A} f(x) d x, \quad v(A)=\int_{A} g(x) d x
$$

e assim obtemos

$$
d_{T V}(f, g)=\frac{1}{2} \int_{-\infty}^{\infty}|f(x)-g(x)| d x
$$

A seguir temos um importante teorema que relaciona o acoplamento com a distância de variação total, e que nos ajudará a estabelecer convergência entre duas variáveis aleatórias discretas.

Sejam $X$ e $Y$ duas variáveis aleatórias discretas com

$$
\mathbb{P}(X=x)=p_{x}, \quad \mathbb{P}(Y=y)=q_{y}, \quad x \in X, y \in \mathcal{Y}
$$

onde $\left\{p_{x}\right\}_{x \in X}$ e $\left\{q_{y}\right\}_{y \in y}$ são quaisquer duas funções de probabilidade em dois subconjuntos $x$ e $y$ do mesmo espaço.

Teorema 3.2.1 (Teorema de Strassen). Para quaisquer duas variáveis aleatórias discretas $X$ e $Y$, existe um acoplamento $(\widehat{X}, \widehat{Y})$ de $X$ e $Y$ tal que

$$
\mathbb{P}(\widehat{X} \neq \widehat{Y})=d_{T V}(p, q)
$$

e para qualquer acoplamento $\left(\widehat{X}^{\prime}, \widehat{Y}^{\prime}\right)$ de $X$ e $Y$,

$$
\mathbb{P}\left(\widehat{X}^{\prime} \neq \widehat{Y}^{\prime}\right) \geq d_{T V}(p, q)
$$


Demonstração. Inicialmente, definimos o vetor aleatório $(\widehat{X}, \widehat{Y})$ por

$$
\begin{gathered}
\mathbb{P}(\widehat{X}=\widehat{Y}=x)=\min \left\{p_{x}, q_{x}\right\}, \\
\mathbb{P}(\widehat{X}=x, \widehat{Y}=y)=\frac{\left(p_{x}-\min \left\{p_{x}, q_{x}\right\}\right)\left(q_{y}-\min \left\{p_{y}, q_{y}\right\}\right)}{\frac{1}{2} \sum_{z}\left|p_{z}-q_{z}\right|}, x \neq y .
\end{gathered}
$$

Veja que

$$
\sum_{x}\left|p_{x}-q_{x}\right|=\sum_{x}\left(p_{x}-q_{x}\right) I_{\left\{p_{x} \geq q_{x}\right\}}-\sum_{x}\left(p_{x}-q_{x}\right) I_{\left\{p_{x}<q_{x}\right\}},
$$

e também que

$$
0=1-1=\sum_{x}\left(p_{x}-q_{x}\right)=\sum_{x}\left(p_{x}-q_{x}\right) I_{\left\{p_{x} \geq q_{x}\right\}}+\sum_{x}\left(p_{x}-q_{x}\right) I_{\left\{p_{x}<q_{x}\right\}},
$$

onde $I_{\{.\}}$é uma função indicadora.

Adicionando as duas igualdades anteriores obtemos

$$
\sum_{x}\left|p_{x}-q_{x}\right|=2 \sum_{x}\left(p_{x}-q_{x}\right) I_{\left\{p_{x} \geq q_{x}\right\}}
$$

Mas, observe que

$$
\sum_{x}\left(p_{x}-\min \left(p_{x}, q_{x}\right)\right)=\sum_{x}\left(p_{x}-q_{x}\right) I_{\left\{p_{x} \geq q_{x}\right\}},
$$

e assim, temos que

$$
\sum_{x}\left(p_{x}-\min \left\{p_{x}, q_{x}\right\}\right)=\sum_{x}\left(q_{x}-\min \left\{p_{x}, q_{x}\right\}\right)=\frac{1}{2} \sum_{x}\left|p_{x}-q_{x}\right|,
$$

fazendo com que o vetor aleatório definido anteriormente por (3.13) e (3.14) seja uma distribuição de probabilidade.

Por construção,

$$
\mathbb{P}(\widehat{X}=x)=p_{x}, \quad \mathbb{P}(\widehat{Y}=y)=q_{y},
$$

de modo que $\widehat{X}$ e $\widehat{Y}$ possuem distribuição marginal de acordo com a Definição 3.2.1. Entretanto temos por (3.14) que

$$
\begin{aligned}
\mathbb{P}(\widehat{X} \neq \widehat{Y}) & =\sum_{x, y} \frac{\left(p_{x}-\min \left\{p_{x}, q_{x}\right\}\right)\left(q_{y}-\min \left\{p_{y}, q_{y}\right\}\right)}{\frac{1}{2} \sum_{z}\left|p_{z}-q_{z}\right|} \\
& =\frac{1}{2} \sum_{x}\left|p_{x}-q_{x}\right|=d_{T V}(p, q) .
\end{aligned}
$$

Logo, podemos sempre definir um acoplamento que resulte em (3.11). 
Agora veja que, para todo $x$,

$$
\mathbb{P}\left(\widehat{X}^{\prime}=\widehat{Y}^{\prime}=x\right) \leq \mathbb{P}\left(\widehat{X}^{\prime}=x\right)=\mathbb{P}(X=x)=p_{x}
$$

e também

$$
\mathbb{P}\left(\widehat{X}^{\prime}=\widehat{Y}^{\prime}=x\right) \leq \mathbb{P}\left(\widehat{Y}^{\prime}=x\right)=\mathbb{P}(Y=x)=q_{x}
$$

Logo, para qualquer acoplamento, nós devemos ter

$$
\mathbb{P}\left(\widehat{X}^{\prime}=\widehat{Y}^{\prime}=x\right) \leq \min \left(p_{x}, q_{x}\right)
$$

Porém, qualquer acoplamento deve ser tal que

$$
\mathbb{P}\left(\widehat{X}^{\prime}=\widehat{Y}^{\prime}\right)=\sum_{x} \mathbb{P}\left(\widehat{X}^{\prime}=\widehat{Y}^{\prime}=x\right) \leq \sum_{x} \min \left(p_{x}, q_{x}\right)
$$

e como resultado temos

$$
\mathbb{P}\left(\widehat{X}^{\prime} \neq \widehat{Y}^{\prime}\right) \geq 1-\sum_{x} \min \left(p_{x}, q_{x}\right)=\frac{1}{2} \sum_{x}\left|p_{x}-q_{x}\right|,
$$

provando a Equação (3.12).

Analisando a definição de acoplamento e o resultado do teorema anterior, se tivermos $\lim _{n \rightarrow \infty} \mathbb{P}\left(\widehat{X_{n}} \neq \widehat{Y}\right)=0$, onde $\left\{X_{n}\right\}$ é uma sequência de variáveis aleatórias i.i.d., teremos que $\lim _{n \rightarrow \infty} \mathbb{P}\left(X_{n} \neq Y\right)=0$ pela igualdade das funções marginais, o que ocasiona em uma convergência em probabilidade, pois significaria que $\lim _{n \rightarrow \infty} \mathbb{P}\left(\left|X_{N}-Y\right|>\varepsilon\right)=0$, para todo $\varepsilon>0$.

Vamos apresentar agora um importante lema utilizado para provar convergência na demonstração do principal teorema de conectividade para o modelo grafo aleatório estudado na dissertação. Para isso precisamos definir o conceito de variáveis positivas relacionadas.

Definição 3.2.3. As variáveis aleatórias $\left\{I_{z}\right\}_{z}$ (v.a. indicadoras) são ditas positivamente relacionadas se elas satisfazem as seguintes condições:

- Para cada $z$ existe uma família de variáveis aleatórias $\left\{J_{l}^{z}\right\}, l \neq z$, tal que a distribuição conjunta de $\left\{J_{l}^{z}\right\}_{l}$ seja a mesma que a distribuição condicional de $\left\{I_{l}\right\}_{l}$ dado $I_{z}=1$

- $J_{l}^{z} \geq I_{l}$ para todo $l \neq z$.

Categoricamente, podemos usar $\mathcal{L}\left(\left\{J_{l}^{z}\right\}_{l}\right)=\mathcal{L}\left(\left\{I_{l}\right\}_{l} \mid I_{z}=1\right)$, e $J_{l}^{z} \geq I_{l}$ para todo $l \neq z$, onde $\mathcal{L}\left(\left\{Y_{l}\right\}_{l}\right)$ denota a distribuição conjunta das variáveis aleatórias $\left\{Y_{l}\right\}_{l}$. 
Lema 1 (Método de Stein). Dadas as variáveis aleatórias $\left\{I_{z}\right\}_{z}$, seja $X:=\sum_{z} I_{z}$. Se $\left\{I_{z}\right\}_{z}$ são positivas relacionadas, então

$$
d_{T V}(X, \operatorname{Po}(\lambda)) \leq \frac{V(X)}{\mathbb{E}[X]}-1+2 \max _{1 \leq z \leq N}\left\{\mathbb{E}\left(I_{z}\right)\right\},
$$

onde $\lambda=\mathbb{E}[X]$ e $d_{T V}($, ) é a distância de variação total.

A demonstração deste teorema é encontrada em Janson, Luczak e Rucinski (2000).

\subsection{Desigualdades probabilísticas}

Apresentamos aqui algumas das principais desigualdades, que são utilizadas frequentemente em demonstrações envolvendo estocasticidade. Estes resultados são ferramentas poderosas e serão utilizados no desenvolvimento da demonstração do teorema principal, que envolve a conectividade do grafo no modelo proposto para análise.

Teorema 3.3.1 (Desigualdade de Markov). Seja $X$ uma variável aleatória não negativa com $\mathbb{E}[X]<\infty$. Temos que, para $a>0$,

$$
\mathbb{P}(X \geq a) \leq \frac{\mathbb{E}[X]}{a}
$$

Demonstração. Definimos para $a>0$,

$$
I= \begin{cases}1 & \text { se } X \geq a \\ 0 & \text { caso contrário }\end{cases}
$$

Como $X \geq 0$ temos que $I \leq X / a$. Aplicando a esperança temos

$$
\mathbb{P}(X \geq a)=\mathbb{E}[I] \leq \frac{\mathbb{E}[X]}{a},
$$

concluindo a prova.

Corolário 3.3.2 (Método do primeiro momento). Seja $X$ uma variável aleatória não negativa que toma valores inteiros e $\mathbb{E}[X] \leq m$, então

$$
\mathbb{P}(X=0) \geq 1-m \text {. }
$$

Pelo corolário anterior concluímos que se a variável aleatória possui uma média pequena, então ela deve ser igual a 0 com alta probabilidade. Este método é uma poderosa ferramenta para provar resultados.

Teorema 3.3.3 (Desigualdade de Chebychev). Seja $X$ uma variável aleatória de valores inteiros com $\operatorname{Var}(X)=\sigma^{2}$. Temos que, para $a>0$,

$$
\mathbb{P}(|X-\mathbb{E}[X]| \geq a) \leq \frac{\sigma^{2}}{a^{2}} .
$$


Demonstração. Note que

$$
\mathbb{P}(|X-\mathbb{E}[X]| \geq a)=\mathbb{P}\left((X-\mathbb{E}[X])^{2} \geq a^{2}\right),
$$

e aplicando a desigualdade de Markov teremos

$$
\mathbb{P}(|X-\mathbb{E}[X]| \geq a) \leq \frac{\mathbb{E}\left[(X-\mathbb{E}[X])^{2}\right]}{a^{2}}=\frac{\sigma^{2}}{a^{2}} .
$$

Corolário 3.3.4 (Método do segundo momento). Seja $X$ uma variável aleatória de valores inteiros com $\operatorname{Var}(X)=\sigma^{2}$ e $\mathbb{E}[X] \geq m$, então

$$
\mathbb{P}(X=0) \leq \frac{\sigma^{2}}{m^{2}}
$$

Por (3.16) temos que se a variável aleatória possui uma média grande, e a variância é pequena comparada com o quadrado da média, então ela deve deve ser positiva com alta probabilidade. 


\section{MODELO DE GRAFO ALEATÓRIO NÃO HOMOGÊNEO}

Vamos apresentar com mais especificidade uma versão do modelo de grafo aleatório proposto por Kang, Pachón e Rodríguez (2016), que possuí como motivação a construção de um grafo aleatório com estrutura de comunidades. O modelo pode ser de grande interesse para modelar certas redes reais devido à sua versatilidade. Uma característica importante em grafos é a homogeneidade. Um grafo homogêneo possui uma padronização nos graus de seus vértices, em contrapartida um grafo não homogêneo estabelece diferentes probabilidades de graus para cada vértice em especial. O grafo binomial descrito no Capítulo 2 possui as mesmas probabilidade de obter um valor de grau para todos os vértices, logo, ele é homogêneo. Um fato interessante é que o modelo que será apresentado a seguir se utiliza do grafo binomial como base em sua construção mas possui a possibilidade de gerar um grafo aleatório não homogêneo, o que é bom no sentido de representar uma rede real, pois a maioria delas apresentam não homogeneidade (vértices com diferentes distribuições de probabilidade para suas conexões).

\subsection{Construção do modelo}

A criação do grafo é dada da seguinte forma: primeiramente geramos um grafo com modelo binomial $G(n, p)$, com $n$ sendo o numero de vértices e $p$ a probabilidade de conexão entre quaisquer dois vértices. Em seguida são estipulados subconjuntos de tamanhos arbitrários aos $n$ vértices. Cada subconjunto (aglomerado) é denominado de super-vértice de tamanho $i$, onde $i$ é a quantia de vértices pertencentes ao aglomerado. Estes aglomerados não possuem necessariamente vértices conectados. Por fim estipulamos novos vértices a partir dos super-vértices criados, sendo que cada super-vértice esta conectado com outro se havia pelo menos uma aresta entre os vértices dos subconjuntos em $G(n, p)$. Note que 
as conexões de $G(n, p)$ podem ser feitas após estipular os aglomerados, tendo o mesmo resultado, para melhor entendimento veja Figura 15 e Figura 16.
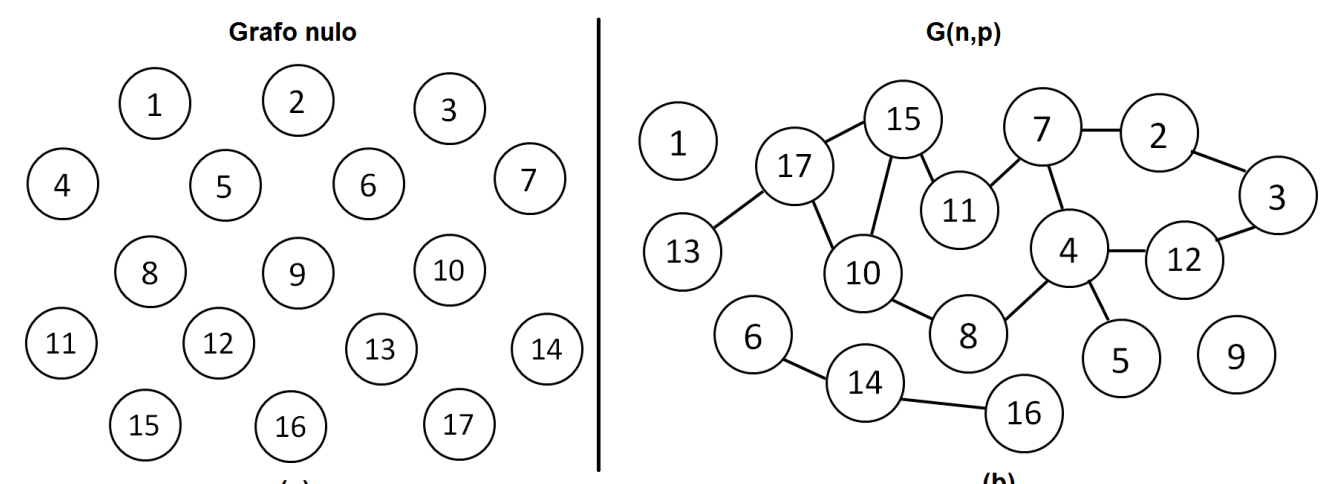

(b)

Figura 15 - (a) Iniciamos com um conjunto de $n$ vértices. (b) Criamos um grafo binomial $G(n, p)$.

Fonte: Elaborada pelo autor.
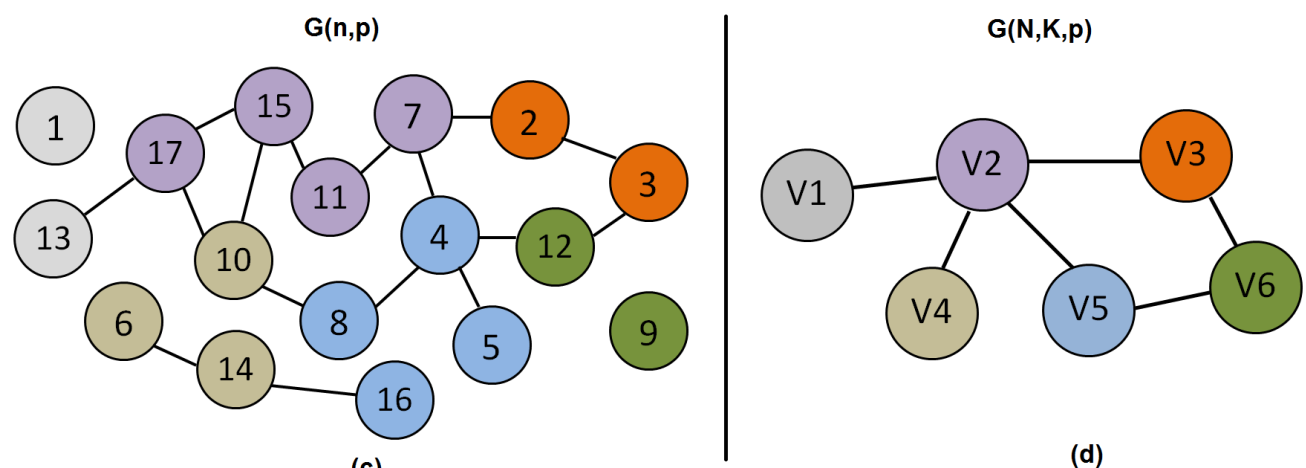

Figura 16 - (c) Estabelecemos uma configuração de comunidades de forma arbitrária. (d) Criamos supervértices com as comunidades e os conectamos de acordo com a conexão anterior em $G(n, p)$.

Fonte: Elaborada pelo autor.

Formalmente, sejam $N \in \mathbb{N}, r \in \mathbb{N}, \mathcal{K}=\left\{k_{i} ; k_{i} \in \mathbb{N}, 1 \leq i \leq r\right\}$ e $p \in(0,1)$ dados. É definido $G(N, \mathcal{K}, p)$ sendo um grafo aleatório tendo $N$ super-vértices com configuração $\mathcal{K}$, onde $k_{i}$ denota o número de super-vértices de tamanho $i$, para $i=1,2, \ldots, r$, no qual uma aresta está presente entre um par de super-vértices de tamanho $i$ e $j$ com probabilidade

$$
p_{i j}=1-(1-p)^{i j}
$$

independente umas das outras, e assumindo a não existência de loops (vértice conectado com ele mesmo). Note que a Expressão (4.1) é a probabilidade de existir ao menos uma aresta entre os vértices dos subconjuntos correspondentes em $G(n, p)$. Para obtermos esta probabilidade basta pensarmos no complementar da ligação entre dois super-vértices, ou seja, no evento onde nenhum dos vértices de um super-vértice de tamanho $i$ se conecta 
aos vértices de outro super-vértice de tamanho $j$. Lembrando que para cada dois vértices simples a probabilidade de não haver conexão é $1-p$. Em outras palavras,

$$
\begin{aligned}
p_{i j} & =1-\mathbb{P}(\text { de } i \text { vértices não estarem conectados com } j \text { vértices }) \\
& =1-[\underbrace{(1-p)^{j}(1-p)^{j}(1-p)^{j}(1-p)^{j}(1-p)^{j} \ldots(1-p)^{j}}_{i \text { vezes }}] \\
& =1-(1-p)^{i j} .
\end{aligned}
$$

Note que, desta forma, o número de super-vértices e o número de vértices são dados por

$$
N=\sum_{i=1}^{r} k_{i} \text { e } n=\sum_{i=1}^{r} i k_{i}
$$

respectivamente.

Como já mencionado, a homogeneidade de um grafo aleatório pode ser vista em relação aos graus dos vértices que o compõe. No caso do grafo binomial, todos os vértices possuem a mesma probabilidade de possuírem um determinado grau $k$, logo o modelo é dito homogêneo. Entretanto, no caso do modelo apresentado anteriormente, a distribuição dos graus podem se dar de forma bastante diferenciada dependendo da configuração que tomamos em relação ao tamanho dos super-vértices. Note que super-vértices de maior tamanho possuem probabilidade maior de se conectarem com os demais, ocasionando uma heterogeneidade entre os graus de super-vértices com tamanho diferentes. Devido a esta propriedade, podemos dizer que o modelo de grafo aleatório $G(N, \mathcal{K}, p)$ é não homogêneo.

\subsection{Resultados sobre conectividade}

O trabalho de Kang, Pachón e Rodríguez (2016) aborda diferentes propriedades para o modelo proposto. Um destas propriedades diz respeito ao limiar de conectividade do grafo para diferentes valores de $p$ em função de $n$ ou $N$. Nossos estudos serão focados nesta propriedade, mas com pequenas modificações, no âmbito de simplificar a forma de expor ao leitor.

Assume-se que, para $i=1,2, \ldots, r$ o seguinte limite exista

$$
\mu_{i}:=\lim _{N \rightarrow \infty} \frac{k_{i}}{N} .
$$

Isto implica que, quando $r \in \mathbb{N}$, o limite de $n / N$ existe e é dado por $\sum_{i=1}^{r} i \mu_{i}$. No propósito de simplificação, assumimos que $\mu_{1}>0$, o que significa que $k_{1}$ possui alguma proporção em relação a $N$.

Denotamos $c(N)$ por uma função de $N$ tal que

$$
-\ln (N)<c(N)<-\ln (N)+n,
$$


e definimos

$$
c_{*}:=\lim _{N \rightarrow \infty} c(N) \in[-\infty, \infty]
$$

Nosso enfoque dominante será o resultado sobre conectividade dado pelo seguinte teorema.

Teorema 4.2.1 (Limiar de Conectividade). Considere um grafo aleatório $G(N, \mathcal{K}, p)$ com $r \in \mathbb{N}$ e

$$
p:=\frac{\ln N+c(N)}{n}
$$

onde as condições (4.4) e $\mu_{1}>0$ são satisfeitas.

1. Se $c_{*}=-\infty$, então

$$
\lim _{N \rightarrow \infty} \mathbb{P}[G(N, \mathcal{K}, p) \text { ser conectado }]=0
$$

2. Se $c_{*}=c \in \mathbb{R}$ uma constante, então

$$
\lim _{N \rightarrow \infty} \mathbb{P}[G(N, \mathcal{K}, p) \text { ser conectado }]=e^{-\mu_{1} e^{-c}} .
$$

3. Se $c_{*}=+\infty$, então

$$
\lim _{N \rightarrow \infty} \mathbb{P}[G(N, \mathcal{K}, p) \text { ser conectado }]=1
$$

Note que tomando $N=n$ nós obtemos exatamente o modelo binomial, assim o Teorema 4.2.1 ficará igual ao Teorema 2.3.3. A demonstração do teorema se utiliza de argumentos descritos no Capítulo 2 e Capítulo 3, e em todas elas vamos precisar saber o comportamento do número de super-vértices isolados. O primeiro item do teorema é provado mostrando que, com alta probabilidade, existem super-vértices isolados, usando o método do segundo momento. O segundo item utiliza-se do método de Stein, estabelecendo um limite superior para a distância de variação total entre a variável aleatória que representa a quantidade de super-vértices isolados e uma distribuição de Poisson, verificando assim, que a variável segue assintoticamente uma lei de Poisson. Por fim, no terceiro item utilizamos da desigualdade de Markov e alguns conceitos de grafos da categoria árvores (Seção 2.2).

\subsection{Momentos do número de super-vértices isolados}

Iniciamos definindo a variável aleatória $X=X_{N}$ como sendo o número de supervértices isolados no grafo $G(N, \mathcal{K}, p)$, esta variável depende do valor $N$, mas iremos usar apenas $X$ para simplificar a notação. Com isso, vamos estabelecer expressões para $\mathbb{E}[X]$, $\mathbb{E}\left[X^{2}\right]$ e $\mathbb{E}^{2}[X]$ o que nos auxiliará nas demonstrações de lemas posteriores relacionados a conectividade do modelo. 
Veja que, para qualquer ordem arbitrária de super-vértices, podemos escrever:

$$
X=\sum_{i=1}^{r} \sum_{k=1}^{k_{i}} I_{k}^{i}
$$

onde $I_{k}^{i}=1$ se o k-ésimo super-vértice de tamanho $i$ é isolado, e 0 caso contrário.

Seja o evento $D_{j}^{i}=\{$ Um super-vértice fixo de tamanho $i$ não possui conexão com outro super-vértice fixo de tamanho $j$ \}. Para que um super-vértice seja isolado ele não pode possuir nenhuma conexão com qualquer outro super-vértice existente no grafo. Portanto, o evento $D_{j}^{i}$ precisa ocorrer para todos os $1 \leq j \leq r, k_{j}$ vezes. Como não consideramos a existência de auto-conexões, se tomarmos um super-vértice de tamanho $i$, o evento $D_{i}^{i}$ precisa ocorrer apenas $k_{i}-1$ vezes. Note que a probabilidade de $D_{j}^{i}$, é $1-p_{i j}$.

Como cada par de super-vértices é conectado independentemente, propriedade satisfeita devido a sua relação com as conexões dos vértices no modelo binomial $G(n, p)$, temos que,

$$
\begin{aligned}
\mathbb{E}\left(I_{k}^{i}\right) & =\mathbb{P}\left[I_{k}^{i}=1\right] \\
& =\mathbb{P}\left(\left\{D_{i}^{i}\right\}\right)^{k_{i}-1} \prod_{j \neq i, j=1}^{r} \mathbb{P}\left(\left\{D_{j}^{i}\right\}\right)^{k_{j}} \\
& =\left(1-p_{i i}\right)^{k_{i}-1} \prod_{j \neq i, j=1}^{r}\left(1-p_{i j}\right)^{k_{j}} \\
& =(1-p)^{i^{2}\left(k_{i}-1\right)} \prod_{j \neq i, j=1}^{r}(1-p)^{i j k_{j}} \\
& =(1-p)^{i^{2}\left(k_{i}-1\right)+i \sum_{j \neq i, j=1}^{r} j k_{j}} \\
& =(1-p)^{i(n-i)} .
\end{aligned}
$$

Obtendo assim, que

$$
\mathbb{E}(X)=\sum_{i=1}^{r} \sum_{k=1}^{k_{i}} \mathbb{E}\left(I_{k}^{i}\right)=\sum_{i=1}^{r} \sum_{k=1}^{k_{i}}(1-p)^{i(n-i)}=\sum_{i=1}^{r} k_{i}(1-p)^{i(n-i)} .
$$

Por outro lado, temos que

$$
\mathbb{E}\left(X^{2}\right)=\mathbb{E}\left(\left(\sum_{i=1}^{r} \sum_{k=1}^{k_{i}} I_{k}^{i}\right)^{2}\right)
$$

Podemos interpretar os produtos anteriores obtidos do elemento ao quadrado como arranjos tomados dois a dois, somando com o quadrado de cada vértice. Note que $\mathbb{E}\left[I_{1}^{i} I_{1}^{i}\right]=$ $\mathbb{E}\left[I_{1}^{i}\right]$, desta forma, temos:

- Para o produto dos mesmos vértices

$$
\sum_{i=1}^{r} \sum_{k=1}^{k_{i}} \mathbb{E}\left[\left(I_{k}^{i}\right)^{2}\right]=\sum_{i=1}^{r} \sum_{k=1}^{k_{i}} \mathbb{E}\left[I_{k}^{i}\right]=\mathbb{E}[X]
$$


- Para vértices diferentes de tamanhos iguais,

$$
\sum_{i=1}^{r} A_{k_{i}, 2} \mathbb{E}\left[I_{1}^{i} I_{2}^{i}\right]=\sum_{i=1}^{r}\left(k_{i}\right)\left(k_{i}-1\right) \mathbb{E}\left[I_{1}^{i} I_{2}^{i}\right]
$$

onde $A_{k_{i}, 2}$ é um arranjo de $k_{i}$ elementos tomados 2 a 2 , note que, se $k_{i}=1$ então não existem dois vértices diferentes do mesmo tamanho específico $i$, logo o elemento do somatório não irá existir.

- Para vértices diferentes de tamanhos diferentes

$$
\sum_{\substack{i, j=1 \\ i \neq j}}^{r} k_{i} k_{j} \mathbb{E}\left[I_{1}^{i} I_{2}^{j}\right]
$$

veja que o número subscrito 2 está apenas denotando que os vértices não são os mesmos, assim para $k_{j}=1$, tome $\mathbb{E}\left[I_{1}^{i} I_{1}^{j}\right]$.

Com isso, podemos escrever

$$
\mathbb{E}\left[X^{2}\right]=\mathbb{E}(X)+\sum_{i, j=1}^{r} k_{i} k_{j} \mathbb{E}\left[I_{1}^{i} I_{2}^{j}\right]-\sum_{i=1}^{r} k_{i} \mathbb{E}\left[I_{1}^{i} I_{2}^{i}\right]
$$

O calculo das esperanças para $i \neq j$ é dado por

$$
\begin{aligned}
\mathbb{E}\left[I_{1}^{i} I_{2}^{j}\right] & =\mathbb{P}\left[I_{1}^{i}=1, I_{2}^{j}=1\right] \\
& =\mathbb{P}\left[I_{1}^{i}=1 \mid I_{2}^{j}=1\right] \mathbb{P}\left[I_{2}^{j}=1\right] \\
& =\left(\left(1-p_{i i}\right)^{k_{i}-1}\left(1-p_{i j}\right)^{k_{j}-1} \prod_{l \neq j, i}\left(1-p_{i l}\right)^{k_{l}}\right) \mathbb{P}\left[I_{2}^{j}=1\right] \\
& =\left((1-p)^{i^{2}\left(k_{i}-1\right)}(1-p)^{i j\left(k_{j}-1\right)} \prod_{l \neq j, i}(1-p)^{i l\left(k_{l}\right)}\right)(1-p)^{j(n-j)} \text { devido a }(4.1), \\
& =(1-p)^{i^{2}\left(k_{i}-1\right)+i j\left(k_{j}-1\right)+\sum_{l \neq i, j} i k_{l}+j(n-j)} \text { devido a }(4.2) \\
& =(1-p)^{i(n-i)+j(n-j)-i j}
\end{aligned}
$$

Similarmente, se tomarmos $i=j$ teremos

$$
\mathbb{E}\left[I_{1}^{i} I_{2}^{i}\right]=(1-p)^{2 i(n-i)-i^{2}}
$$

Os próximos resultados utilizam de notação assintótica (Símbolos de Landau), veja o Apêndice B para mais detalhes. Sabemos que $1-x=e^{-x+O\left(x^{2}\right)}$ para $|x|<1$ (ver Exemplo 
B.0.1, Apêndice B). Como definimos $p \in(0,1)$ temos que:

$$
\begin{aligned}
(1-p)^{i n-i^{2}} & =e^{\left(-p+O\left(p^{2}\right)\right)\left(i n-i^{2}\right)} \\
& =e^{-i n p+i^{2} p+i n O\left(p^{2}\right)-i^{2} O\left(p^{2}\right)} \quad(\text { desconsiderando as constantes }) \\
& =e^{-i n p+O(p)+n O\left(p^{2}\right)+O\left(p^{2}\right)} \\
& =e^{-i(\ln (N)+c(N))+O\left(\max \left\{p, n p^{2}, p^{2}\right\}\right)} \\
& =e^{-i(\ln (N)+c(N))+O\left(n p^{2}\right)} \quad(\text { substituindo } p) \\
& =e^{-i(\ln (N)+c(N))+O\left(\frac{(\ln (N)+c(N))^{2}}{n}\right)}
\end{aligned}
$$

Dado que $e^{O\left(x^{2}\right)}=1+O\left(x^{2}\right)$ (ver Exemplo B.0.2, Apêndice B), obtemos

$$
(1-p)^{i n-i^{2}}=\left(1+O\left(\frac{(\ln (N)+c(N))^{2}}{n}\right)\right) e^{-i(\ln (N)+c(N))} .
$$

Temos então, por (4.8) e pela expressão anterior, que:

$$
\begin{aligned}
\mathbb{E}[X] & =\sum_{i=1}^{r} k_{i}(1-p)^{i n-i^{2}} \\
& =\sum_{i=1}^{r} k_{i} e^{-i(\ln (N)+c(N))}\left(1+O\left(\frac{(\ln (N)+c(N))^{2}}{n}\right)\right) \\
& =\left(1+O\left(\frac{(\ln (N)+c(N))^{2}}{n}\right)\right) \sum_{i=1}^{r} \frac{k_{i}}{e^{(\ln (N)+c(N)) i}} \\
& =\left(1+O\left(\frac{(\ln (N)+c(N))^{2}}{n}\right)\right) \sum_{i=1}^{r} \frac{k_{i}}{N^{i} e^{c(N) i}} .
\end{aligned}
$$

Para facilitar a notação, vamos definir:

$$
F_{N}=\left(1+O\left(\frac{(\ln (N)+c(N))^{2}}{n}\right)\right) .
$$

Logo, obtemos

$$
\mathbb{E}[X]=F_{N} \sum_{i=1}^{r} \frac{k_{i}}{N^{i} e^{c(N) i}}
$$

Agora vamos analisar $\mathbb{E}\left[X^{2}\right]$. Para isso, usando novamente que $1-x=e^{-x+O\left(x^{2}\right)}$ para $|x|<1$, vemos que:

$$
\begin{aligned}
(1-p)^{(i+j) n-(i+j)^{2}+i j} & =e^{\left[-(i+j) n p+(i+j)^{2} O(p)+(i+j) n O\left(p^{2}\right)-(i+j)^{2} O\left(p^{2}\right)+i j O\left(p^{2}\right)-i j O(p)\right]} \\
& =e^{-(i+j) n p+n O\left(p^{2}\right)} \\
& =e^{-(i+j)(\ln (N)+c(N))+O\left(n p^{2}\right)} \\
& =F_{N} e^{-(i+j)(\ln (N)+c(N))} \\
& =F_{N} \frac{1}{N^{(i+j)}} e^{(i+j) c(N)}
\end{aligned}
$$


E assim, pelas Equações (4.9)-(4.12) e (4.15) temos:

$$
\mathbb{E}\left[X^{2}\right]=\mathbb{E}[X]-F_{N}\left[\sum_{i=1}^{r} \frac{k_{i}}{N^{2 i} e^{c(N) 2 i}}-\sum_{i, j=1}^{r} \frac{k_{i} k_{j}}{N^{(i+j)} e^{(i+j) c(N)}}\right] .
$$

Para completar as informações que precisamos, temos para $\mathbb{E}^{2}[X]$ que:

$$
\begin{aligned}
\mathbb{E}^{2}[X] & =\left(F_{N}\right)^{2} \sum_{i=1}^{r} \frac{k_{i}}{N^{i} e^{c(N) i}} \sum_{j=1}^{r} \frac{k_{j}}{N^{j} e^{c(N) j}} \\
& =\left(F_{N}\right)^{2} \sum_{i, j=1}^{r} \frac{k_{i} k_{j}}{N^{(i+j)} e^{(i+j) c(N)}} .
\end{aligned}
$$

Note que, por propriedade de $O(x)$ (Apêndice B),

$$
(1+O(x))^{2}=1+2 O(x)+(O(x))^{2}=1+O(x)+O\left(x^{2}\right)=1+\max \left\{|O(x)|,\left|O\left(x^{2}\right)\right|\right\} .
$$

Como temos que, assumindo $c(N)<0$ ou $c(N)=c \in \mathbb{R}$ para $N$ suficientemente grande,

$$
\lim _{N \rightarrow \infty} O\left(\frac{(\ln (N)+c(N))^{2}}{n}\right) \geq \lim _{N \rightarrow \infty} O\left(\frac{(\ln (N)+c(N))^{2}}{n}\right)^{2},
$$

então podemos escrever que

$$
\mathbb{E}^{2}[X]=F_{N} \sum_{i, j=1}^{r} \frac{k_{i} k_{j}}{N^{(i+j)} e^{(i+j) c(N)}}
$$

\subsection{Existência de super-vértices isolados}

Vamos analisar o comportamento assintótico da probabilidade de $X=0$ em relação à $c_{*}$ utilizando do lema apresentado a seguir.

Lema 2. Considere o grafo aleatório $G(N, \mathcal{K}, p) \operatorname{com} r \in \mathbb{N}, \mu_{1}>0$ e $p=\frac{\ln (N)+c(N)}{n}$.

1. Se $c_{*}=-\infty$, então

$$
\lim _{N \rightarrow \infty} \mathbb{P}(X=0)=0
$$

2. Se $c_{*}=\infty$, então

$$
\lim _{N \rightarrow \infty} \mathbb{P}(X=0)=1
$$

Demonstração. Item 1.

Para provarmos este item, iremos usar o método do segundo momento (Corolário 3.3.4, Seção 3.3). De fato, como $\mathbb{E}[X] \geq \mathbb{E}[X]$, temos que

$$
\lim _{N \rightarrow \infty} \mathbb{P}(X=0) \leq \lim _{N \rightarrow \infty}\left(\frac{\mathbb{V}[X]}{\mathbb{E}^{2}[X]}\right)=\lim _{N \rightarrow \infty}\left(\frac{\mathbb{E}\left[X^{2}\right]}{\mathbb{E}^{2}[X]}-\frac{\mathbb{E}^{2}[X]}{\mathbb{E}^{2}[X]}\right) .
$$


Sendo assim, se provarmos que o ultimo limite converge para 0 , teremos demonstrado o item 1.

Usando dos cálculos para $\mathbb{E}[X]$, note que os elementos do somatório em (4.13) são todos não negativos, e assim podemos escrever

$$
\begin{aligned}
\sum_{i=1}^{r} \frac{k_{i}}{N^{i} e^{c(N) i}} & =\frac{k_{1}}{N e^{c(N)}}+\sum_{i>1}^{r} \frac{k_{i}}{N^{i} e^{c(N) i}} \\
& \geq \frac{k_{1}}{N e^{c(N)}}
\end{aligned}
$$

Ao tomar $\lim _{N \rightarrow \infty} F_{N}$ obtemos um valor entre 1 e $\infty$. Assim, usando a desigualdade anterior, e uma vez que $c_{*}=-\infty$, teremos:

$$
\mathbb{E}[X] \geq F_{N} \frac{k_{1}}{N e^{c(N)}} \rightarrow \infty \text { quando } N \rightarrow \infty,
$$

ou seja, $\lim _{N \rightarrow \infty} \mathbb{E}[X]=\infty$.

Partimos agora, usando das informações anteriores, para a análise do comportamento de $\mathbb{E}\left[X^{2}\right] / \mathbb{E}^{2}[X]$.

$$
\begin{aligned}
\frac{\mathbb{E}\left[X^{2}\right]}{\mathbb{E}^{2}[X]} & =\frac{\mathbb{E}[X]-F_{N}\left[\sum_{i=1}^{r} \frac{k_{i}}{N^{2 i} e^{c(N) 2 i}}-\sum_{i, j=1}^{r} \frac{k_{i} k_{j}}{N^{(i+j)} e^{(i+j) c(N)}}\right]}{\mathbb{E}^{2}[X]} \\
& =\frac{1}{\mathbb{E}[X]}-\frac{F_{N}\left[\sum_{i=1}^{r} \frac{k_{i}}{N^{2 i} e^{c(N) 2 i}}-\sum_{i, j=1}^{r} \frac{k_{i} k_{j}}{N^{(i+j)} e^{(i+j) c(N)}}\right]}{F_{N} \sum_{i, j=1}^{r} \frac{k_{i} k_{j}}{N^{(i+j)} e^{(i+j) c(N)}}} .
\end{aligned}
$$

Note que

$$
\frac{\sum_{i=1}^{r} \frac{k_{i}}{N^{2 i} e^{c(N) 2 i}}}{\sum_{i, j=1}^{r} \frac{k_{i} k_{j}}{N^{(i+j)} e^{(i+j) c(N)}}}=\frac{\sum_{i=1}^{r} \frac{k_{i}}{N^{i} e^{c(N) i}} \frac{e^{-c(N) i}}{N^{i}}}{\sum_{i=1}^{r} \frac{k_{i}}{N^{i} e^{c(N) i}} \sum_{j=1}^{r} \frac{k_{j}}{N^{j} e^{c(N) j}}} .
$$

Como, por definição $c(N)>-\ln N$, $\log 0 e^{-c(N)}<e^{\ln N}$ e assim podemos escrever

$$
\begin{aligned}
\frac{\sum_{i=1}^{r} \frac{k_{i}}{N^{2 i} e^{((N) 2 i}}}{\sum_{i, j=1}^{r} \frac{k_{i} k_{j}}{N^{(i+j)} e^{(i+j) c(N)}}} & <\frac{\sum_{i=1}^{r} \frac{k_{i}}{N^{i} e^{c(N) i}} \frac{e^{\ln N^{i}}}{N^{i}}}{\sum_{i=1}^{r} \frac{k_{i}}{N^{i} e^{(N) i}} \sum_{j=1}^{r} \frac{k_{j}}{N^{j} e^{c(N) j}}} \\
& =\frac{1}{\sum_{j=1}^{r} \frac{k_{j}}{N^{j} e^{c(N) j}}} .
\end{aligned}
$$

Mas, pelo mesmo fato usado na expressão (4.18) onde calculamos a média de $X$, temos que

$$
\sum_{j=1}^{r} \frac{k_{j}}{N^{j} e^{c(N) j}}>\frac{k_{1}}{N} e^{-c(N)} \rightarrow \infty \text { quando } N \rightarrow \infty
$$

Logo

$$
\frac{\sum_{i=1}^{r} \frac{k_{i}}{N^{2 i} e^{c(N) 2 i}}}{\sum_{i, j=1}^{r} \frac{k_{i} k_{j}}{N^{(i+j)} e^{(i+j) c(N)}}}<\frac{1}{\sum_{j=1}^{r} \frac{k_{j}}{N^{j} e^{c(N) j}}} \rightarrow 0 \text { quando } N \rightarrow \infty .
$$


Veja que, para $c(N) \rightarrow-\infty, c(N)<0$ para algum $N$ suficientemente grande, então

$$
\begin{aligned}
\lim _{N \rightarrow \infty} F_{N} & =\lim _{N \rightarrow \infty}\left(1+O\left(\frac{(\ln (N)+c(N))^{2}}{n}\right)\right) \\
& <\lim _{N \rightarrow \infty}\left(1+O\left(\frac{(\ln (N))^{2}}{n}\right)\right) \\
& <\lim _{N \rightarrow \infty}\left(1+O\left(\frac{(\ln (N))^{2}}{N}\right)\right)=1 .
\end{aligned}
$$

A ultima expressão é devido a propriedade de que se $x \rightarrow 0$ então $O(x) \rightarrow 0$, e como $\lim _{N \rightarrow \infty} \frac{(\ln (N))^{2}}{N} \rightarrow 0$ por L'Hospital, a Equação (4.19) é satisfeita.

Concluímos assim, que

$$
\begin{aligned}
\lim _{N \rightarrow \infty} \frac{\mathbb{E}\left[X^{2}\right]}{\mathbb{E}^{2}[X]} & =\lim _{N \rightarrow \infty}\left(\frac{1}{\mathbb{E}[X]}-\frac{F_{N} \sum_{i=1}^{r} \frac{k_{i}}{N^{2 i} e^{c(N) 2 i}}}{F_{N} \sum_{i, j=1}^{r} \frac{k_{i} k_{j}}{N^{(i+j)} e^{(i+j) c(N)}}}+\frac{\left.F_{N} \sum_{i, j=1}^{r} \frac{k_{i} k_{j}}{N_{N} \sum_{i, j=1}^{r} \frac{k_{i} k_{j}}{N^{(i+j)} e^{(i+j) c(N)}}}\right)}{F^{(i+j) c(N)}}\right) \\
& =0-0+1 \\
& =1 .
\end{aligned}
$$

Desta forma, como já mencionado, podemos usar o método do segundo momento, verificando que

$$
\lim _{N \rightarrow \infty} \mathbb{P}(X=0) \leq \lim _{N \rightarrow \infty}\left(\frac{\mathbb{V}[X]}{\mathbb{E}^{2}[X]}\right)=\lim _{N \rightarrow \infty}\left(\frac{\mathbb{E}\left[X^{2}\right]}{\mathbb{E}^{2}[X]}-\frac{\mathbb{E}^{2}[X]}{\mathbb{E}^{2}[X]}\right)=1-1=0,
$$

como queríamos demonstrar. Tendo assim provado o item 1 do Lema 2.

\section{Item 2.}

Nesta prova iremos usar a desigualdade de Markov apresentada na Seção 3.3. Com efeito, se $\mathbb{E}[X]<\infty$, então

$$
\lim _{N \rightarrow \infty} \mathbb{P}(X \geq 1) \leq \lim _{N \rightarrow \infty} \frac{\mathbb{E}[X]}{1} .
$$

Logo, se provarmos que $\mathbb{E}(X) \rightarrow 0$ quando $N \rightarrow \infty$, podemos usar o complementar desta informação para provar o item 2.

Temos por (4.14) que

$$
\begin{aligned}
\mathbb{E}[X] & =F_{N} \sum_{i=1}^{r} \frac{k_{i}}{N^{i} e^{c(N) i}} \\
& \leq F_{N} \frac{1}{e^{c(N)}} \sum_{i=1}^{r} \frac{k_{i}}{N^{i}}
\end{aligned}
$$

Como $k_{i}<N$ para qualquer $i$, então $k_{i}<N^{i}$, logo temos que $\sum_{i=1}^{r} \frac{k_{i}}{N^{i}}<\infty$.

Vamos analisar agora $F_{N} e^{-c(N)}$. Como $O(x) \leq J x$ para alguma constante positiva $J \in \mathbb{R}$, temos que

$$
\left(1+O\left(\frac{(\ln (N)+c(N))^{2}}{n}\right)\right) \frac{1}{e^{c(N)}} \leq\left(1+J\left(\frac{(\ln (N)+c(N))^{2}}{n}\right)\right) \frac{1}{e^{c(N)}},
$$


que é igual a

$$
e^{-c(N)}+J e^{-c(N)} \frac{(\ln (N)+c(N))^{2}}{n} .
$$

Note que $e^{-c(N)}$ converge para 0 , pois neste item estamos considerando que $c(N) \rightarrow$ $\infty$ quando $N \rightarrow \infty$.

Para o segundo elemento da soma em (4.20) vamos fazer a seguinte análise. Considere a função

$$
f(x)=\frac{J(\ln (N)+x)^{2}}{N e^{x}} .
$$

Note que sua derivada é dada por

$$
\frac{d}{d x}(f(x))=-\frac{J e^{-x}(\ln (N)+x-2)(\ln (N)+x)}{N} .
$$

Com isso, temos que para qualquer $N>e^{2}$ e $J, x>0$, a derivada anterior resulta em um valor negativo, ou seja, nestas ocasiões a função é estritamente decrescente (um exemplo é apresentado na Figura 17). Sendo assim, tomando $x=c(N)$, para qualquer $N \geq 8, J \in \mathbb{R}$ positivo temos que o ponto máximo de $f(c(N))$ é quando $c(N)=0$. Obtemos com isso que,

$$
\lim _{N \rightarrow \infty} J e^{-c(N)} \frac{(\ln (N)+c(N))^{2}}{n} \leq \lim _{N \rightarrow \infty} J \frac{(\ln (N)+c(N))^{2}}{N e^{c(N)}} \leq \lim _{N \rightarrow \infty} J \frac{(\ln (N))^{2}}{N}=0,
$$

para qualquer $J$ positivo. Note que a desigualdade é sempre satisfeita pois podemos considerar $c(N)>0$, devido ao fato de que $c(N) \rightarrow+\infty$ quando $N \rightarrow \infty$.

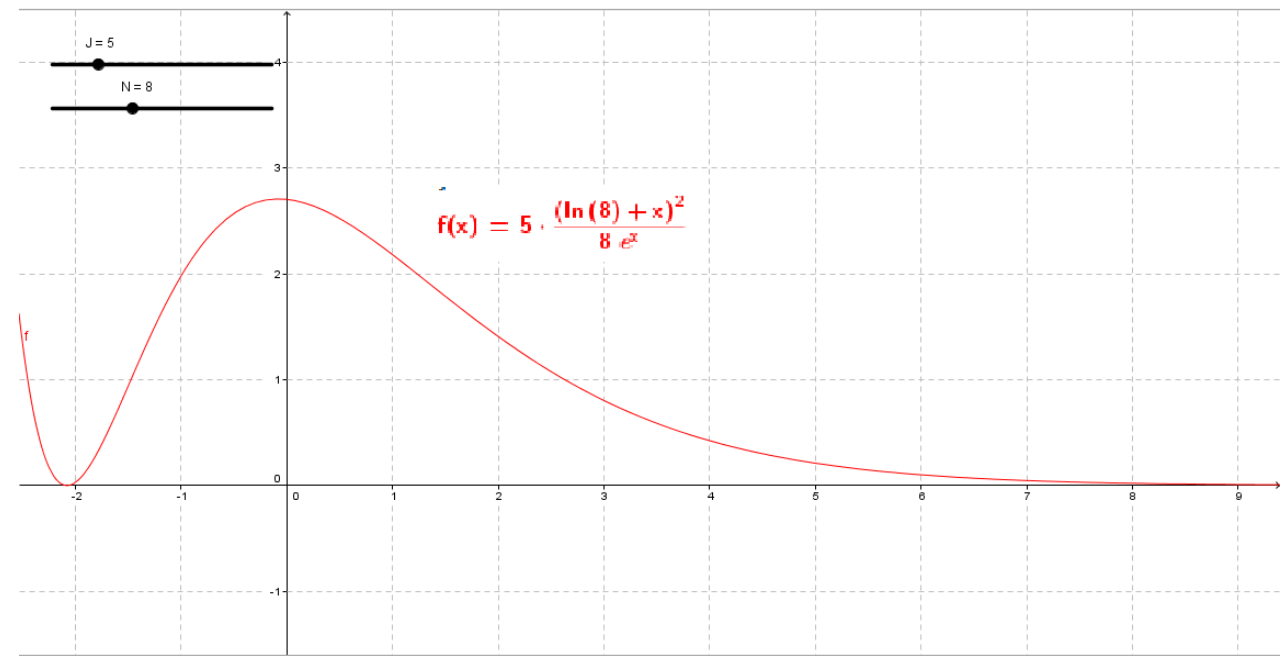

Figura 17 - Função $f(x)$ com $J=5$ e $N=8$

Fonte: Elaborada pelo autor.

Dadas as convergências, descobrimos que

$$
\lim _{N \rightarrow \infty} \mathbb{E}[X] \leq \lim _{N \rightarrow \infty}\left(F_{N} \frac{1}{e^{c(N)}} \sum_{i=1}^{r} \frac{k_{i}}{N^{i}}\right)=0 .
$$


Para finalizar, como já mencionado, usamos a desigualdade de Markov. Como $\mathbb{E}[X]<\infty$ então

$$
\lim _{N \rightarrow \infty} \mathbb{P}(X \geq 1) \leq \lim _{N \rightarrow \infty} \frac{\mathbb{E}[X]}{1}=0
$$

E portanto, pelo complementar da probabilidade temos que

$$
\lim _{N \rightarrow \infty} \mathbb{P}(X=0)=1
$$

Completando a prova para o Lema 2.

\subsection{Distribuição do número de super-vértices isolados}

Anteriormente verificamos o comportamento para $\lim _{n \rightarrow \infty} \mathbb{P}(X=0)$ quando $c_{*}=$ $-\infty$ ou $c_{*}=\infty$. Agora iremos analisar o comportamento do mesmo limite mas para $c_{*}=$ $c \in \mathbb{R}$.

Lema 3. Considere o grafo aleatório $G(N, \mathcal{K}, p)$ com $r \in \mathbb{N}, \mu_{1}>0$ e $p=\frac{\ln (N)+c(N)}{n}$. Se $c_{*}=c \in \mathbb{R}$, então

$$
\lim _{N \rightarrow \infty} \mathbb{P}(X=0)=e^{-\mu_{1} e^{-c}}
$$

Nesta ocasião, poderíamos demonstrar este lema utilizando-se dos teoremas envolvendo momentos fatoriais, apresentados na Seção 3.1. Para isso, iríamos estabelecer a variável $X$ como sendo uma soma de indicadoras (o que foi feito posteriormente), e assim encontrar uma expressão para a esperança de $X$ usando uma soma de esperanças de funções indicadoras. Caso estas indicadoras fossem independentes, $X$ seria considerado uma binomial, sendo possível encontrarmos facilmente os momentos fatoriais. Em seguida poderíamos comparar os momentos fatoriais obtidos com os momentos fatoriais de uma distribuição de Poisson com o parâmetro $\mu_{1} e^{-c}$. O problema é que estas indicadoras não são independentes, o que acaba dificultando drasticamente a obtenção de uma expressão para os momentos fatoriais de $X$, mesmo utilizando-se de um somatório de índices distintos apresentado no Teorema 3.1.5. Devido a isso, vamos optar por outra forma de demonstração, que é dada a seguir.

Para demonstrar o Lema 3 vamos aplicar o Método de Stein (Lema 1) já apresentado no Capítulo 3.

Demonstração. Sem perda de generalidade vamos tomar $c(N)=c \in \mathbb{R}$.

Como vimos anteriormente em (4.8) temos que

$$
\mathbb{E}[X]=\sum_{i=1}^{r} k_{i}(1-p)^{i(n-i)}
$$


Vamos definir

$$
\begin{aligned}
\lambda^{*}:=\lim _{N \rightarrow \infty} \mathbb{E}[X] & =\lim _{N \rightarrow \infty} \sum_{i=1}^{r} k_{i}(1-p)^{i(n-i)} \\
& =\lim _{N \rightarrow \infty}\left(1+O\left(\frac{(\ln (N)+c)^{2}}{n}\right)\right) \sum_{i=1}^{r} \frac{k_{i}}{N^{i} e^{c i}} \text { por } \\
& =\lim _{N \rightarrow \infty} \sum_{i=1}^{r} \frac{k_{i}}{N^{i} e^{c i}} \\
& =\lim _{N \rightarrow \infty} \frac{k_{1}}{N} e^{-c}+\lim _{N \rightarrow \infty} \sum_{i=2}^{r} \frac{k_{i}}{N^{i} e^{c i}}
\end{aligned}
$$

Usando o fato de que $k_{i} \leq N$ para todo $i$, temos que

$$
\lim _{N \rightarrow \infty} \sum_{i=2}^{r} \frac{k_{i}}{N^{i} e^{c i}} \leq \lim _{N \rightarrow \infty} \sum_{i=2}^{r} \frac{1}{N^{i-1}}=0 .
$$

Logo,

$$
\lambda^{*}=\lim _{N \rightarrow \infty} \mathbb{E}[X]=\mu_{1} e^{-c}
$$

Note que podemos definir a v.a. $X$ como sendo uma soma de variáveis indicadoras de cada super-vértice do grafo, ou seja, dos $N$ super-vértices existentes, tendo assim

$$
X:=\sum_{z=1}^{N} I_{z}
$$

onde $I_{z}=1$ se o z-ésimo super-vértice é isolado e $I_{z}=0$ caso contrário, com uma ordem aleatória.

Assim, podemos escrever

$$
\mathbb{E}[X]=\sum_{z=1}^{N} \mathbb{E}\left[I_{z}\right]=N \mathbb{E}\left[I_{z}\right],
$$

o que nor resulta em

$$
\mathbb{E}\left[I_{z}\right]=\sum_{i=1}^{r} \frac{k_{i}(1-p)^{i(n-i)}}{N} .
$$

Vamos denotar $G_{z}(N, \mathcal{K}, p)$ como sendo o grafo $G(N, \mathcal{K}, p)$ mas com todas as arestas do z-ésimo vértice removidas. Seja $J_{l}^{z}=1$ se o l-ésimo super-vértice é isolado em $G_{z}(N, \mathcal{K}, p)$, e $J_{l}^{z}=0$ caso contrário. Podemos interpretar $J_{l}^{z}$ usando das funções indicadoras anteriores, pois ele informa se o l-ésimo super-vértice é isolado ou não, tendo condicionado que o z-ésimo super-vértice é isolado, ou seja,

$$
J_{l}^{z}=\left(I_{l} \mid I_{z}=1\right) .
$$

Utilizando da função $\mathcal{L}($.$) comentada em Seção 3.2, onde \mathcal{L}\left(\left\{Y_{l}\right\}_{l}\right)$ denota a distribuição conjunta das variáveis aleatórias $\left\{Y_{l}\right\}_{l}$, podemos escrever

$$
\mathcal{L}\left(\left\{J_{l}^{z}\right\}_{l}\right)=\mathcal{L}\left(\left\{I_{l}\right\}_{l} \mid I_{z}=1\right) .
$$


Vejamos agora que, se o l-ésimo super-vértice for isolado em $G(N, \mathcal{K}, p)$, ou seja, $I_{l}=1$, então não importa se retirarmos as arestas de qualquer outro super-vértice $z$, o l-ésimo super-vértice ainda será isolado, fazendo com que $J_{l}^{z}=1$ para todo $l \neq z$. Por outro lado, se $I_{l}=0$, então $J_{l}^{z}=0$ caso o l-ésimo super-vértice não seja conectado com o z-ésimo super vértice, ou $J_{l}^{z}=1$ caso o l-ésimo super-vértice tenha apenas conexão com o super-vértice $z$. Fazendo com que $J_{l}^{z}$ tome valores 0 ou 1. Em ambos os casos obtemos que $J_{l}^{z} \geq I_{l}$ para todo $l \neq z$. Logo, pela Definição 3.2 .3 as variáveis aleatórias $\left\{I_{z}\right\}_{z}$ são positivamente relacionadas, sendo assim possível aplicarmos o Lema 1

O Lema 1 nos fornece um limite superior para a distância de variação total entre uma v.a. $X$ e uma v.a. com distribuição $\operatorname{Poi}(\lambda)$, onde $\lambda=\mathbb{E}[X]$. Lembrando que $X=X_{N}$ $(X$ depende de $N)$, vamos usar este limite para mostrar que $d_{T V}\left(X, \operatorname{Poi}\left(\lambda_{N}\right)\right) \rightarrow 0$ quando $N \rightarrow \infty$. Para isso, iremos comprovar que $\mathbb{V}(X) / \mathbb{E}[X] \rightarrow 1$ e $\max _{1 \leq z \leq N}\left\{\mathbb{E}\left[I_{z}\right]\right\} \rightarrow 0$. Usando das Equações (4.16) e (4.17) temos que

$$
\begin{aligned}
\frac{\mathbb{V}(X)}{\mathbb{E}[X]} & =\frac{\mathbb{E}[X]-F_{N}\left[\sum_{i=1}^{r} \frac{k_{i}}{N^{2 i} e^{c 2 i}}-\sum_{i, j=1}^{r} \frac{k_{i} k_{j}}{N^{(i+j)} e^{(i+j) c}}\right]-F_{N} \sum_{i, j=1}^{r} \frac{k_{i} k_{j}}{N^{(i+j)} e^{(i+j) c}}}{\mathbb{E}[X]} \\
& =1-\frac{F_{N} \sum_{i=1}^{r} \frac{k_{i}}{N^{2 i} e^{c 2 i}}}{F_{N} \sum_{i=1}^{r} \frac{k_{i}}{N^{i} e^{c i}}} \\
& \leq 1-\frac{\frac{1}{N^{r} e^{c r}} F_{N} \sum_{i=1}^{r} \frac{k_{i}}{N^{i} e^{c i}}}{F_{N} \sum_{i=1}^{r} \frac{k_{i}}{N^{i} e^{c i}}} \\
& =1-\frac{1}{N e^{c}} \rightarrow 1 \text { quando } N \rightarrow \infty .
\end{aligned}
$$

Consideramos a sequência de variáveis aleatórias indicadoras $\left\{I_{z}\right\}_{z}$. Temos que para cada $z=1,2, \ldots, N$,

$$
\mathbb{E}\left[I_{z}\right]=\sum_{i=1}^{r} \frac{k_{i}(1-p)^{i(n-i)}}{N}=\frac{\mathbb{E}[X]}{N} \rightarrow 0 \text { quando } N \rightarrow \infty .
$$

Assim, obtemos

$$
\max _{1 \leq z \leq N}\left\{\mathbb{E}\left[I_{z}\right]\right\} \rightarrow 0 \text { quando } N \rightarrow \infty
$$

Concluímos então, que $d_{T V}\left(X, \operatorname{Poi}\left(\lambda_{N}\right)\right) \rightarrow 0$ quando $N \rightarrow \infty$.

Note que,

$$
d_{T V}\left(X, \operatorname{Poi}\left(\lambda^{*}\right)\right) \leq d_{T V}\left(X, \operatorname{Poi}\left(\lambda_{N}\right)\right)+d_{T V}\left(\operatorname{Poi}\left(\lambda_{N}\right), \operatorname{Poi}\left(\lambda^{*}\right)\right) .
$$

E como $\lim _{N \rightarrow \infty} d_{T V}\left(X, \operatorname{Poi}\left(\lambda_{N}\right)\right)=0$ e $\lim _{N \rightarrow \infty} d_{T V}\left(\operatorname{Poi}\left(\lambda_{N}\right), \operatorname{Poi}\left(\lambda^{*}\right)\right)=0$ temos que

$$
d_{T V}\left(X, \operatorname{Poi}\left(\lambda^{*}\right)\right) \rightarrow 0 \text { quando } N \rightarrow \infty .
$$

Logo, podemos concluir que $X$ converge assintoticamente para uma variável aleatória de Poisson com média $\lambda^{*}=\mu_{1} e^{-c}$ quando $N \rightarrow \infty$. 


\subsection{Componentes pequenos}

Nesta seção vamos analisar o comportamento assintótico da probabilidade de existência de componentes no grafo $G(N, \mathcal{K}, p)$ de tamanho $m$ para $2 \leq m \leq N / 2$, utilizando do seguinte lema:

Lema 4. Considere o grafo $G(N, \mathcal{K}, p)$ com $r \in \mathbb{N}, p$ definido por (4.5) e $c_{*}=\infty$ ou $c_{*}=c$, onde $c \in \mathbb{R}$. Então, com alta probabilidade, $G(N, \mathcal{K}, p)$ não possui componentes de tamanho $m$, com $2 \leq m \leq N / 2$.

Demonstração. Seja $\mathcal{S}^{m}$ o conjunto de todos os subconjuntos de $m$ super-vértices, e para cada $\mathcal{S} \in \mathcal{S}^{m}$, seja $m_{i}(\mathcal{S})$ o número de super-vértices de tamanho $i$ em $\mathcal{S}$. Note que

$$
m=m_{1}(\mathcal{S})+m_{2}(\mathcal{S})+\ldots+m_{r}(\mathcal{S}) .
$$

Observe que se os super-vértices em $\mathcal{S}$ formam um componente em $G(N, \mathcal{K}, p)$, então os dois seguintes eventos devem ocorrer:

1. $A_{1}^{\mathcal{S}}:=\{$ os super-vértices em $\mathcal{S}$ são conectados $\}$;

2. $A_{2}^{\mathcal{S}}:=\left\{\right.$ não há super-vértices em $\mathcal{S}$ conectados com super-vértices em $\left.\mathcal{S}^{c}\right\}$,

onde $\mathcal{S}^{c}$ denota o complementar de $\mathcal{S}$.

Note que a ocorrência de $A_{1}^{\mathcal{S}}$ depende apenas da conexão entre os super-vértices de dentro do conjunto $\mathcal{S}$, e a ocorrência de $A_{2}^{\mathcal{S}}$ depende apenas da conexão do supervértices de $\mathcal{S}$ com $\mathcal{S}^{c}$. Como a ocorrência destes eventos depende, respectivamente, de conjuntos disjuntos de elos, temos que $A_{1}^{\mathcal{S}}$ e $A_{2}^{\mathcal{S}}$ são independentes. Entretanto eles não são mutuamente exclusivos.

Sendo assim, podemos escrever

$$
\{\exists \text { um componente de tamanho } m\}=\bigcup_{\mathcal{S}^{m}}\left\{A_{1}^{\mathcal{S}} \cap A_{2}^{\mathcal{S}}\right\}
$$

e tomando a probabilidade temos que

$$
\begin{aligned}
\mathbb{P}(\exists \text { um componente de tamanho } m) & =\mathbb{P}\left(\bigcup_{\mathcal{S}^{m}}\left\{A_{1}^{\mathcal{S} \cap} \cap A_{2}^{\mathcal{S}}\right\}\right) \\
& \leq \sum_{\mathcal{S} \in \mathcal{S}^{m}} \mathbb{P}\left(A_{1}^{\mathcal{S}}\right) \mathbb{P}\left(A_{2}^{\mathcal{S}}\right)
\end{aligned}
$$

Para prosseguirmos, vamos utilizar de conceitos relacionados a classe de grafos denominados de árvores (ver Seção 2.2).

Veja que, um componente de tamanho $m$ contêm ao menos uma árvore de ordem $m$. Temos pelo Teorema de Cayley 2.2.2 que para grafos com $m$ vértices, é possível formar 
$m^{m-2}$ árvores distintas. Além disso, verificamos que

$$
\begin{aligned}
(1-p)^{i j} & =\sum_{k=0}^{i j}\left(\begin{array}{c}
i j \\
k
\end{array}\right)(-p)^{k} \\
& =1-i j p+\left(\begin{array}{c}
i j \\
2
\end{array}\right) p^{2}-\left(\begin{array}{c}
i j \\
3
\end{array}\right) p^{3}+\ldots \\
& \geq 1-i j p .
\end{aligned}
$$

E assim,

$$
\begin{aligned}
-1+(1-p)^{i j} & \geq-i j p \\
1-(1-p)^{i j} & \leq i j p \\
p_{i j} & \leq i j p \leq r^{2} p
\end{aligned}
$$

Usando as informações anteriores, e também o fato de que o evento

$$
A_{1}^{\mathcal{S}}=\{\mathcal{S} \text { contêm uma árvore de tamanho } m\}
$$

temos que

$$
\mathbb{P}\left(A_{1}^{\mathcal{S}}\right)=\mathbb{P}(\mathcal{S} \text { contêm uma árvore de tamanho } m) \leq m^{m-2}\left(r^{2} p\right)^{m-1} .
$$

Vamos definir, para cada $\mathcal{S} \in \mathcal{S}^{m}$,

$$
M_{m}(\mathcal{S})=\sum_{i=1}^{r} i m_{i}(\mathcal{S})
$$

o número de vértices que estão compondo os super-vértices em $\mathcal{S}$, ou seja, a soma dos tamanhos dos super-vértices de $\mathcal{S}$. Observe que o conjunto de $m$ super-vértices onde $A_{2}^{\mathcal{S}}$ ocorre, pode ser considerado como um único super-vértice isolado de tamanho $M_{m}(\mathcal{S})$. Logo, pela equação (4.7), temos que

$$
\mathbb{P}\left(A_{2}^{\mathcal{S}}\right)=(1-p)^{M_{m}(\mathcal{S})\left(n-M_{m}(\mathcal{S})\right)}
$$

Analisando a função $f(x)=x(n-x)=-x^{2}+x n$ para $x \in(0, n)$, temos que ela é uma função quadrática com concavidade para baixo, possui máximo global no ponto $x=n / 2$ e é simétrica em torno do máximo (veja Figura 18).

Note que para cada $\mathcal{S} \in \mathcal{S}^{m}$, se $x=M_{m}(\mathcal{S})$ então $x \geq m$. Assim,

$$
\begin{aligned}
m & \leq x \\
m n-m^{2} & \leq x n-m^{2} \\
m(n-m) & \leq x n-m^{2}+x^{2}-x^{2} \\
m(n-m) & \leq x n-x^{2}+\left(x^{2}-m^{2}\right)
\end{aligned}
$$




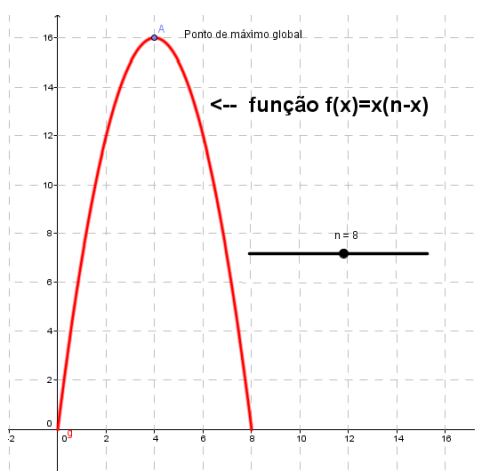

Figura 18 - Função $\mathrm{f}(\mathrm{x})=\mathrm{x}(\mathrm{n}-\mathrm{x})$, para $n=8, \quad x \in(0, n)$.

Fonte: Elaborada pelo autor.

minimizando um dos $x$ do lado direito da inequação,

$$
\begin{aligned}
& m(n-m) \leq x n-x^{2}+\left(m^{2}-m^{2}\right) \\
& m(n-m) \leq x(n-x) .
\end{aligned}
$$

Possibilitando assim, a escrevermos a inequação

$$
\mathbb{P}\left(A_{2}^{\mathcal{S}}\right) \leq(1-p)^{m(n-m)}
$$

Logo, por (4.22)-(4.24), obtemos

$\mathbb{P}(\exists$ um componente de tamanho $m) \leq\left(\begin{array}{l}N \\ m\end{array}\right) m^{m-2}\left(r^{2} p\right)^{(m-1)}(1-p)^{m(n-m)}$.

Lembramos que $(1-p)<e^{-p}$ para $p \in(0,1)$, e temos pela fórmula de Stirling (veja Apêndice B) que

$$
m ! \approx \sqrt{2 \pi} m^{m+1 / 2} e^{-m}
$$

Assim,

$$
\begin{aligned}
\left(\begin{array}{l}
N \\
m
\end{array}\right) & =\frac{N !}{(N-m) ! m !} \\
& \approx \frac{N ! e^{m}}{(N-m) ! \sqrt{2 \pi} m^{m+1 / 2}} \\
& \approx \frac{\sqrt{2 \pi} N ! e^{m}}{2 \pi(N-m) ! m^{m+1 / 2}}
\end{aligned}
$$

Como $\frac{\sqrt{2 \pi}}{2 \pi}<1$ e $\frac{N !}{(N-m) !} \leq(2 N)^{m}$ temos que

$$
\left(\begin{array}{l}
N \\
m
\end{array}\right)<\frac{(2 N e)^{m}}{m^{m+1 / 2}}
$$


Concluímos que a probabilidade de existir um componente de tamanho $m$ é menor que

$$
\frac{(2 N e)^{m}}{m^{m+1 / 2}} m^{m-2}\left(r^{2} p\right)^{m-1} e^{-p m(n-m)}
$$

para valores de $N$ suficientemente grandes. Podemos reescrever a expressão anterior como

$$
(2 N e)^{m} m^{m-2-m-1 / 2}\left(r^{2} p\right)^{m-1} e^{-p m(n-m)}=(2 N e)^{m} m^{-5 / 2}\left(r^{2} p\right)^{m}\left(r^{2} p\right)^{-1} e^{-p m(n-m)},
$$

que por sua vez é igual a

$$
\frac{N^{m}}{r^{2} p} m^{-5 / 2} \exp \left\{m \ln \left(2 e r^{2}\right)+m(\ln p-p(n-m))\right\} .
$$

Agora, vamos analisar o ultimo termo da equação para podermos prosseguir com as desigualdades. Substituindo $p$ definido em (4.5) temos que o termo $m(\ln p-p(n-m))$ é igual a

$$
m\left(\ln \left(\frac{\ln N+c(N)}{n}\right)-\left(\frac{\ln N+c(N)}{n}\right)(n-m)\right) .
$$

Que pode ser escrito como

$$
m\left[\ln (\ln N+c(N))-\ln n-(\ln (N)+c(N))+(\ln (N)+c(N)) \frac{m}{n}\right],
$$

sendo igual a

$$
m\left[\ln (\ln N+c(N))-(\ln N+c(N))\left(1-\frac{m}{n}\right)\right]-m \ln (n)
$$

Como $m \leq N / 2 \leq n / 2$, então a expressão anterior é menor que

$$
m\left[\ln (\ln N+c(N))-(\ln N+c(N))\left(1-\frac{n / 2}{n}\right)\right]-m \ln (n),
$$

que é igual a

$$
m\left[\ln (\ln N+c(N))-\frac{\ln N+c(N)}{2}\right]-m \ln (n) .
$$

Agora, observe que para um $k>0$ arbitrário, existe um $x^{\prime}>0$ tal que para todo $x>x^{\prime}$, temos que $\ln (x)<\frac{x}{k}$. Usando $x=(\ln N+c(N))$, podemos limitar a expressão (4.27) por

$$
m\left[\left(\frac{\ln N+c(N)}{k}\right)-\frac{\ln N+c(N)}{2}\right]-m \ln (n),
$$

que pode ser reescrito como

$$
m\left[(\ln N+c(N))\left(\frac{1}{k}-\frac{1}{2}\right)\right]-m \ln (n),
$$

para $N$ suficientemente grande.

Sem perca de generalidade, tomamos $k>2$. Assim, usando a expressão anterior e retomando as desigualdades iniciais, podemos limitar (4.26) por

$$
\left(\frac{N}{n}\right)^{m} \frac{m^{-5 / 2}}{r^{2} p} \exp \left\{m\left[\left(\frac{2-k}{2 k}\right)(\ln (N)+c(N))+\ln \left(2 e r^{2}\right)\right]\right\},
$$


que é igual a

$$
\left(\frac{N}{n}\right)^{m} \frac{m^{-5 / 2}}{r^{2} p} \exp \left\{-m\left[\left(\frac{k-2}{2 k}\right)(\ln (N)+c(N))-\ln \left(2 e r^{2}\right)\right]\right\}
$$

Veja agora que, como $n>N$, $\operatorname{logo}\left(\frac{N}{n}\right)^{m}<1$. Por simplicidade, consideramos $c(N)=$ $c>0$ obtendo que $-m\left(\frac{k-2}{2 k}\right) c(N)<0$. O fato onde $c(N)=c \leq 0$ leva aos mesmos resultados porém é necessário pequenas modificações na prova, que podem ser vistas Kang, Pachón e Rodríguez (2016). Continuando, temos que

$$
\left(\frac{N}{n}\right)^{m} \exp \left\{-m\left(\frac{k-2}{2 k}\right) c(N)\right\}<1
$$

Então, a probabilidade de existir um componente de tamanho $m$ é menor que

$$
\frac{m^{-5 / 2}}{r^{2} p} \exp \left\{-m\left[\left(\frac{k-2}{2 k}\right) \ln (N)-\ln \left(2 e r^{2}\right)\right]\right\} \text {. }
$$

Podemos reescrever a expressão anterior como

$$
\frac{m^{-5 / 2}}{r^{2} p} \exp \left\{\ln (N)^{-m \frac{(k-2)}{2 k}}+\ln \left(2 e r^{2}\right)^{m}\right\}
$$

que por sua vez é igual a

$$
\frac{m^{-5 / 2}\left(2 e r^{2}\right)^{m}}{r^{2} p N^{\frac{m(k-2)}{2 k}}}
$$

Substituindo $p$ como definido em (4.5) teremos (4.28) igual a

$$
\frac{n m^{-5 / 2}\left(2 e r^{2}\right)^{m}}{r^{2}(\ln (N)+c(N)) N^{\frac{m(k-2)}{2 k}}} \text {. }
$$

Analisando $n$, vemos que, como $r$ é o tamanho do maior super-vértice, podemos obter a desigualdade $N \leq n \leq N r$. Logo a expressão anterior é menor que

$$
\frac{N r m^{-5 / 2}\left(2 e r^{2}\right)^{m}}{r^{2}(\ln (N)+c(N)) N^{\frac{m(k-2)}{2 k}}}=\frac{m^{-5 / 2}\left(2 e r^{2}\right)^{m}}{r(\ln (N)+c(N)) N^{\frac{m(k-2)}{2 k}}-1} .
$$

Agora, vamos separar em dois casos. No primeiro considere $3 \leq m \leq N / 2$. Assim, basta tomarmos $\mathrm{k}>6$ para obtermos uma convergência para 0 no limite onde $N \rightarrow \infty$. Sem perca de generalidade, tomamos $k=7$, e assim a expressão (4.29) anterior é igual a

$$
\frac{\left(2 e r^{2}\right)^{m}}{m^{5 / 2} r(\ln (N)+c(N)) N^{\frac{5 m-14}{14}}} .
$$

Pela nossa hipótese, $c_{*}=\infty$ ou $c_{*}=c$, assim tomamos $c(N)=c$, e como $m \leq N / 2$, então $2 m \leq N$. Por consequência, a expressão (4.30) é menor que

$$
\frac{\left(2 e r^{2}\right)^{m}}{m^{5 / 2} r(\ln (N)+c)(2 m)^{\frac{5 m-14}{14}}} .
$$


Conseguimos assim a seguinte inequação:

$$
\mathbb{P}(\exists \text { um componente de tamanho } m)<\frac{\left(2 e r^{2}\right)^{m}}{m^{5 / 2} r(\ln (N)+c)(2 m)^{\frac{5 m-14}{14}}} .
$$

Aplicando o limite e o somatório teremos

$$
\lim _{N \rightarrow \infty} \sum_{m=3}^{N / 2} \mathbb{P}(\exists \text { um componente de tamanho } m)<\lim _{N \rightarrow \infty} \sum_{m=3}^{N / 2} \frac{\left(2 e r^{2}\right)^{m}}{m^{5 / 2}(2 m)^{\frac{5 m-14}{14}}} \frac{1}{r(\ln (N)+c)}
$$

onde o lado direito da inequação anterior pode ser escrito como

$$
\sum_{m=3}^{\infty} \frac{\left(2 e r^{2}\right)^{m}}{m^{5 / 2}(2 m)^{\frac{5 m-14}{14}}}\left(\lim _{N \rightarrow \infty} \frac{1}{r(\ln (N)+c)}\right) \text {. }
$$

Devido ao fato de que

$$
\lim _{m \rightarrow \infty} \frac{2 e r^{2}}{m^{\frac{5}{2 m}}(2 m)^{\frac{5 m-14}{14 m}}}=0<1
$$

Temos pelo teste de Cauchy (teste da raíz) que a série em (4.31) converge, e como $\lim _{N \rightarrow \infty} \frac{1}{r\left(\ln (N)+c_{1}\right)}=0$, temos que

$$
\lim _{N \rightarrow \infty} \sum_{m=3}^{N / 2} \mathbb{P}(\exists \text { um componente de tamanho } m)=0 .
$$

Agora, nos falta verificar qual o comportamento para o segundo caso onde $m=2$. Começamos os cálculos a partir da expressão (4.25). Assim, temos

$$
\mathbb{P}(\exists \text { um componente de tamanho } 2)<\left(\begin{array}{l}
N \\
2
\end{array}\right) 2^{2-2}\left(r^{2} p\right)^{(2-1)}(1-p)^{2(n-2)} .
$$

Definindo $p$ como em (4.5) e utilizando novamente de que $1-p<e^{-p}$ temos que o lado direito na inequação é menor que

$$
\left(\begin{array}{l}
N \\
2
\end{array}\right) \frac{r^{2}(\ln (N)+c(N))}{n} e^{-2 p(n-2)}
$$

Vendo que

$$
\frac{N !}{(N-2) !} \frac{1}{n} \leq \frac{N(N-1)}{N}<N
$$

temos que (4.33) é menor que

$$
\frac{N r^{2}(\ln (N)+c(N))}{2 e^{2 p(n-2)}}
$$


Analisando $e^{2 p(n-2)}$ temos

$$
\begin{aligned}
e^{2 p(n-2)} & =\exp \left\{\frac{2(\ln (N)+c(N))(n-2)}{n}\right\} \\
& =\exp \left\{(\ln (N)+c(N))\left(2-\frac{4}{n}\right)\right\} \\
& =\exp \left\{\ln (N)\left(\frac{2 n-4}{n}\right)+c(N)\left(\frac{2 n-4}{n}\right)\right\} \\
& =N^{\frac{2 n-4}{n}} e^{c(N)\left(\frac{2 n-4}{n}\right)} .
\end{aligned}
$$

Logo,

$$
\frac{N r^{2}(\ln (N)+c(N))}{2 e^{2 p(n-2)}}=\frac{N r^{2}(\ln (N)+c(N))}{2 N^{\frac{2 n-4}{n}} e^{c(N)\left(\frac{2 n-4}{n}\right)}}=\frac{r^{2}(\ln (N)+c(N))}{2 N^{\frac{n-4}{n}} e^{c(N)\left(\frac{2 n-4}{n}\right)}} .
$$

Agora, veja que $\frac{2 n-4}{n} \geq 1$ para $n$ suficientemente grande, mais especificamente para $n \geq 4$. Então $e^{c(N)\left(\frac{2 n-4}{n}\right)}>c(N)$ quando $N \rightarrow \infty$. Logo

$\lim _{N \rightarrow \infty} \mathbb{P}\left(\exists\right.$ um componente de tamanho 2) $<\lim _{N \rightarrow \infty} \frac{r^{2}(\ln (N)+c(N))}{2 N^{\frac{n-4}{n}} c(N)}=0$.

E finalmente, por (4.32) e (4.34) temos que

$$
\lim _{N \rightarrow \infty} \sum_{m=2}^{N / 2} \mathbb{P}(\exists \text { um componente de tamanho } m)=0 .
$$

\subsection{Prova do Teorema de Conectividade}

Vamos iniciar a prova definindo o evento $A=\{\mathrm{O}$ grafo $G(N, \mathcal{K}, p)$ é desconectado $\}$. Temos assim, que o evento $\{X \geq 1\} \subset A$, pois havendo ao menos um super-vértice isolado, o grafo já irá ser desconectado. Logo

$$
\begin{aligned}
\mathbb{P}(X \geq 1) & \leq \mathbb{P}(A) \\
1-\mathbb{P}(X=0) & \leq \mathbb{P}(A) .
\end{aligned}
$$

Para as condições do item 1 do Teorema 4.2.1, o Lema 2 nos diz que $\lim _{N \rightarrow \infty} \mathbb{P}(X=$ $0)=0$

Fazendo com que

$$
\lim _{N \rightarrow \infty}(1-\mathbb{P}(X=0))=1,
$$

tendo assim, pela expressão (4.35), que

$$
\lim _{N \rightarrow \infty} \mathbb{P}(A)=1 .
$$


Com isso, pelo complementar de $A$, provamos a primeira afirmação do Teorema 4.2.1, onde nos diz que, para $c_{*}=-\infty$, temos que $\lim _{N \rightarrow \infty} \mathbb{P}[G(N, \mathcal{K}, p)$ ser conectado $]=0$.

Agora, sejam $C_{1}=\{\mathrm{O}$ grafo $G(N, \mathcal{K}, p)$ possui pelo menos um super-vértices isolado $\}=$ $\{X \geq 1\}$, e $C_{2}=\{\mathrm{O}$ grafo $G(N, \mathcal{K}, p)$ possui componentes de tamanho $m$, com $2 \leq m \leq N / 2\}$. Podemos escrever

$$
\begin{aligned}
A & =C_{1} \cup C_{2} \\
\mathbb{P}(A) & =\mathbb{P}\left(C_{1}\right)+\mathbb{P}\left(C_{2}\right)-\mathbb{P}\left(C_{1} \cap C_{2}\right) \\
-\mathbb{P}(A) & =-\mathbb{P}\left(C_{1}\right)-\mathbb{P}\left(C_{2}\right)+\mathbb{P}\left(C_{1} \cap C_{2}\right) \\
1-\mathbb{P}(A) & =1-\mathbb{P}\left(C_{1}\right)-\mathbb{P}\left(C_{2}\right)+\mathbb{P}\left(C_{1} \cap C_{2}\right) \\
\mathbb{P}\left(A^{c}\right) & =\mathbb{P}\left(C_{1}^{c}\right)-\mathbb{P}\left(C_{2}\right)+\mathbb{P}\left(C_{1} \cap C_{2}\right) \\
\mathbb{P}\left(A^{c}\right) & =\mathbb{P}(X=0)-\mathbb{P}\left(C_{2}\right)+\mathbb{P}\left(C_{1} \cap C_{2}\right) .
\end{aligned}
$$

Pelo Lema 3 vimos que nas condições do item 2 do Teorema 4.2.1, $\lim _{N \rightarrow \infty} \mathbb{P}(X=$ $0)=e^{-\mu_{1} e^{-c}}$. E pelo Lema 4 podemos afirmar que $\lim _{N \rightarrow \infty} \mathbb{P}\left(C_{2}\right)=0$, fazendo com que $\lim _{N \rightarrow \infty} \mathbb{P}\left(C_{1} \cap C_{2}\right)=0$. Com isso obtemos que

$$
\lim _{N \rightarrow \infty} \mathbb{P}\left(A^{c}\right)=e^{-\mu_{1} e^{-c}}
$$

Provando assim o item 2 do Teorema 4.2.1.

Temos pelo Lema 2 que, sob as condições do item 3 do Teorema $4.2 .1, \lim _{N \rightarrow \infty} \mathbb{P}(X=$ $0)=1$, e novamente pelo Lema 4 , podemos afirmar que $\lim _{N \rightarrow \infty} \mathbb{P}\left(C_{2}\right)=0$ e $\lim _{N \rightarrow \infty} \mathbb{P}\left(C_{1} \cap\right.$ $\left.C_{2}\right)=0$. Utilizando novamente da expressão (4.36) temos que

$$
\lim _{N \rightarrow \infty} \mathbb{P}\left(A^{c}\right)=1
$$

ou seja, o grafo $G(N, \mathcal{K}, p)$ é conectado com alta probabilidade, provando o item 3 do Teorema 4.2.1.

Tendo os três itens provados, conseguimos demonstrar o Teorema 4.2.1 por completo. 


\section{SIMULAÇÕES E RESULTADOS}

Os grafos aleatórios são geralmente utilizados para modelar redes complexas, no intuito de compreender certas propriedades e comportamentos. Estas redes possuem alto grau de complexidade e dinâmica, onde geralmente é impossível descrever sua topologia sem a utilização de medidas específicas.

Neste capítulo, vamos realizar algumas simulações no propósito de representar duas redes reais, verificando os pontos positivos e negativos do método por meio de informações quantitativas sobre a eficiência destas simulações, que obtemos utilizando-se de medidas e métodos que denominamos por Medidas Topológicas (veja Apêndice C).

\subsection{Métodos de simulação}

Foram desenvolvidos três códigos diferentes para simular o grafo aleatório $G(N, \mathcal{K}, p)$. Ambas utilizando o software $\mathrm{R}$ (R Core Team, 2013) e o pacote igraph (CSARDI; NEPUSZ, 2006) desenvolvido para o estudo de grafos.

- No primeiro método de construção usamos de um grafo binomial $G(n, p)$ já formado, e com ele estabelecemos os super-vértices utilizando de um vetor $z=\left\{z_{1}, z_{2}, \ldots, z_{n}\right\}$, onde cada elemento de $z$ é a etiqueta que designa ao qual super-vértice o vértice de $G(n, p)$ pertence. Sendo assim, $z$ tem comprimento $n$, necessariamente. O maior valor de $z$ será a quantia $N$ de super-vértices de $G(N, \mathcal{K}, p)$. O procedimento consiste em criar um grafo nulo $G_{2}$ com $N$ vértices e conectá-los de acordo com as conexões em $G(n, p)$. Para isso, o código necessita varrer todos os vértices em $G(n, p)$ verificando quais são seus vizinhos e à quais super-vértices eles pertencem, após a verificação do vértice suas arestas são apagadas para agilizar o processo. Note que este processo pode ser um tanto demorado devido a grande quantia de vértices que $G(n, p)$ pode possuir, isto é, seu custo computacional está relacionada com a quantia $n$. Para criar 
o grafo $G(n, p)$ inicial basta usar a função erdos.renyi.game(n,p,type="gnp") no software R. Obs: Note que este código permite a construção do grafo com supervértices utilizando de um grafo inicial qualquer, não sendo necessariamente um grafo aleatório binomial.

- O segundo método é uma construção direta, utilizando do conhecimento a respeito da probabilidade $p$. Neste caso não é necessário criar o grafo $G(n, p)$ inicial, basta estabelecer $p$ e um vetor $K=\left\{K_{1}, K_{2}, \ldots, K_{N}\right\}$ contendo o tamanho de cada supervértice. Assim $N$ é automaticamente estabelecido como sendo o comprimento de $K$. Note que $K \neq z$. Com isso, o código estabelece os tamanhos e conecta cada supervértice utilizando da probabilidade $p_{i j}=1-(1-p)^{i j}$ apresentada na Equação (4.1). O custo computacional deste processo esta relacionado com a quantia $N$, pois é necessário a verificação, de estabelecer uma aresta ou não, para todos os possíveis pares diferentes de super-vértices, ou seja, é preciso $\left(\begin{array}{c}N \\ 2\end{array}\right)=\frac{N(N-1)}{2}$ verificações.

- O terceiro método também é uma construção direta, mas agora utilizando do conhecimento a respeito de $|E(G(N, \mathcal{K}, p))|=: \mathcal{M}$. Neste caso nós estabelecemos $\mathcal{M} \in \mathbb{N}$, (número de arestas entre os super-vértices), e novamente $K$ sendo o vetor contendo os tamanhos de cada super-vértice. Da mesma forma, $N$ será o comprimento do vetor $K$. O código irá formar os super-vértices com a configuração do vetor $K$ e incluir as $\mathcal{M}$ arestas da seguinte forma: a cada período de tempo, dois super-vértices diferentes são escolhidos independentemente, cada um com probabilidade $i / n$ ( $i$ sendo o tamanho do super-vértice), então é incluso uma aresta entre estes dois super-vértices caso eles não sejam conectados, o processo finaliza até incluir $\mathcal{M}$ arestas. Desta vez, o custo computacional é relacionado a quantia $\mathcal{M}$.

Os três códigos podem ser visto no Anexo A.

\subsection{Simulações para duas redes reais}

Iremos usar do modelo para simular duas redes reais fornecidas pelo site KONECT (KUNEGIS, 2013).

\section{Rede Proteína}

Vamos denominar a primeira rede real estudada como Proteína. Ela é composta por vértices que representam proteínas contidas na levedura, e arestas representando uma interação metabólica entre duas proteínas. O Arquivo com a constituição do grafo pode ser baixado no link < http://konect.uni-koblenz.de/networks/moreno_propro>.

Para simular esta rede reproduzimos dez grafos aleatórios com o segundo método apresentado, isto é, utilizando da forma direta com ligações a partir da probabilidade $p$. 
Nosso objetivo era de obter simulações com a distribuição de graus o mais semelhante possível com a rede real, juntamente com a quantia de vértices e arestas. Assim, a escolha dos tamanhos para os super-vértices foi determinada pela quantia de graus de cada vértice na rede real. Como alguns vértices da rede real possuem grau 0 tivemos de acrescentar 1 , ou seja, empregamos $K_{i}=d\left(v_{i}\right)+1 ; v_{i} \in G_{\text {pro }}$, onde $G_{\text {pro }}$ é o grafo que representa a rede Proteína, caso isto não fosse feito, muitos super-vértices teriam tamanho 0, ou seja, não iriam existir, fazendo com que o número de vértices da rede real fosse diferente do número de super-vértices da simulação. A escolha de igualar o tamanho dos super-vértices do modelo com o grau dos vértices na rede real se torna razoável, pelo fato de ser possível relacionar o tamanho com o grau médio em um super-vértice. Note que automaticamente, o tamanho do vetor $K$ será igual a quantia de vértices da rede Proteína, resolvendo o problema para a quantia de vértices. Uma pergunta importante surge neste momento. Qual o valor apropriado de $p$ para obtermos uma simulação com a quantia de arestas próximo ao valor da rede real? Para esta pergunta, desenvolvemos o seguinte pensamento.

Seja $M=|E(G)| \operatorname{com} G=G(n, p)$. Temos por (2.1) que a média de arestas no grafo binomial é dado por

$$
\mathbb{E}\left[Y_{E}\right]=p\left(\begin{array}{l}
n \\
2
\end{array}\right) .
$$

Logo, como queremos que $Y_{E}=M$, podemos obter

$$
p \approx \frac{2 M}{n(n-1)}
$$

Note que, caso o grafo $G(N, \mathcal{K}, p)$ possuísse super-vértices de mesmo tamanho, a igualdade anterior também seria satisfeita mas, da seguinte forma

$$
p_{i i} \approx \frac{2 \mathcal{N}}{N(N-1)} .
$$

Como os tamanhos dos super-vértices se diferem, este fato não acontece. Assim, tomamos a aproximação

$$
P_{r}:=\sum_{i, j=1}^{r} \frac{p_{i j}}{Z} \approx \frac{2 \mathcal{M}}{N(N-1)},
$$

onde $Z$ são todas as combinações possíveis entre os tamanhos de super-vértices existentes.

$$
\begin{aligned}
& \text { Como } p_{i j}=1-(1-p)^{i j} \text { temos que } \\
& \qquad p=1-\left(1-p_{i j}\right)^{\frac{1}{i j}} .
\end{aligned}
$$

Devido a aleatoriedade dos tamanhos dos super-vértices vamos utilizar de uma medida que possa representar estes valores satisfatoriamente. Em especial, para a rede que queremos simular, os tamanhos dos super-vértices são iguais aos graus dos vértices na rede, acrescentado de 1. A média dos tamanhos resulta em 3,35615 com desvio-padrão de 3, 123874 . Devido a este fato, para obtermos um $p$ fixo, vamos tomar

$$
p=1-\left(1-P_{r}\right)^{\frac{1}{\left(\sum_{i=1}^{r} \frac{i}{N}\right)^{2}}} .
$$


Neste caso, para simular a rede Proteína, tomamos $\mathcal{M}=M=2203, N=1870$ e obtemos

$$
\begin{gathered}
P_{r}=0,001260647 \\
p=1-(1-0,001260647)^{\frac{1}{(3,35615)^{2}}}=0,0001119852 .
\end{gathered}
$$

Posteriormente vamos ver que este valor de $p$ realmente nos proporciona um número de arestas próximo ao da rede real Proteína, satisfazendo nosso objetivo. Entretanto, o valor obtido nos fornece bons resultados devido ao baixo desvio padrão que os graus da rede possuem. Caso fossemos utilizar deste mesmo pensamento para encontrar um valor para $p$, pretendendo usar o segundo método para simular a segunda rede real que iremos apresentar, a quantia de arestas não seria tão satisfatória, pois o desvio padrão dos graus da segunda rede é significativamente maior, sendo que a média dos tamanhos não seria uma boa medida para substituir $i j$ por $\left(\sum_{i=1}^{r} \frac{i}{N}\right)^{2}$.

Com $p$ definido, nós geramos dez simulações representando a rede real. Com isso, apresentamos na Tabela 1 as medidas topológicas calculadas para a rede real e as médias e desvios-padrão destas medidas para as dez simulações geradas.

Tabela 1 - Valores de Medidas Topológicas

\begin{tabular}{|c|c|c|c|}
\hline Medidas & Rede Proteína & Média das simulações & Desvios padrão das simulações \\
\hline \hline$|V(G)|$ & 1870 & $\mathbf{1 8 7 0}$ & 0 \\
\hline$|E(G)|$ & 2203 & $\mathbf{2 2 4 0 , 2}$ & 67,15951161 \\
\hline $\mathrm{n}^{\mathbf{O}}$ comp. & 173 & 349,10 & 11,9485006 \\
\hline $\max \left(d\left(v_{i}\right)\right)$ & 56 & 41,5 & 5,338539126 \\
\hline $\mathbb{E}\left[\boldsymbol{D}_{\boldsymbol{v}}\right]$ & 2,35615 & $\mathbf{2 , 3 9 5 9 3 5 8}$ & 0,071828365 \\
\hline$\tilde{H}$ & 0,1989755 & 0,22342908 & 0,004461585 \\
\hline$A S$ & $-0,1615299$ & 0,002051081 & 0,028094015 \\
\hline $\mathbb{E}[\boldsymbol{C}]$ & 0,1529893 & 0,004196451 & 0,001992837 \\
\hline $\boldsymbol{C}_{\Delta}$ & 0,0550095 & 0,00468525 & 0,001201253 \\
\hline$E$ & 0,09973485 & 0,11391093 & 0,004530068 \\
\hline $\mathrm{Diam}$ & 19 & 15,3 & 1,418136492 \\
\hline $\mathbb{E}[\boldsymbol{B}]$ & 3301,518 & 2918,5172 & 67,63600889 \\
\hline
\end{tabular}

Fonte: Dados da pesquisa.

Na Figura 19, mostramos a densidade da distribuição dos graus em conjunto com a densidade da média dos graus das simulações. Na Figura 21 mostramos a densidade acumulada dos coeficientes de aglomeração locais para a rede real e para as simualções. $\mathrm{Na}$ Figura 22 mostramos as densidades para as distribuições dos betweenness. Já na Figura 23 é apresentado os gráficos $k \times k_{n n}$ para a rede Proteína e para as três primeiras simulações.

Devido aos dados obtidos podemos ver que o objetivo de assemelhar a distribuição dos graus nas simulações é satisfeito. Pela tabela vemos que a média dos graus e a quantia de arestas possuem um desvio padrão que inclui as quantias apresentadas na rede real. 


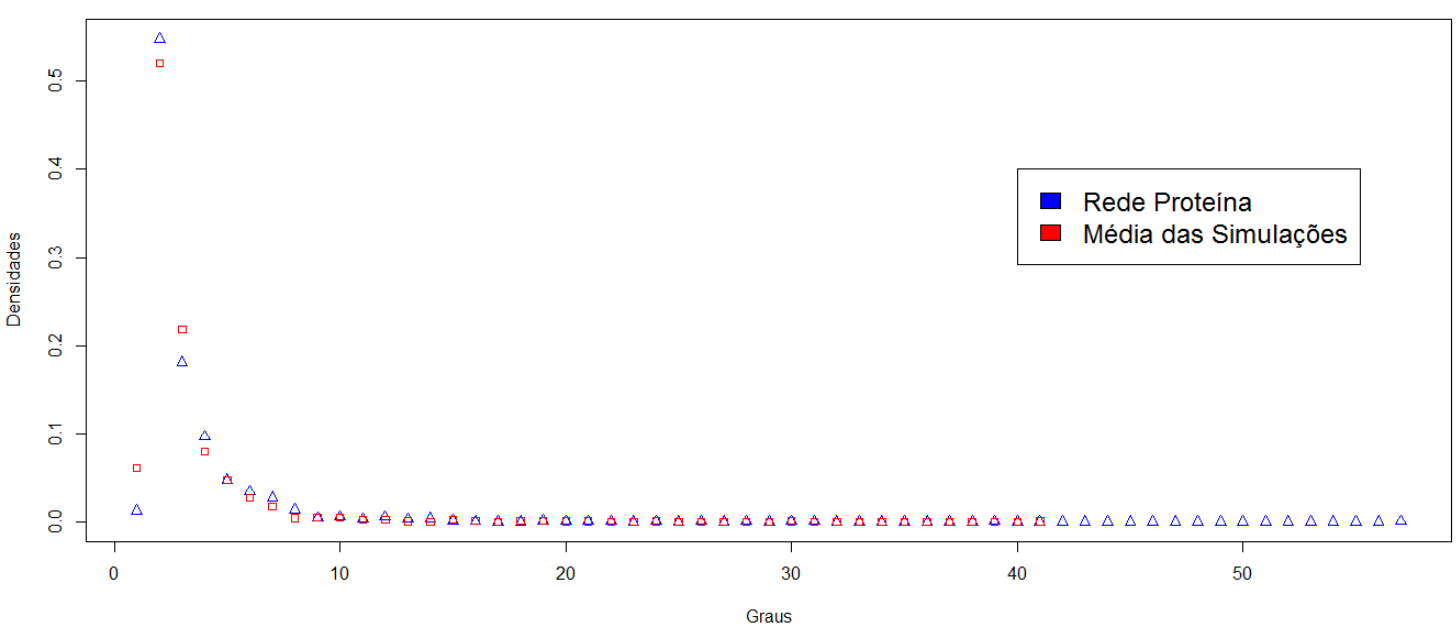

Figura 19 - Distribuições dos graus

Fonte: Dados da pesquisa.

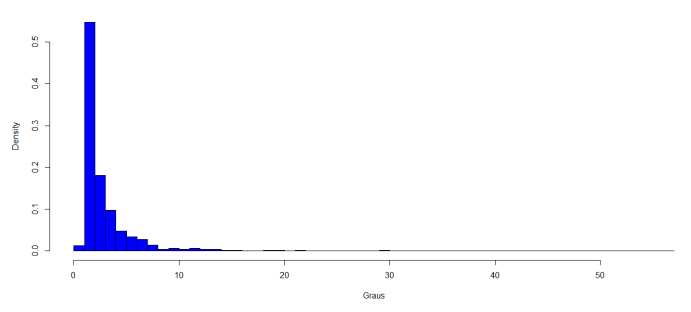

(a) Proteína

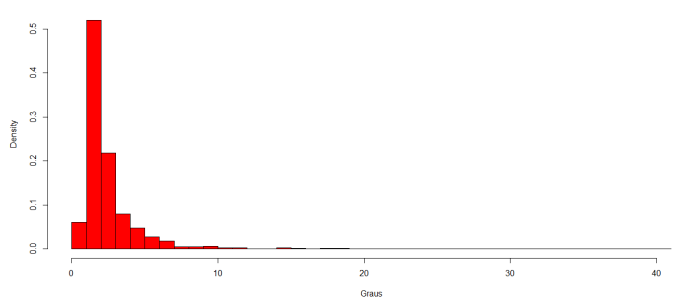

(b) Média das Simulações

Figura 20 - Distribuições dos graus

Fonte: Dados da pesquisa.

O gráfico para a distribuição dos graus mostra uma semelhança muito grande em suas densidades, como era o nosso propósito. Entretanto, em relação a aglomeração, tando os dados da tabela quanto os dados apresentados no gráfico dos coeficientes mostram uma diferença considerável. A média do coeficientes de aglomeração local e a transitividade estão distantes com o encontrado na rede real, e o gráfico nos indica que existem muito mais coeficientes com níveis altos na rede real do que nas simulações. Em relação a centralidade, pela análise do gráfico dos betweenness, vemos que a distribuição é a mesma entre a rede real e as simulações. Isto nos diz que a quantia de pontos importantes na rede é a mesma das simulações, um ponto positivo para o modelo. A eficiência e o diâmetro se diferem um pouco pois a aglomeração diferente acaba influenciando nestes valores. Por fim, vemos que a rede real possui um coeficiente de assortatividade levemente negativo, ou seja, negativo e próximo a zero. As simulações não conseguiram se aproximar deste coeficiente mas também não resultaram em valores relativamente distantes. 


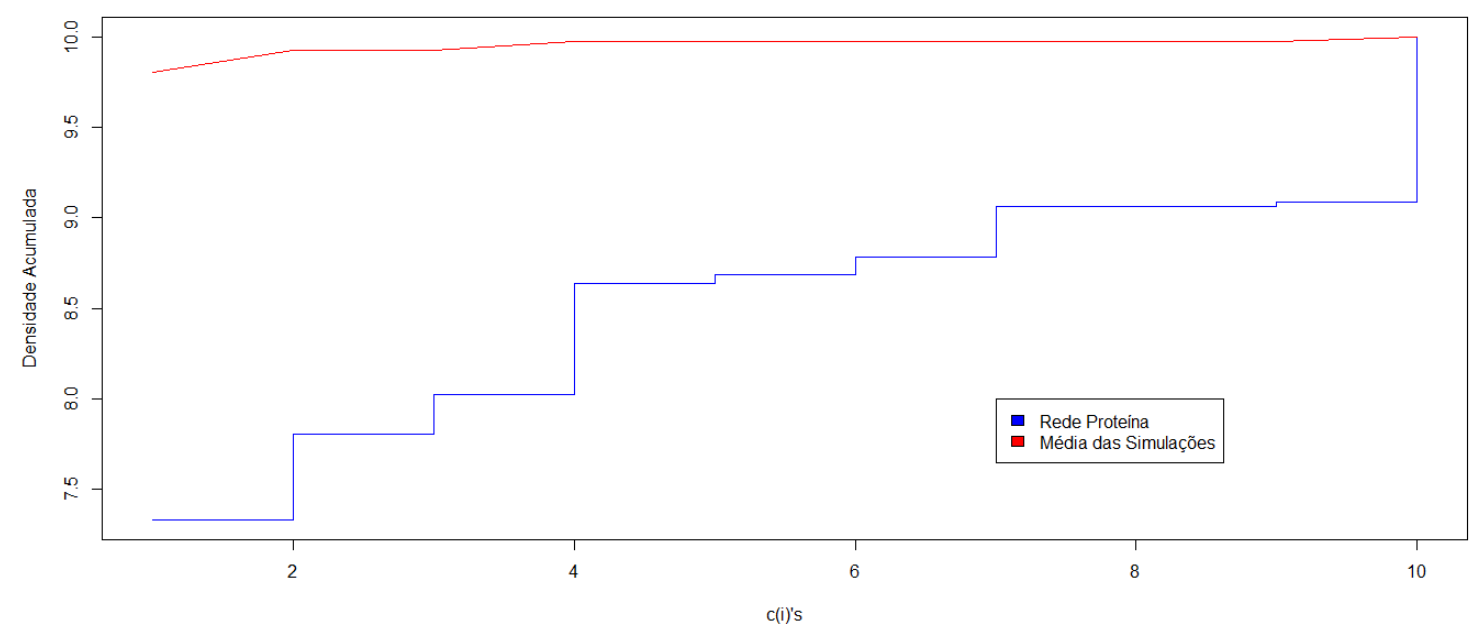

Figura 21 - Distribuição acumulada dos coeficientes de aglomeração locais

Fonte: Dados da pesquisa.

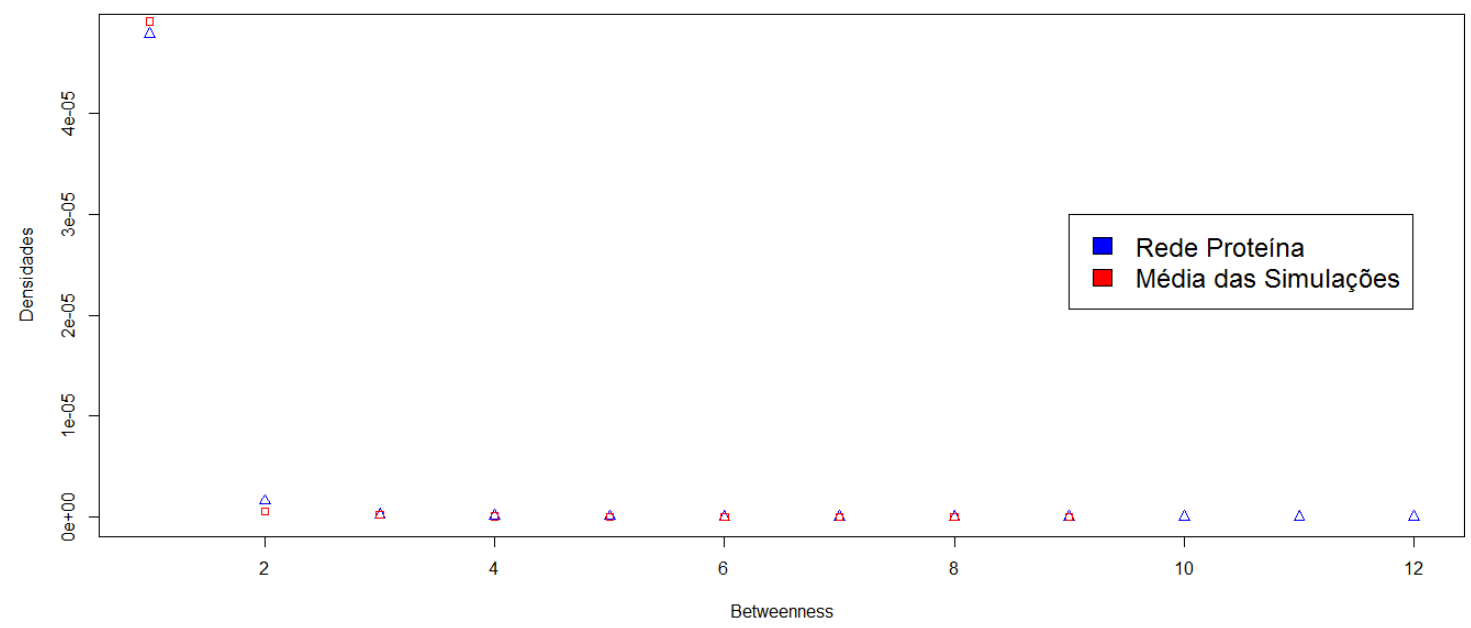

Figura 22 - Distribuições dos Betweenness

Fonte: Dados da pesquisa.

\section{Rede Facebook}

Denominamos a próxima rede real estudada como Facebook, pois ela é composta de vértices que representam usuários da rede social Facebook (SCHROEDER, 2012), e suas arestas representam a relação de amizade por dois usuários da rede. O arquivo com a constituição do grafo pode ser obtido em < http://konect.uni-koblenz.de/networks/ ego-facebook $>$.

Neste caso criamos dez simulações mas, utilizando do terceiro método apresentado, isto é, pela forma direta usando uma quantia $\mathcal{M}$ de arestas. Obviamente tomamos $\mathcal{M}$ igual 


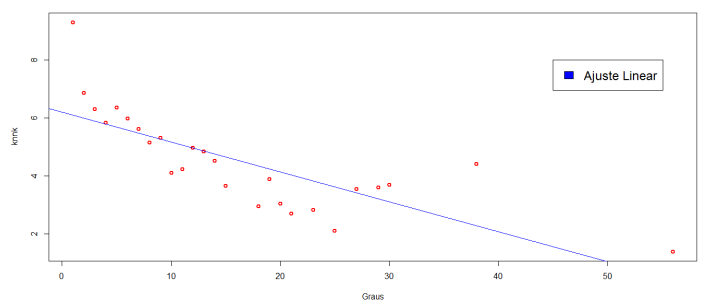

(a) Proteína

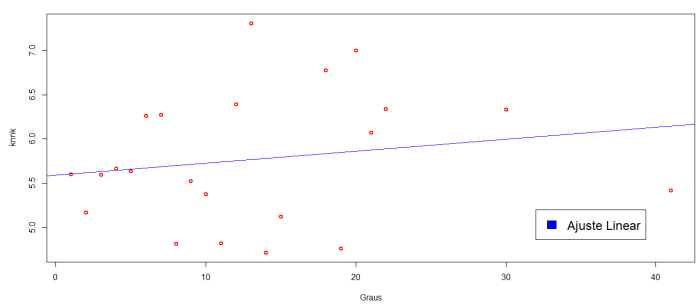

(c) $P_{2}$

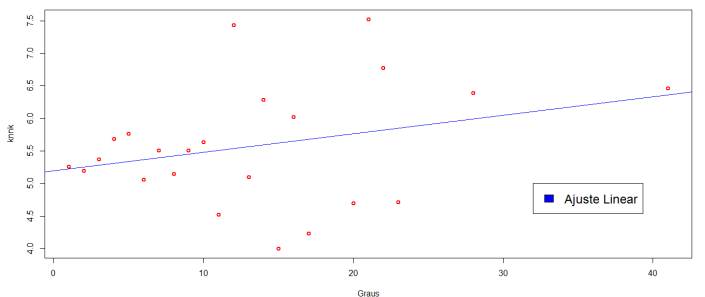

(b) $P_{1}$

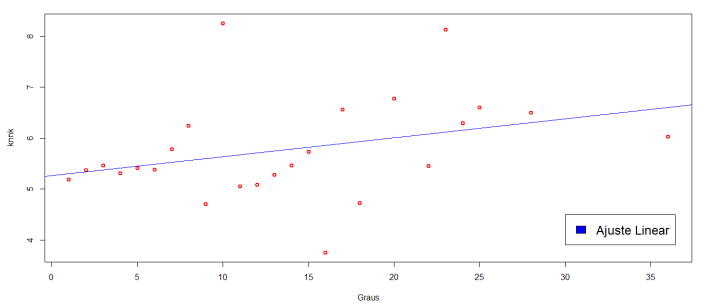

(d) $P_{3}$

Figura 23 - Graficos $k \times k_{n n}$

Fonte: Dados da pesquisa.

ao número de arestas da rede real e o vetor de tamanhos dos super-vértices igual aos graus dos vértices da rede. Desta vez não foi preciso o acréscimo de 1 nos tamanhos dos supervértices pois a rede real não apresentava nenhum vértice isolado. Note que este método nos da maior controle da quantia de arestas na simulação e consequentemente, do grau médio.

Os resultados das medidas topológicas para a rede real Facebook e as médias e desvios padrão das simulações são apresentadas na Tabela 2.

Tabela 2 - Valores de Medidas Topológicas

\begin{tabular}{|c|c|c|c|}
\hline Medidas & Rede Facebook & Médias das simulações & Desvios padrão das simulações \\
\hline \hline$|V(G)|$ & 2888 & $\mathbf{2 8 8 8}$ & 0 \\
\hline$|E(G)|$ & 2981 & $\mathbf{2 9 8 1}$ & 0 \\
\hline $\mathrm{n}^{\mathrm{o}}$ comp. & 1 & 806,2 & 13,65283853 \\
\hline $\max \left(d\left(v_{i}\right)\right)$ & 769 & 522,4 & 21,38119631 \\
\hline $\mathbb{E}\left[\boldsymbol{D}_{\boldsymbol{v}}\right]$ & 2,064404 & $\mathbf{2 , 0 6 4 4 0 4}$ & 0 \\
\hline$\tilde{H}$ & 0,02213017 & 0,1378524 & 0,000759678 \\
\hline$A S$ & $-0,668214$ & $-0,35921015$ & 0,007946481 \\
\hline $\mathbb{E}[\boldsymbol{C}]$ & 0,8029648 & 0,29748685 & 0,015593546 \\
\hline$C_{\Delta}$ & 0,00035938 & 0,006644099 & 0,000267396 \\
\hline$E$ & 0,2934442 & 0,15591523 & 0,002908411 \\
\hline $\mathrm{Diam}$ & 9 & 11,6 & 0,516397779 \\
\hline $\mathbb{E}[\boldsymbol{B}]$ & 4139,123 & 1620,2373 & 47,00731939 \\
\hline
\end{tabular}

Fonte: Dados da pesquisa. 
Da mesma forma feita para a primeira rede real, as distribuições de probabilidade em relação a rede e as médias das simulações são apresentadas nas Figura 24 à Figura 27. Os gráficos $k \times k_{n n}$ são mostrados na Figura 28 .

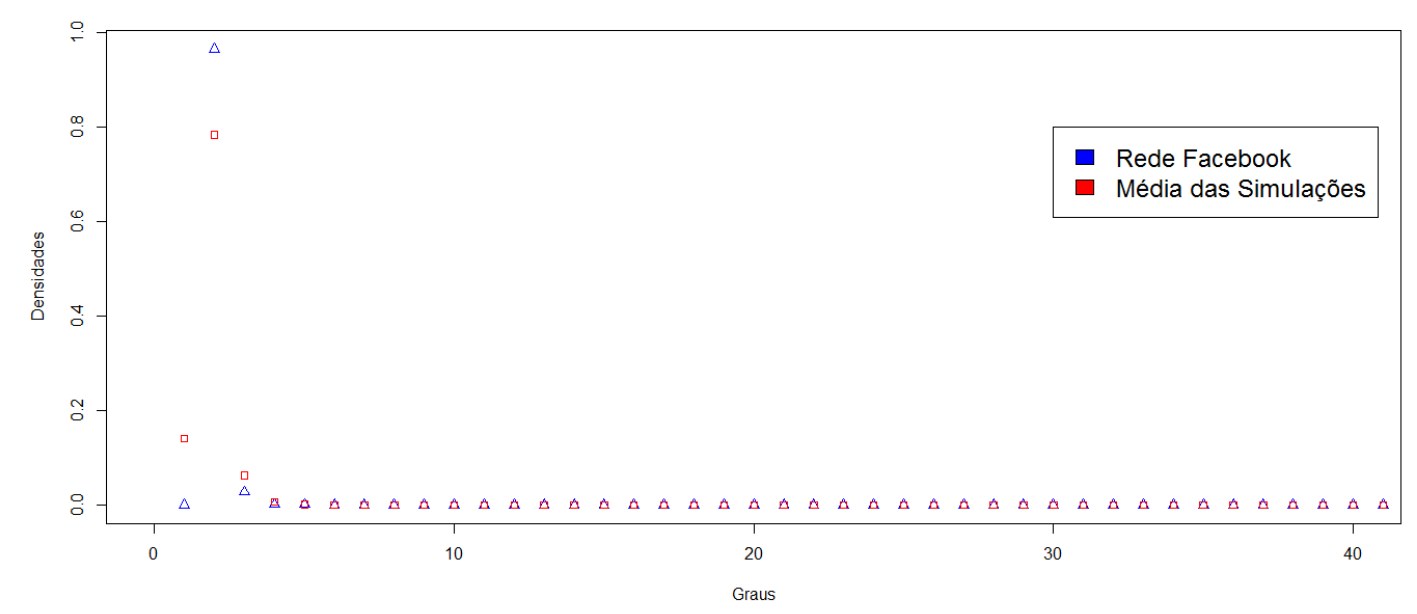

Figura 24 - Distribuições dos graus

Fonte: Dados da pesquisa.

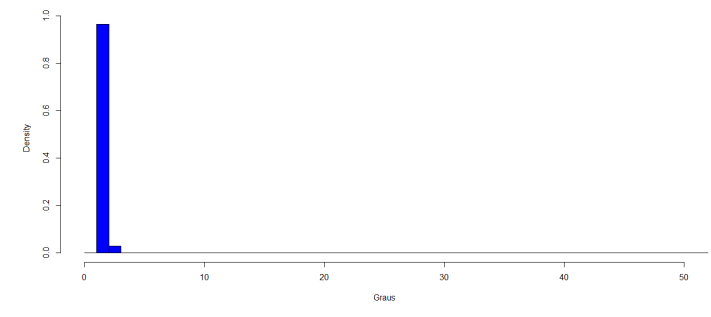

(a) Facebook

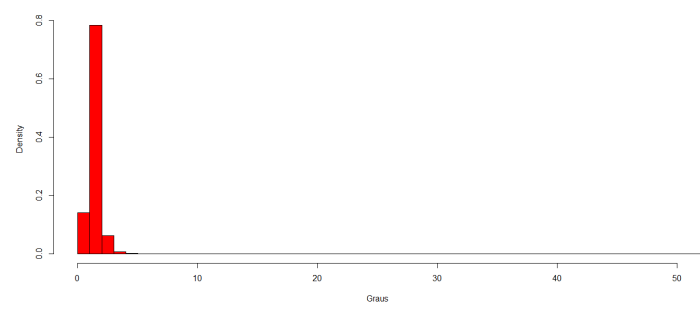

(b) Média das Simulações

Figura 25 - Distribuições dos graus

Fonte: Dados da pesquisa.

O resultados nos mostram novamente que a distribuição dos graus é compatível com a da rede real. Com este método de simulação conseguimos estabelecer o número de vértices, arestas e consequentemente o grau médio, de forma idêntica ao da rede real. Novamente as medidas relacionadas a aglomeração se diferem da rede real. O interessante neste caso foram os resultados em relação a centralidade. Os gráficos dos coeficientes betweenness possuem distribuição semelhante, porém as proporções de importância não são. Ocasionando uma grande diferença entre a média dos betweenness entre a rede real e as simulações, como pode ser visto na Tabela 2. Em relação a assortatividade, as simulações obtiveram resultados similares a da rede real.

Para esta rede em especial, geramos também uma análise PCA (Análise de Componentes Principais). Esta análise é um método multivariado que indica como sumarizar 


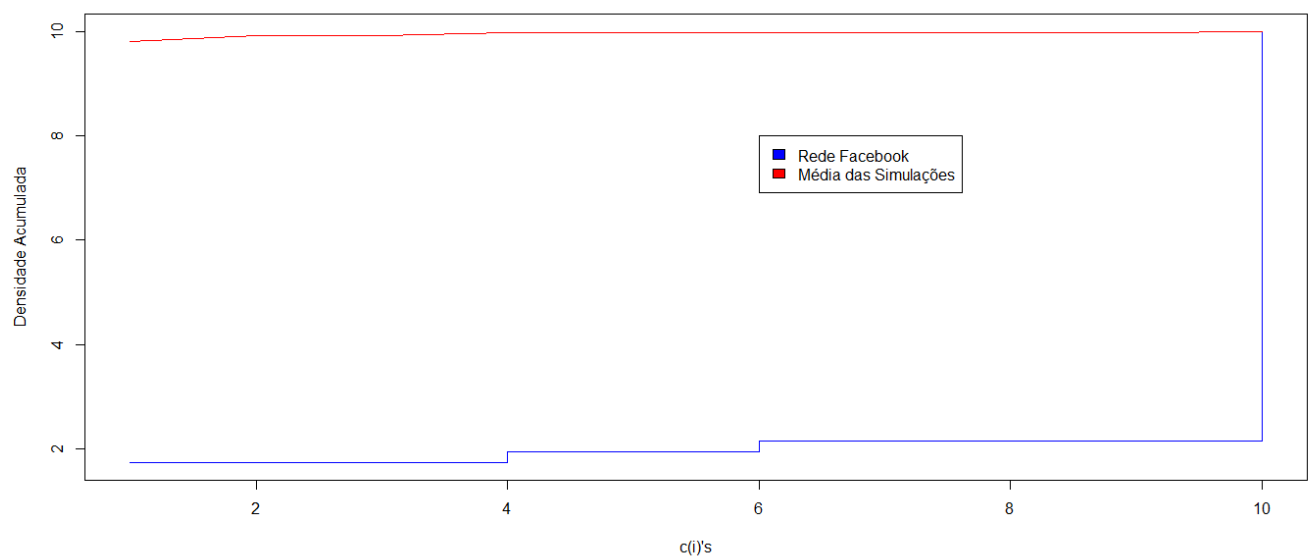

Figura 26 - Distribuição acumulada dos coeficientes de aglomeração locais Fonte: Dados da pesquisa.

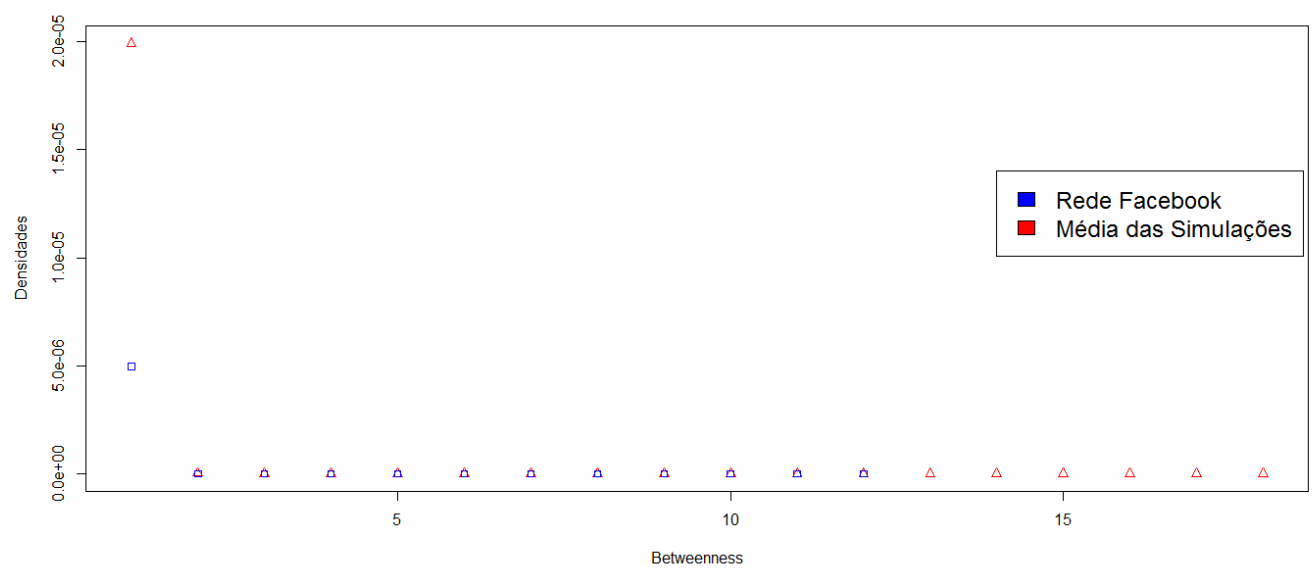

Figura 27 - Distribuições dos Betweenness

Fonte: Dados da pesquisa.

os dados que contém muitas variáveis por um conjunto menor de variáveis compostas, usando de maior variabilidade possível, derivadas a partir do conjunto original. Objetivamente falando, ela omite as informações dependentes e expõe as informações principais. Em primeiro momento realizamos o PCA apenas para as dez simulações e a rede real. O gráfico nos mostra pontos das simulações (em azul) próximos ao ponto da rede real (em vermelho), significando que a maioria das medidas nas simulações possuem valores próximos a da rede real, obtendo pouca variação.

Posteriormente, realizamos o PCA com mais dez simulações do modelo ErdösRényi (ponto em marron), do modelo Wattz-Strogatz (pontos em rosa) e do modelo Barabasi-Albert (pontos em vermelho), para comparar quais se assemelham de melhor forma com a rede real (ponto em verde). Os códigos para formar os respectivos modelos, 


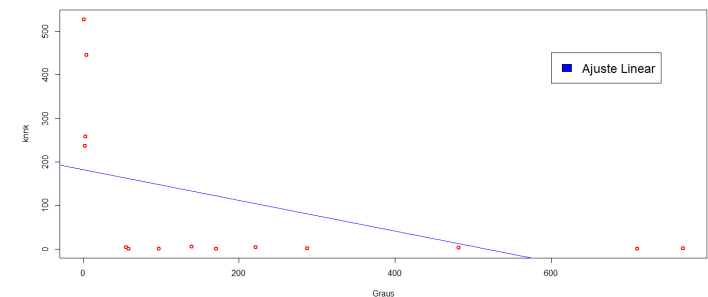

(a) Facebook

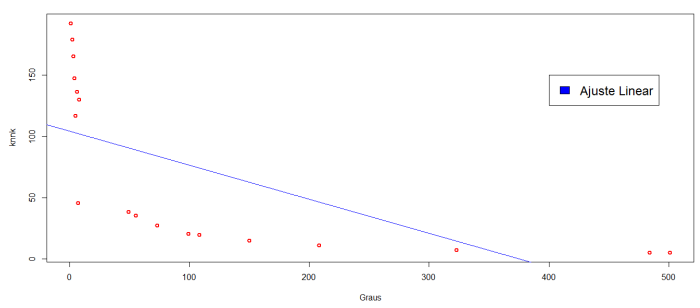

(c) $F_{2}$

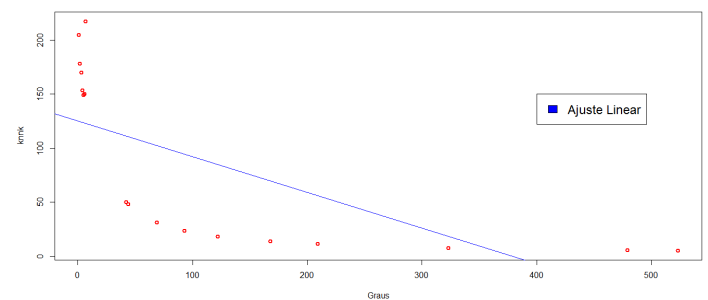

(b) $F_{1}$

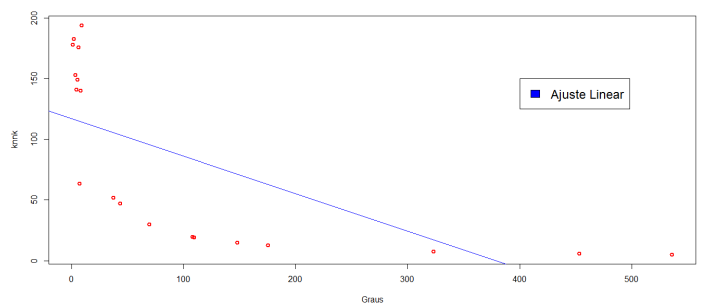

(d) $F_{3}$

Figura 28 - Graficos $k \times k_{n n}$

Fonte: Dados da pesquisa.

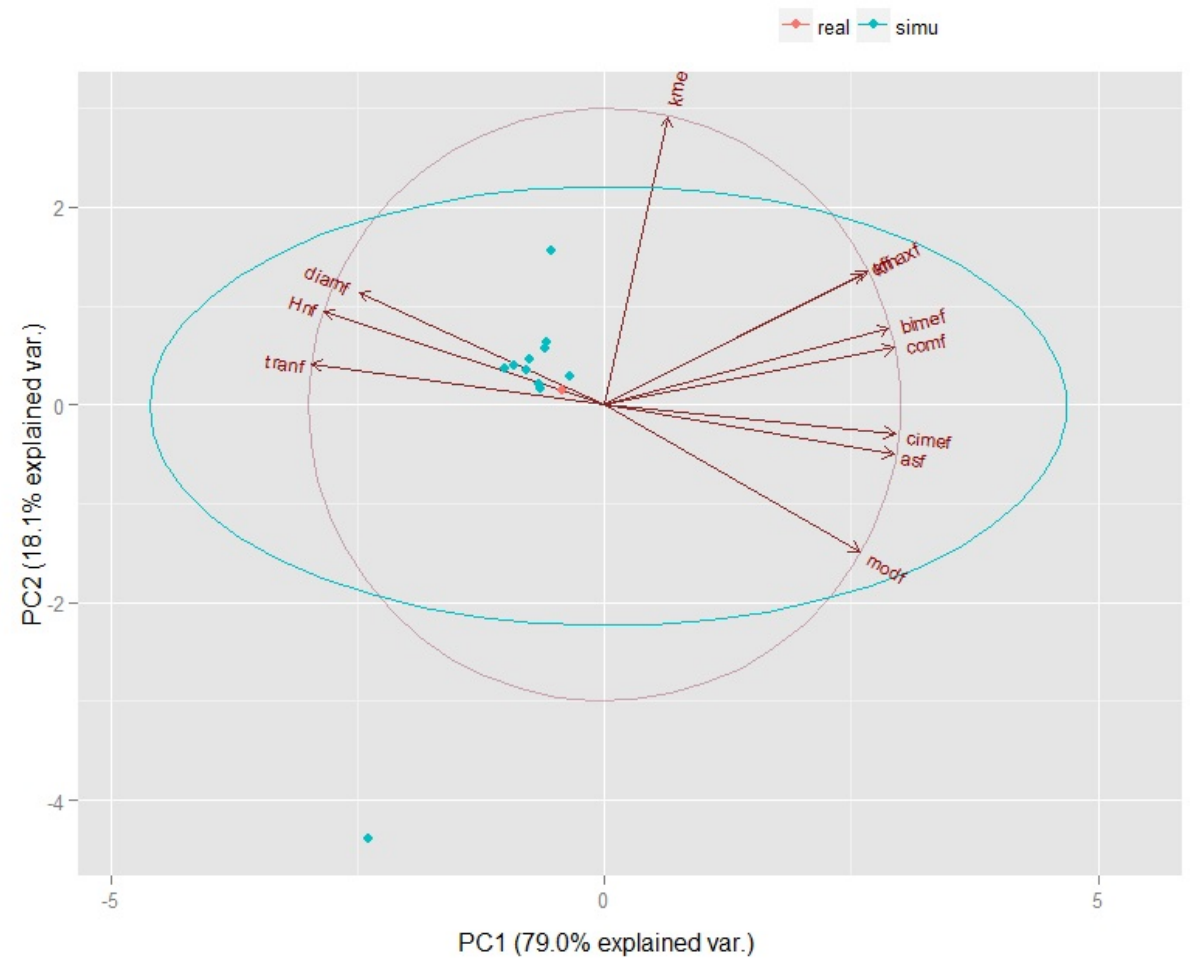

Figura $29-\mathrm{PCA}$

Fonte: Dados da pesquisa.

tendo em vista a preocupação em assemelhar o numero de vértices e arestas à rede real, podem ser vistos ao final do Anexo A. Os resultados mostram uma proximidade maior 
do modelo Kang, Pachon e Rodríguez com a rede real em relação aos outros métodos, na maioria das medidas. Trazendo um ponto positivo ao modelo no que diz respeito à simulações.

Os gráficos das análises PCA são apresentados nas Figura 29 e Figura 30.

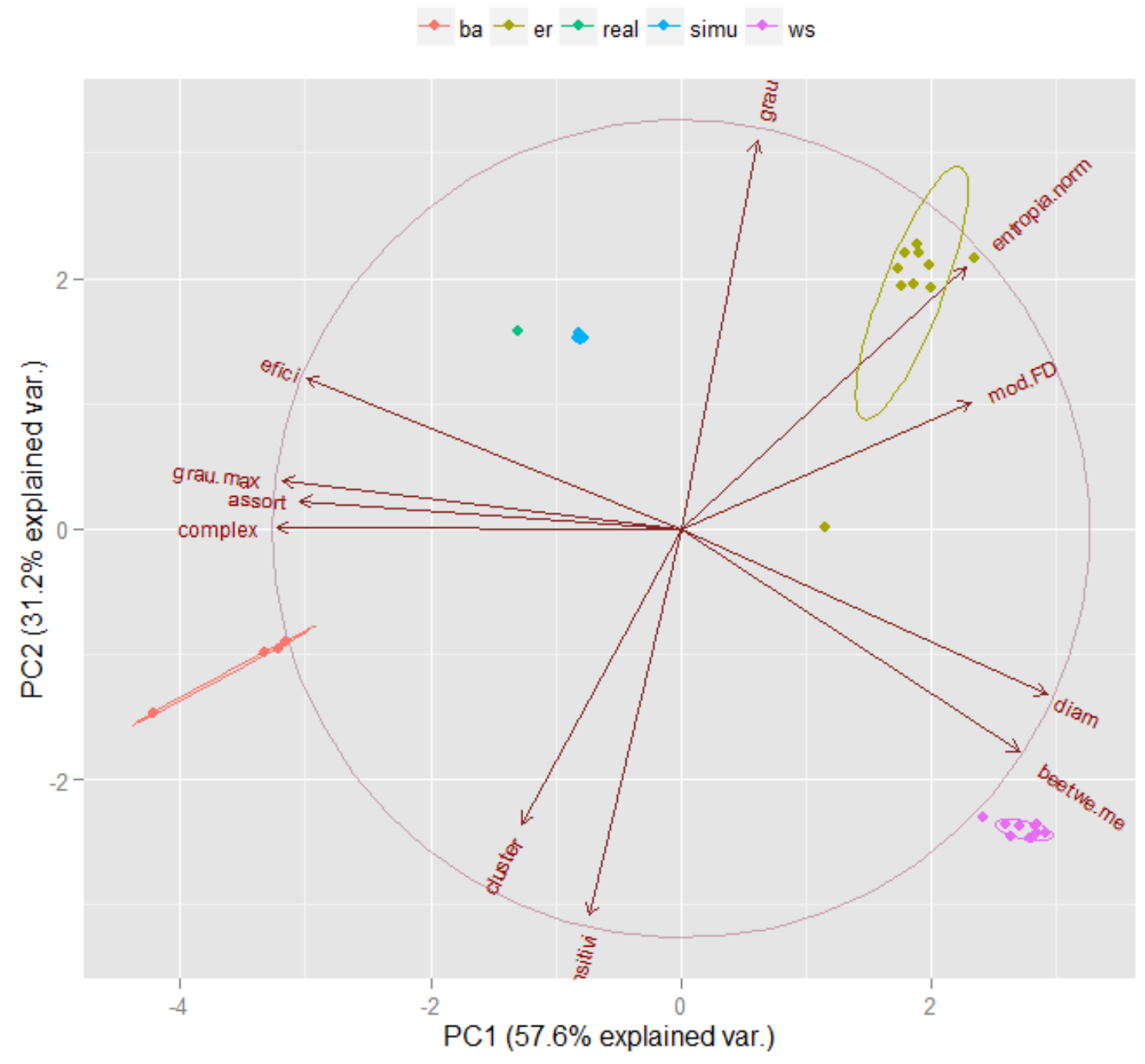

Figura 30 - PCA2

Fonte: Dados da pesquisa. 



\section{CONCLUSÃO E DISCUSSÕES}

Neste trabalho apresentamos argumentos e métodos usados para encontrar resultados assintóticos relacionados a conectividade em um modelo de grafo aleatório não homogêneo, estabelecendo pequenas restrições como a fixação do tamanho dos super-vértices por uma constante e a existência de uma proporção sobre a quantia de super-vértices de tamanho 1 em relação ao total. Para obtenção destes resultados foi necessário analisarmos a existência de pequenos componentes e o comportamento assintótico do número de supervértices isolados. Uma apresentação mais geral sobre a conectividade do modelo pode ser vista em Kang, Pachón e Rodríguez (2016), onde os autores deixam de fixar $\mu_{1}>0$ mas estabelecem novas variáveis como $i^{*}=i^{*}(N):=\left\{1 \leq i \leq r \mid k_{i}^{\frac{1}{i(n-i)}}=\max _{1 \leq j \leq r} k_{j}^{\frac{1}{j(n-j)}}\right\}$, tendo assim modificações nos limitantes de $c(N)$ e na probabilidade $p$.

Formamos três códigos diferentes com intuito de realizar simulações para o modelo, onde dois deles foram aplicados para simular duas redes reais, uma envolvendo conexões metabólicas entre proteínas e outra sobre conexões na rede facebook. Tais simulações nos proporcionaram dados para aplicarmos cálculos de medidas topológicas, aos quais conseguimos resultados extremamente satisfatórios, no que se refere á obtenção de semelhança entre a distribuição dos graus nas simulações e nas redes reais, lembrando que a escolha dos nossos parâmetros de construção do grafo foram voltadas essencialmente a obtenção desta propriedade. Outras medidas que resultaram em semelhança de distribuição foram as de centralidade. No entanto houveram demais medidas que acarretaram em resultados consideravelmente diferentes dos encontrados nas redes reais. As medidas que envolvem aglomeração e distância foram exemplos deste acontecimento, pois apresentaram níveis baixos enquanto as redes reais continham níveis mais altos. É valido lembrar que as medidas de aglomeração possuem correlação com as medidas de distância, pois quanto maior o coeficiente de aglomeração, mais triângulos estarão estabelecidos entre os vértices, sendo menor a distância entre dois vértices quaisquer selecionados. Aqui nos vêm a questão de verificar se estes níveis de aglomeração são facilmente modificados perante as escolhas dos 
parâmetros do modelo, ou se o modelo não consegue controlar tais medidas, possuindo assim um nível padrão para qualquer modo de simulação.

No geral, temos indícios de que o modelo $G(N, \mathcal{K}, p)$ possui boa eficiência em comparação aos modelos de grafos aleatórios descritos por Erdos-Renyi, Watts-Strogatz e Barabási-Albert, devido a menor variabilidade das medidas constatadas no gráfico de análise dos componentes principais. Com isso, vemos que este modelo apresenta um grande potencial de aplicação em simulação de redes reais.

Estudos posteriores podem ser feitos analisando demais propriedades do modelo ainda não estabelecidas e principalmente verificando o comportamento dos resultados de medidas topológicas de acordo com as diferentes escolhas nos parâmetros, sendo possível dar ênfase em obter melhores resultados de aglomerações, distâncias entre outros. 
BARRAT, A.; BARTHéLEMI, M.; VESPIGNANI, A. Dynamical Processes on Complex Networks. 1ํ. ed. [S.l.]: Cambridge University Press, 2008. ISBN 9780521879507. Citado na página 99.

BOCCALETTI, S.; LATORA, V.; MORENO, Y.; CHAVEZ, M.; HWANG, D.-U. Complex networks: Structure and dynamics. Physics reports, Elsevier, v. 424, n. 4, p. 175308, 2006. Citado 2 vezes nas páginas 22 e 27.

BOLLOBáS, B. Random Graphs. 2. ed. United Kingdom: Cambridge University Press, 2001. Citado na página 36.

CARvalho, M. A. G. de. Teoria dos Grafos - Uma Introdução. Limeira, SP Brasil: [s.n.], 2005. Disponível em: < http://www.ft.unicamp.br/ magic/ft024/apografos ceset_magic.pdf $>$. Acesso em: 28/10/2015. Citado na página 25.

COSTA, L. da F.; JUNIOR, O. N. O.; TRAVIESO, G.; RODRIGUES, F. A.; BOAS, P. R. V.; ANTIQUEIRA, L.; VIANA, M. P.; ROCHA, L. E. C. da. Analyzing and Modeling Real-World Phenomena with Complex Networks: A Survey of Applications. [S.1.]: Advances in Physics, 2008. Citado na página 99.

COSTA, P. P. da. Teoria de Grafos e suas Aplicações. Dissertação (Mestrado) Universidade Estadual Paulista "Júlio de Mesquita Filho", Rio Claro, SP - Brasil, 2011. Disponível em: < http://www.ft.unicamp.br/ magic/ft024/apografos_ceset_magic.pdf >. Acesso em: 28/10/2015. Citado na página 25.

CSARDI, G.; NEPUSZ, T. The igraph software package for complex network research. InterJournal, Complex Systems, p. 1695, 2006. Disponível em: <http://igraph.org $>$. Citado na página 73.

DANZIGER, P. Big O Notation. Toronto - Canada: [s.n.], 2009. Disponível em: < http:// www.scs.ryerson.ca/ mth110/Handouts/PD/bigO.pdf $>$. Acesso em: 23/11/2014. Citado na página 93.

DIEDERICHSEN, J. Taylor, Brook (1685-1731). 2015. Disponível em: < http://www. fem.unicamp.br/ em313/paginas/person/taylor.htm>. Acesso em: 25/11/2015. Citado na página 91.

DIESTEL, R. Graph Theory. Eletronic edition. New York- USA: Springer, 2000. ISBN 0-387-95014-1. Citado na página 25.

DURRETT, R. Random Graph Dynamics. 1. ed. New York: Cambridge University Press, 2007. Citado 2 vezes nas páginas 22 e 37.

ERDöS, P.; RéNYI, A. On random graphs i. Publ. Math. Debrecen, v. 6, p. 290297, Novembro 1959. Disponível em: <http://snap.stanford.edu/class/cs224w-readings/ erdos59random.pdf $>$. Citado 2 vezes nas páginas 22 e 34 . 
On the evolution of random graphs. Publ. Math. Inst. Hung. Acad. Sci, Institute of Mathematics Hungarian Academy of Sciences, Hungary, v. 5, p. 17-61, 1960. Disponível em: < http://snap.stanford.edu/class/cs224w-readings/erdos60random.pdf > . Citado na página 34.

GILBERT, E. N. Random graphs. New Jersey - USA: [s.n.], 1959. Disponível em: $<$ http://projecteuclid.org/download/pdf_1/euclid.aoms/1177706098>. Citado 2 vezes nas páginas 22 e 34 .

GONçALVES, A. L. Grafos: Aplicações ao Jogo. Dissertação (Mestrado) - Universidade Portucalense, Porto, 2007. Disponível em: <http://repositorio.uportu.pt:8080/jspui/ bitstream/11328/539/2/TMMAT\%20102.pdf $>$. Acesso em: 20/11/2014. Citado na página 25.

GUT, A. Probability: A Graduate Course. 2. ed. New York, NY: Springer, 2013. Citado na página 39.

HARARY, F. Graph Theory. Massachusetts - USA: Addison-Wesley Publishing Company, 1969. Citado 2 vezes nas páginas 21 e 25.

HARJU, T. Lecture Notes on GRAPH THEORY. Turku, Finland: [s.n.], 2011. Disponível em: <http://cs.bme.hu/fcs/graphtheory.pdf>. Acesso em: 28/10/2015. Citado na página 25.

HOFSTAD, R. van der. manuscrito, Ramdom Graphs and Complex Networks. Holanda: [s.n.], 2013. Disponível em: < http://www.win.tue.nl/ rhofstad/NotesRGCN.pdf $>$. Citado 3 vezes nas páginas 36, 37 e 44.

JAMES, B. R. Probabilidade: um curso em nível intermediário. 3. ed. Rio de Janeiro: IMPA, 2013. 304 p. ISBN 978-85-244-0101-5. Citado 2 vezes nas páginas 37 e 38 .

JANSON, S.; LUCZAK, T.; RUCINSKI, A. Random Graphs. New York: [s.n.], 2000. v. 1.43 p. Citado na página 49.

KANG, M.; PACHóN, A.; RODRíGUEZ, P. M. Evolution of a modified binomial random graph by agglomeration. arXiv preprint arXiv:1310.3148, 2016. Disponível em: <http: //arxiv.org/pdf/1310.3148.pdf>. Citado 7 vezes nas páginas 11, 13, 22, 51, 53, 69 e 85.

KUNEGIS, J. KONECT - The Koblenz Network Collection. 2013. Disponível em: $<$ http://konect.uni-koblenz.de/>. Citado na página 74 .

LEITHOLD, L. Calculo com geometria analitica. 3. ed. [S.l.]: Harbra, 1994. 684 p. ISBN 9788529400945. Citado na página 92.

LUCCHESI, C. L. Introdução à Teoria dos Grafos. Rio de Janeiro - RJ: Instituto de Matemática Pura e Aplicada, 1979. Citado na página 21.

MARSAGLIA, G.; MARSAGLIA, J. C. A new derivation of stirling's approximation to n! American Mathematical Monthly, JSTOR, p. 826-829, 1990. Citado na página 97. 
MORGENSTERN, J. Lecture Notes on "Big-O" Notation. Philadelphia - USA: [s.n.], 2012. Disponível em: <https://www.cs.cmu.edu/ jamiemmt/teaching/su-122/lectures/ 07-bigo.pdf $>$. Acesso em: 23/11/2014. Citado na página 93.

NEWMAN, M. E. The structure and function of complex networks. SIAM review, SIAM, v. 45, n. 2, p. 167-256, 2003. Citado na página 22.

PEREIRA, P. R. da C.; MARKENZON, L.; VERNET, O. Código reduzido de prüfer para k-árvores rotuladas. XXXVII Simpósio Brasileiro de Pesquisa Operacional, Gramado - RS, p. 2235-2342, 2005. Citado na página 32.

R Core Team. R: A Language and Environment for Statistical Computing. Vienna, Austria, 2013. Disponível em: <http://www.R-project.org/>. Citado na página 73.

ROSS, S. Probabilidade: um curso moderno com aplicações. 8. ed. [S.l.]: Bookman, 2010. 608 p. ISBN 978-85-7780-621-8. Citado na página 37.

SCHROEDER, S. Facebook Hits One Billion Active Users. 2012. Disponível em: <http://mashable.com/2012/10/04/facebook-one-billion/>. Citado na página 78.

SOUSA, L. O teorema das quatro cores. Instituto Politécnico de Viseu, 2001. Disponível em: <http://www.ipv.pt/millenium/Millenium24/12.pdf>. Acesso em: 22/11/2014. Citado na página 25.

SPIELMAN, D. A. A Note on Asymptotics. New Haven - CT, USA: [s.n.], 2015. Disponível em: <http://www.cs.yale.edu/homes/spielman/365/asymptotics.pdf >. Acesso em: 28/05/2015. Citado na página 93.

STEFAnES, M. A. Notação Assintótica. Mato Grosso do Sul - Brasil: [s.n.], 2007. Disponível em: <http://www.dct.ufms.br/ marco/analise2007/aula02_4.pdf>. Acesso em: 22/11/2014. Citado na página 93.

STEWART, J. Cálculo, volume II. 6. ed. São Paulo: Cengage Learning, 2011. Citado 2 vezes nas páginas 91 e 92.

SUDAKOV, B. Graph Theory. Zurich: [s.n.], 2015. Disponível em: <http: //www2.math.ethz.ch/education/bachelor/lectures/fs2015/math/graph_theory/graph_ theory_notes.pdf $>$. Acesso em: 28/10/2015. Citado na página 25. 

APÊNDICE

A

SÉRIES DE TAYLOR E MACLAURIN

As séries de Taylor foram desenvolvidas pelo matemático inglês Brook Taylor (16851731), que se tornou membro da Royal Society de Londres em 1712. Entre seus interesses estavam os estudos de oscilações de corpos, capilaridade, magnetismo, termodinâmica e é claro, expansão de funções em séries infinitas (DIEDERICHSEN, 2015).

As demais informações aqui apresentadas foram baseadas em Stewart (2011). Uma série de Taylor é uma série de funções da seguinte forma:

$$
f(x)=\sum_{n=0}^{\infty} a_{n}(x-a)^{n}
$$

onde

$$
a_{n}=\frac{f^{(n)}(a)}{n !} .
$$

a constante $a$ é chamada de centro da série, ela pode ser encarada como uma função real ou complexa. Caso $a=0$ a série é também chamada de série de Maclaurin.

A série de Taylor associada a uma função $f$ infinitamente diferenciável (real ou complexa) definida em um intervalo aberto $(a-r, a+r)$ é a série de potências dada por

$$
f(x)=\sum_{n=0}^{\infty} \frac{f^{(n)}(a)}{n !}(x-a)^{n},
$$

onde $f^{(n)}(a)$ denota a n-ésima derivada de $f$ no ponto $a$.

Utilizando desta ferramenta, podemos moldar várias funções, como por exemplo:

- $e^{x}=\sum_{n=0}^{\infty} \frac{x^{n}}{n !}, \quad \forall x$

- $\ln (1+x)=\sum_{n=0}^{\infty} \frac{(-1)^{n}}{n+1} x^{n+1}, \quad$ para $|x|<1$;

- $\ln (1-x)=\sum_{n=0}^{\infty}-\frac{x^{n+1}}{n+1} \quad$ para $|x|<1$; 
- $\frac{x^{m}}{1-x}=\sum_{n=m}^{\infty} x^{n}, \quad$ para $|x|<1$;

- $(1+x)^{\alpha}=\sum_{n=0}^{\alpha}\left(\begin{array}{l}\alpha \\ n\end{array}\right) x^{n}, \quad$ para todo $|x|<1$ e todo complexo $\alpha$;

- $\cos (x)=\sum_{n=0}^{\infty} \frac{(-1)^{n}}{(2 n) !} x^{2 n} \quad \forall x$

- $\sin (x)=\sum_{n=0}^{\infty} \frac{(-1)^{n}}{(2 n+1) !} x^{2 n+1} \quad \forall x$.

Para mais informações ver Stewart (2011, p. 698-708) ou Leithold (1994). 
APÊNDICE

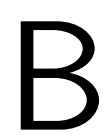

\section{NOTAÇÃO ASSINTÓTICA E FÓRMULA DE STIRLING}

\section{Símbolos de Landau}

Os símbolos de Landau são notações utilizadas para auxiliar nas teorias matemáticas que descrevem o comportamento assintótico de funções, logo também são conhecidos como notação assintótica. Eles são geralmente utilizados para simplificar erros de aproximação, facilitando os cálculos com operações. Estes símbolos foram desenvolvido nos anos de 1990 pelos matemático germânicos Paul Bachmann (1837-1920) e Edmund Landau (1877-1938) para abreviar a noção de que uma função era "da mesma ordem de" ou "de ordem inferior a" outra função na vizinhança de um ponto. Este apêndice foi baseado em Spielman (2015), Morgenstern (2012), Stefanes (2007) e Danziger (2009).

Definição B.0.1. Considere duas funções reais $f$ e $g$.

- A função $f(x)$ é dita grande $\mathrm{O}$ de $g(x)$, notação $(f(x)=O(g(x)))$, se existem duas constantes positivas $c$ e $x_{0}$ tais que

$$
|f(x)| \leq c|g(x)| \quad \forall x>x_{0} .
$$

- A função $f(x)$ é dita grande Omega de $g(x),(f(x)=\Omega(g(x)))$, se existem duas constantes positivas $c$ e $x_{0}$ tais que

$$
|f(x)| \geq c|g(x)| \quad \forall x>x_{0} .
$$

- A função $f(x)$ é dita grande Theta de $g(x),(f(x)=\Theta(g(x)))$, se existem constantes positivas $c_{1}, c_{2}$ e $x_{0}$ tais que

$$
c_{2}|g(x)| \leq|f(x)| \leq c_{1}|g(x)| \quad \forall x>x_{0}
$$


- A função $f(x)$ é dita pequeno o de $g(x),(f(x)=o(g(x)))$, se existe uma constante positiva $x_{0}$ tal que

$$
|f(x)|<c|g(x)| \quad \forall c>0, \quad x>x_{0}
$$

Note que, caso uma função $f$ seja $o(1)$, teremos

$$
-c<f(x)<c \quad \forall c>0, x>x_{0},
$$

o que nos $\operatorname{diz}$ que $\lim f(x)=0$ pois podemos tomar constantes $c$ muito pequenas.

Dentre os símbolos, um dos mais importantes é a relação grande $\mathrm{O}$, denotada assim pois a taxa de crescimento de uma função também é chamada de ordem. Esta relação possui as seguintes propriedades: Sejam $f, g, h$ e $k$ funções reais.

1. $O(f) O(g)=O(f g)$;

2. $O(f)+O(g)=O(|f|+|g|)$;

3. $O(f) g=O(f g)$;

4. $O(f)+g=O(|f|+|g|)$;

5. $c O(f)=O(c f)=O(f)$, para $c \in \mathbb{R}, c \neq 0$;

6. Seja $f(x)=x$, então $x O(f)=O\left(x^{2}\right)$;

7. $O(O(f))=O(f)$;

8. Se $f=O(g)$ e $g=O(h)$ então $f=O(h)$;

9. Se $f=O(h)$ e $g=O(k)$ então $f(x)+g(x)=O(G(x))$, onde $G(x)=\max \{|h(x)|,|k(x)|\}$;

10. SE $f=O(h)$ e $g=O(h)$ então $f(x)+g(x)=O(h)$;

11. Se $f(x) \rightarrow 0$ para algum $x \rightarrow a$, então $O(f(x)) \rightarrow 0$ para $x \rightarrow a$.

Demonstração. Segue as provas de cada item respectivamente:

1. Pela definição temos $O(f) \leq c_{1}|f|$ e $O(g) \leq c_{2}|g|$ então

$$
O(f) O(g) \leq c_{1} c_{2}|f||g|=c|f g| ;
$$

2. Se $O(f) \leq c_{1}|f|$ e $O(g) \leq c_{2}|g|$, então

$$
O(f)+O(g) \leq c_{1}|f|+c_{2}|g|,
$$

tome $c=\max \left\{c_{1}, c_{2}\right\}$, logo

$$
O(f)+O(g) \leq c|f|+c|g|=c(|f|+|g|) ;
$$


3. Se $O(f) \leq c|f|$, então

$$
O(f) g \leq c|f| g \leq c|f g|
$$

4. Se $O(f) \leq c_{1}|f|$, então

$$
O(f)+g \leq c_{1}|f|+g
$$

tome $c=\max \left\{c_{1}, 1\right\} \log \mathrm{O}$

$$
O(f)+g \leq c|f|+c g \leq c|f|+c|g|=c(|f|+|g|)
$$

5. $c O(f) \leq c c_{1}|f| \leq c_{1}|c f|=k|f|$, onde $k$ é constante;

6. $O(f) \leq c|x|$, então

$$
x O(f) \leq x c|x|=c\left|x^{2}\right|
$$

7. $O(O(f)) \leq c_{1}|O(f)| \leq c_{1} c_{2}|f|=k|f|$, onde $k=c_{1} c_{2}$ constante;

8. Seja $f=O(g)$ e $g=O(h)$, então

$$
f=O(g) \leq c_{1}|O(h)| \leq c_{1} c_{2}|h|,
$$

$\log 0 f=O(h)$

9. Seja $f=O(h(x))$ e $g=O(k(x))$, então

$$
f+g \leq c_{1}|h(x)|+c_{2}|k(x)| \leq c_{1} \max \{|h(x)|,|k(x)|\}+c_{2} \max \{|h(x)|,|k(x)|\},
$$

assim,

$$
f+g \leq\left(c_{1}+c_{2}\right) \max \{|h(x)|,|k(x)|\}
$$

10. Seja $f=O(h)$ e $g=O(h)$, então

$$
f+g \leq c_{1}|h|+c_{2}|h|=\left(c_{1}+c_{2}\right)|h|
$$

11. Seja $g=O(f(x))$, então $|g| \leq c|f(x)|$, ou seja,

$$
-c|f(x)| \leq g \leq c|f(x)|,
$$

aplicando um limite onde $f(x) \rightarrow 0$ temos,

$$
\lim _{x \rightarrow a}-c|f(x)| \leq \lim _{x \rightarrow a} g \leq \lim _{x \rightarrow a} c|f(x)|,
$$

o que nos da $0 \leq \lim _{x \rightarrow a} g \leq 0$, ou seja,

$$
\lim _{x \rightarrow a} g=\lim _{x \rightarrow a} O(f(x))=0 .
$$


Apresentamos a seguir alguns exemplos que utilizam as propriedades de notação assintótica juntamente com as séries de Taylor.

Exemplo B.0.1. Vamos verificar que $1-x=e^{-x+O\left(x^{2}\right)}$ para $|x|<1$. Para isso, utilizamos da expansão de Taylor de $\ln (1-x)$,

$$
\begin{aligned}
\ln (1-x) & =\sum_{n=0}^{\infty}-\frac{x^{n+1}}{n+1} \quad \text { para }|x|<1 \\
& =-x-\frac{x^{2}}{2}-\frac{x^{3}}{3}-\frac{x^{4}}{4}-\frac{x^{5}}{5}-\ldots \\
& =-x-O\left(x^{2}\right) \\
& =-x+O\left(x^{2}\right) .
\end{aligned}
$$

Exemplo B.0.2. Vamos provar que $e^{O\left(x^{2}\right)}=1+O\left(x^{2}\right)$, utilizando da expansão de Taylor da função exponencial.

$$
\begin{aligned}
e^{O\left(x^{2}\right)} & =1+O\left(x^{2}\right)+\frac{\left(O\left(x^{2}\right)\right)^{2}}{2}+\frac{\left(O\left(x^{2}\right)\right)^{3}}{3 !}+\frac{\left(O\left(x^{2}\right)\right)^{4}}{4 !}+\ldots \\
& =1+O\left(O\left(x^{2}\right)\right) \\
& =1+O\left(x^{2}\right) .
\end{aligned}
$$

\section{Fórmula de Stirling}

A fórmula de Stirling foi desenvolvida pelo matemático escocês James Stirling (1692-1770) e é uma poderosa aproximação para a função fatorial, onde é expressado que

$$
n ! \approx n^{n+1 / 2} e^{-n} \sqrt{2 \pi}
$$

Existem duas formas mais conhecidas para provar esta aproximação. Nós iremos realizar a prova que se utiliza da função Gamma, na qual, para $t \in \mathbb{C} \operatorname{com} \operatorname{Re}(t)>0$

$$
\Gamma(t)=\int_{0}^{\infty} x^{t-1} e^{-x} d x
$$

Usando integral por partes, a função Gamma apresenta a propriedade

$$
\Gamma(t+1)=t \Gamma(t)
$$

e combinando isto com $\Gamma(1)=1$, temos

$$
\Gamma(n)=1.2 \ldots(n-1)=(n-1) !,
$$

para todo $n \in \mathbb{N}$.

Com isso, usando a função Gamma e integração por partes obtemos

$$
n !=\int_{0}^{\infty} x^{n} e^{-x} d x
$$


Reescrevendo a equação e tomando $x=n y$ temos

$$
n !=\int_{0}^{\infty} e^{n \ln x-x} d x=e^{n \ln n} n \int_{0}^{\infty} e^{n(\ln y-y)} d y .
$$

Aplicando o método de Laplace obtemos

$$
\int_{0}^{\infty} e^{n(\ln y-y)} d y \approx \sqrt{\frac{2 \pi}{n}} e^{-n} .
$$

Logo a aproximação para $n$ ! resulta em

$$
\begin{aligned}
n ! & \approx e^{n \ln n} n \sqrt{\frac{2 \pi}{n}} e^{-n} \\
& =n^{n} e^{-n} \sqrt{2 \pi} \frac{n}{\sqrt{n}} \\
& =n^{n+1 / 2} e^{-n} \sqrt{2 \pi} .
\end{aligned}
$$

Para mais informações ver Marsaglia e Marsaglia (1990). 

APÊNDICE
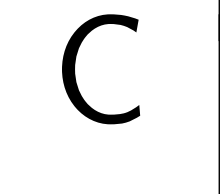

\section{MEDIDAS TOPOLÓGICAS}

Vamos apresentar alguns conceitos, medidas e métodos que fornecem informações relevantes para a classificação dos diferentes tipos de redes. Tais informações podem ser vistas em Costa et al. (2008) e Barrat, Barthélemi e Vespignani (2008).

\section{Conectividade}

Na Seção 2.1 já definimos o grau de um vértice. Sendo assim, podemos verificar sua distribuição $\mathbb{P}\left(D_{v}=k\right)$, definida como sendo a probabilidade de que um vértice escolhido de maneira uniforme possua um determinado grau $k$. A descrição e representação gráfica desta distribuição pode ser feita por meio da construção de um histograma. Um histograma é um gráfico de colunas onde a área de cada coluna representa a porcentagem do elemento analisado em relação ao todo, um exemplo é feito na Figura 31.

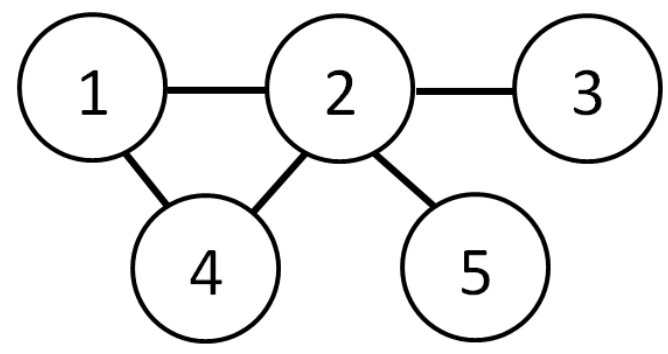

G

(a) Grafo G

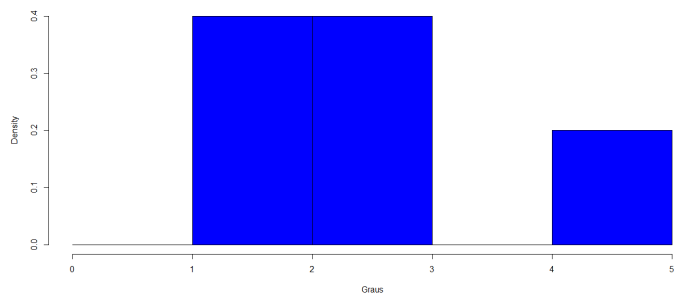

(b) Histograma dos graus de $\mathrm{G}$

Figura 31 - Modelo de Histograma

Fonte: Elaborada pelo autor. 
Outra medida que podemos utilizar é o grau médio, expresso por

$$
\mathbb{E}\left[D_{v}\right]=\sum_{k=0}^{n-1} k \mathbb{P}\left(D_{v}=k\right)
$$

Definição C.0.1. Dada a distribuição dos graus de um grafo $G$, é possível definir os m-ésimos momentos em relação aos graus. Seja $|V(G)|=n$, temos que

$$
\mathbb{E}\left[D_{v}^{m}\right]=\sum_{k=0}^{n-1} k^{m} \mathbb{P}\left(D_{v}=k\right)=\frac{1}{n} \sum_{i=1}^{n}\left(d\left(v_{i}\right)\right)^{m} .
$$

Com isso, o segundo momento do grau é dado por:

$$
\mathbb{E}\left[D_{v}^{2}\right]=\sum_{k=0}^{n-1} k^{2} \mathbb{P}\left(D_{v}=k\right)=\frac{1}{n} \sum_{i=1}^{n}\left(d\left(v_{i}\right)\right)^{2}
$$

Definição C.0.2. A entropia de Shannon é uma medida global que também está relacionada a distribuição dos graus de um grafo e é expressa por

$$
H=H(G)=-\sum_{k=0}^{n-1} \mathbb{P}\left(D_{v}=k\right) \ln \left(\mathbb{P}\left(D_{v}=k\right)\right),
$$

onde por convenção, assumimos que $0 \cdot \ln (0)=0$.

Analisando a expressão da entropia temos que seu valor mínimo é igual a 0 quando todos os vértices possuírem o mesmo valor de grau $k$, e seu valor máximo sera igual a $\ln (n)$, onde $|V(G)|=n$, quando todos os vértices tiverem valores de grau diferentes.

Para uma melhor interpretação podemos normalizar esta medida, utilizando do conhecimento sobre seu valor máximo, assim obtemos a entropia Normalizada dada por

$$
\tilde{H}=\tilde{H}(G)=-\frac{1}{\ln (n)} \sum_{k=0}^{n-1} \mathbb{P}\left(D_{v}=k\right) \ln \left(\mathbb{P}\left(D_{v}=k\right)\right)
$$

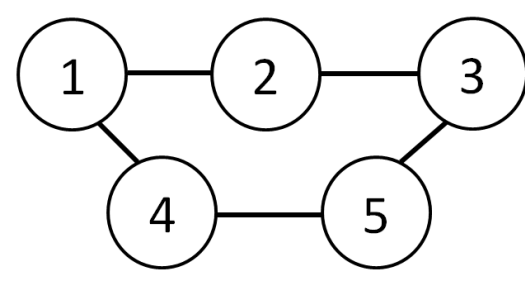

Entropia normalizada $=0$

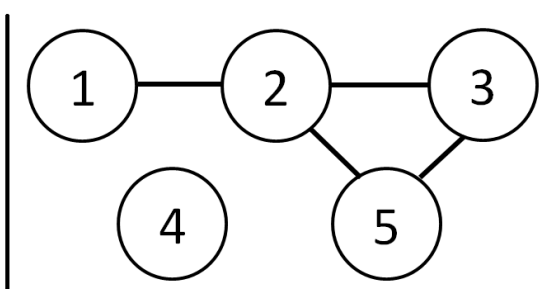

Entropia normalizada $=0,6554588$

Figura 32 - Exemplos de cálculos da entropia normalizada Fonte: Elaborada pelo autor. 


\section{Aglomeração e transitividade}

Estes conceitos utilizam de medições envolvendo a quantia de ciclos de ordem 3 existentes e um grafo (triângulos). A ideia aqui é analisar a conectividade em relação aos vizinhos de cada vértice. Por exemplo, se um vértice $v$ possui apenas dois vizinhos, e estes dois vizinhos estão conectados entre si, então o nível de aglomeração é alto. Caso os vizinhos de $v$ não sejam conectados a aglomeração diminui. Podemos interpretar da seguinte forma, digamos que eu queira realizar uma festa apenas entre pessoas que são amigas entre si, almejando uma melhor confraternização. Caso os meus amigos sejam amigos entre si, então eu posso fazer uma festa convidando todos, gerando uma maior aglomeração. Entretanto se dois de meus amigos não são amigos entre eles, não poderei convidá-los, obtendo uma menor aglomeração de pessoas. A Figura 33 mostra grafos com níveis de aglomerações diferentes, visando melhor entendimento.
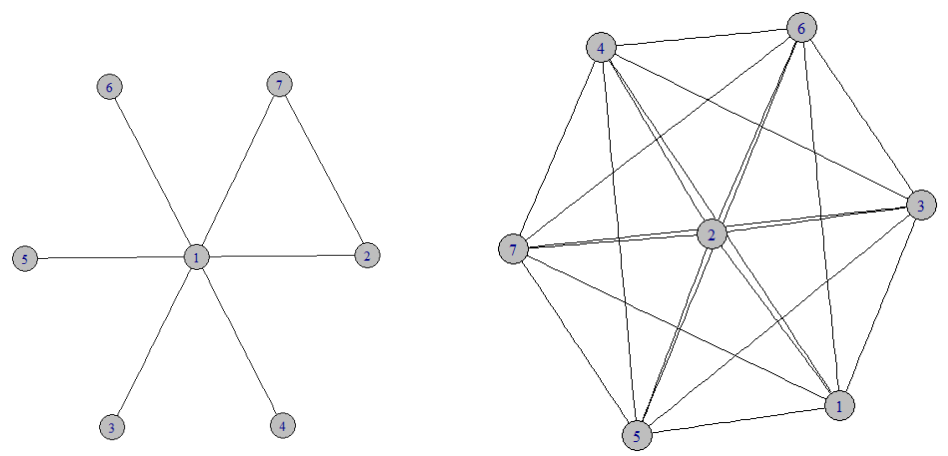

(a) Grafo com aglomeração (b) Grafo com aglomeração alta baixa

Figura 33 - Exemplos para aglomerações

Fonte: Elaborada pelo autor.

Definição C.0.3. O coeficiente de aglomeração global ou transitividade é a medida que indica a fração de ciclos de ordem 3 em um grafo, ou seja, a fração de triângulos com relação a conexão entre 3 vértices por meio de um caminho. Sua expressão é dada por

$$
C_{\Delta}=\frac{3 N_{\Delta}}{N_{3}}
$$

onde $N_{\Delta}$ é o n⿳⺈ de triângulos do grafo e $N_{3}$ é o $\mathrm{n}^{\mathrm{O}}$ de triplas conectadas (caminho com 3 vértices).

A Figura 34 apresenta dois grafos com mesmo número de vértices mas com transitividade diferentes.

Definição C.0.4. Chamamos de Clustering coefficient (coeficiente de aglomeração local) a medida local que mede a quantia de triângulos existentes conectados a um vértice 

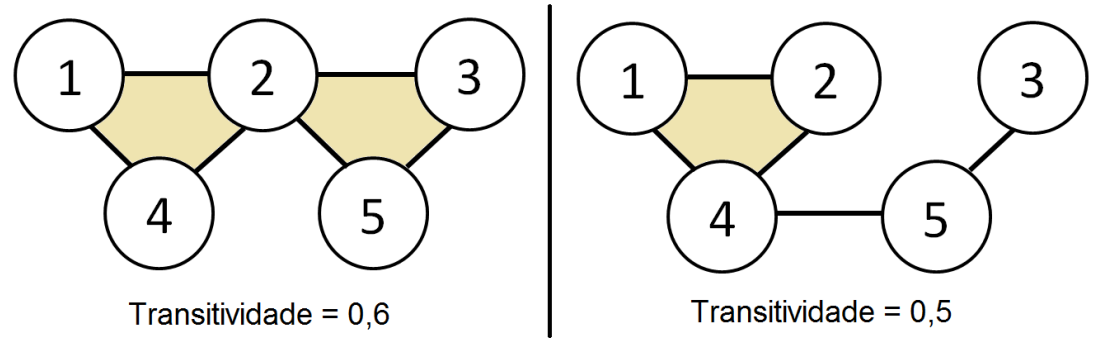

Figura 34 - Transitividades

Fonte: Elaborada pelo autor.

em relação ao máximo de triângulos possíveis de serem criados. Sua expressão é dada por

$$
c(i)=\frac{e_{i}}{d\left(v_{i}\right)\left(d\left(v_{i}\right)-1\right) / 2}
$$

onde $e_{i}$ representa o número de conexões entre os vizinhos do vértice $v_{i}$.

Note que o denominador da expressão anterior é a quantia de triângulos existentes caso todos os vizinhos de $v_{i}$ sejam conectados entre si, ou seja, é a quantia de triângulos caso o subgrafo formado por $v_{i}$ e seus vizinhos fosse um grafo completo. Note também que, se tivermos todo o grafo $G$ completo, todos os coeficientes de aglomeração locais de cada vértice serão iguais a 1 , veja Figura 35.
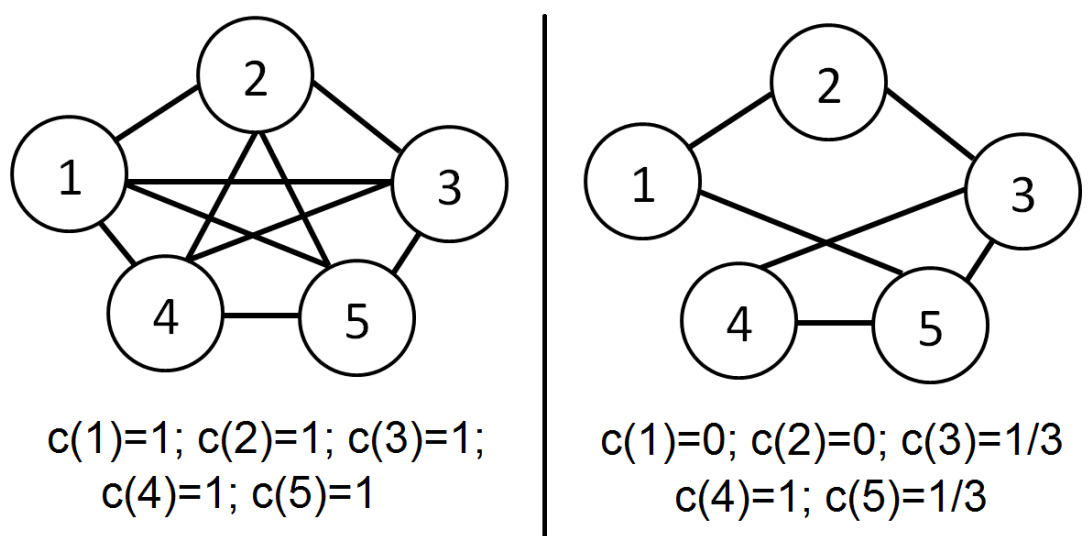

Figura 35 - Coeficientes de correlação locais

Fonte: Elaborada pelo autor.

Outra medida interessante para uma caracterização global é, naturalmente, estipular a média dos coeficientes de aglomeração locais. Denotamos assim a variável aleatória $C$ como sendo o coeficiente de correlação local de um vértice tomado aleatoriamente. Com isso, calculamos

$$
\mathbb{E}[C]=\frac{1}{n} \sum_{i=1}^{n} c(i)
$$

para um grafo $G$ onde $n=|V(G)|$. 


\section{Distâncias}

Anteriormente, no Capítulo 2, definimos caminhos por uma sequência de vértices conectados. Definimos também que o comprimento de um caminho é igual ao número de arestas visitadas no percurso.

Definição C.0.5. Um caminho geodésico ou menor caminho é definido como o caminho de menor comprimento entre dois vértices. Vamos denotar por $l_{i j}$ o caminho geodésico entre os vértices $v_{i}$ e $v_{j}$.

Definição C.0.6. Podemos expressar os caminhos geodésicos dos vértices por uma matriz de distâncias $\widehat{L}$, onde seus elementos são os valores $l_{i j}$. Com isso, podemos calcular a distribuição $\mathbb{P}\left(L_{i j}=k\right)$, que indica a probabilidade de um caminho geodésico entre dois vértices tomados ao acaso possuir valor igual a $k$. Também podemos usar da média de caminhos geodésicos para uma medida global. Seu valor é calculado como

$$
\mathbb{E}\left[L_{i j}\right]=\frac{1}{n(n-1)} \sum_{i, j} l_{i j} .
$$

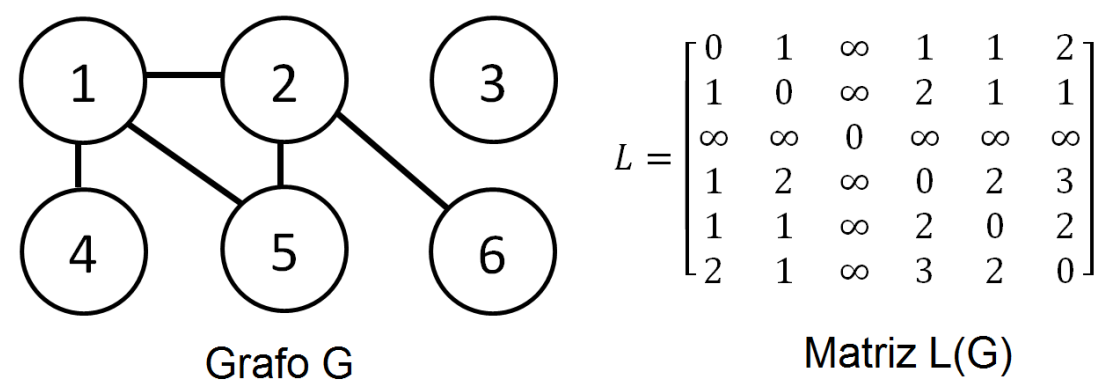

Figura 36 - Caminhos Geodésicos

Fonte: Elaborada pelo autor.

Algumas medidas podem ser definidas em relação aos menores caminhos. O diâmetro, por exemplo.

Definição C.0.7. O diâmetro de um grafo é o maior caminho geodésico que ele possui. Isto é,

$$
\operatorname{Diam}=\operatorname{Diam}(G)=\max _{i, j} l_{i j}
$$

Caso o grafo possua um vértice isolado $v_{i}$, consideramos que todos os caminhos entre $v_{i}$ e os demais vértices resultam em $\infty$. Isso pode causar problemas ao calcular a média dos menores caminhos. Para resolver isto é que estabelecemos a seguinte medida.

Definição C.0.8. Denominamos eficiência a medida global expressa por

$$
E=E(G)=\frac{1}{n(n-1)} \sum_{i \neq j} \frac{1}{l_{i j}} .
$$


Note que quanto mais alto o valor da eficiência, mais fácil será de transportar uma informação de um local para outro na rede. Logo, a interpretação destas medidas ajudam a compreender como se dá o tráfego de informação de uma determinada rede.

\section{Centralidade}

As medidas de centralidade estão relacionadas com o conceito de estipular valores de importância para cada vértice da rede, dependendo do que a pessoa que esta trabalhando com a rede estipule como sendo importante.

Um destes tipos de importância pode ser considerando a capacidade de propagar uma determinada informação mais rapidamente ao longo da rede do que os demais vértices, assim podemos considerar que um vértice com grau maior é mais importante do que um vértice com grau menor. Logo, o grau se torna uma medida de centralidade.

Outra forma pode ser relacionada com a carga que um vértice recebe, ou seja, se ele está ou não entre muitos menores caminhos que ligam os demais vértices. Para isso definimos a seguinte medida

Definição C.0.9. Definimos o grau de intermediação ou betweenness centrality como uma medida local expressa por

$$
b(i)=\sum_{\substack{j, k \\ j \neq k \neq i}} \frac{\tau_{j k}(i)}{g_{j k}}
$$

onde $\tau_{j k}(i)$ é o número de menores caminhos de $v_{j}$ até $v_{k}$ que passam por $v_{i} \operatorname{com} i \neq j \neq k$, e $g_{j k}$ é o número total de menores caminhos de $v_{j}$ até $v_{k}$.

Um exemplo dos cálculos para os betweenness pode ser visto na Figura 37

A média dos betweenness pode ser considerada uma medida global, tendo assim

$$
\mathbb{E}[B]=\frac{1}{n} \sum_{i=1}^{n} b(i)
$$

onde $n=|V(G)|$.

\section{Assortatividade}

Em redes reais temos a tendência de que vértices altamente conectados possam estar ligados. Se vértices com graus parecidos se conectam, dizemos que a rede é assortativa positiva. Por outro lado se tivermos hubs que se conectam com vértices de grau baixo, dizemos que a rede é assortativa negativa. Caso não haja relação entre o grau dos vértices com o grau de seus vizinhos, dizemos que a rede é dissortativa. 


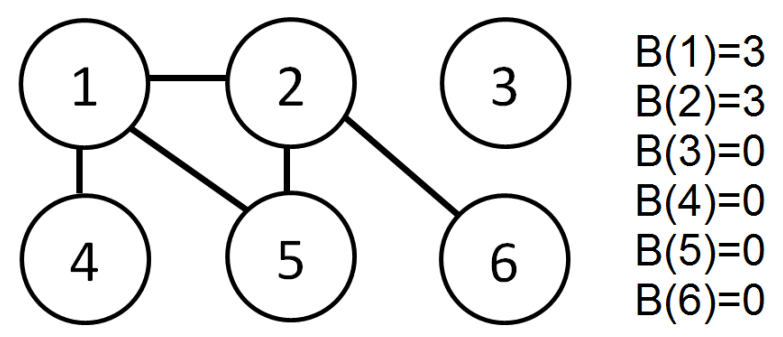

Figura 37 - Betweenness

Fonte: Elaborada pelo autor.

Uma maneira de analisar a correlação entre os graus do vértice com os graus de seus vizinhos é utilizar do coeficiente de correlação de Pearson, dado por:

$$
\rho=\frac{\operatorname{cov}(X, Y)}{\sigma_{X} \sigma_{Y}}
$$

para $X, Y$ sendo variáveis aleatórias.

Em redes, utilizamos outra expressão derivada do coeficiente de Pearson.

Definição C.0.10. O coeficiente de assortatividade é uma medida global expressa por

$$
A S=A S(G)=\frac{\frac{1}{|E(G)|} \sum_{j>i} d\left(v_{i}\right) d\left(v_{j}\right) a_{i j}-\left[\frac{1}{|E(G)|} \sum_{j>i} \frac{1}{2}\left(d\left(v_{i}\right)+d\left(v_{j}\right)\right) a_{i j}\right]^{2}}{\frac{1}{|E(G)|} \sum_{j>i} \frac{1}{2}\left(d\left(v_{i}\right)^{2}+d\left(v_{j}\right)^{2}\right) a_{i j}-\left[\frac{1}{|E(G)|} \sum_{j>i} \frac{1}{2}\left(d\left(v_{i}\right)+d\left(v_{j}\right)\right) a_{i j}\right]^{2}},
$$

onde $a_{i j}$ são os valores da matriz de adjacência $A$, definida no Capítulo 2.

Uma boa análise de assortatividade também é feita observando o gráfico $k \times k_{n n}$ que relaciona os valores dos graus no eixo $x$ com a média aritmética da esperança dos graus dos vizinhos no eixo $y$. Este gráfico pode ser obtido usando o software R, juntamente com o pacote igraph, utilizando o comando graph.knn $(\mathbf{G}) \$ \mathbf{k n n k}$, onde $G$ é o grafo a ser analisado. Exemplos de gráficos $k \times k_{n n}$ são dados na Figura 38 . 


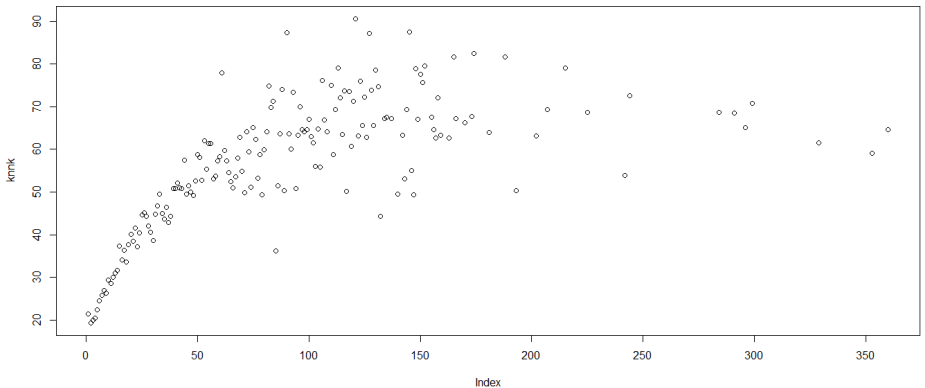

(a) Rede assortativa positiva

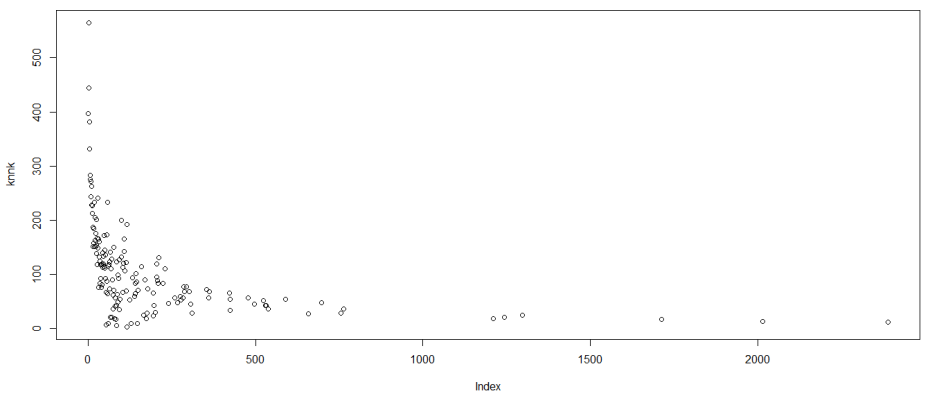

(b) Rede assortativa negativa

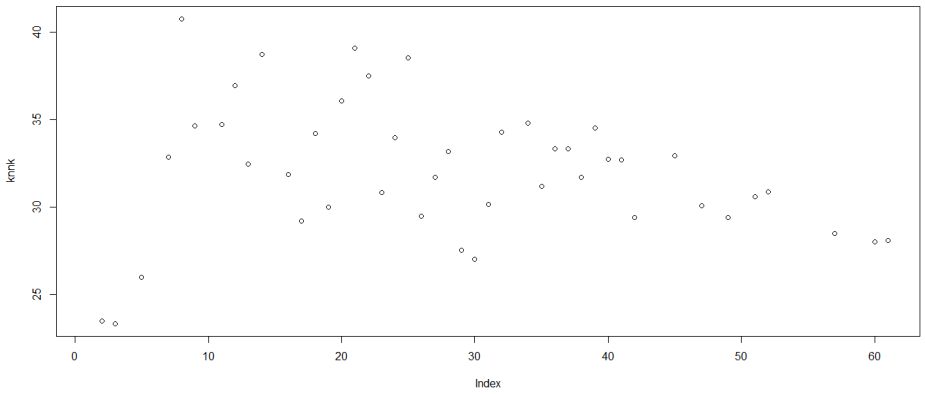

(c) Rede dissortativa

Figura 38 - Exemplos de gráficos $k \times k_{n n}$

Fonte: Elaborada pelo autor. 


\section{CÓDIGOS PARA SIMULAÇÕES NO SOFTWARE R}

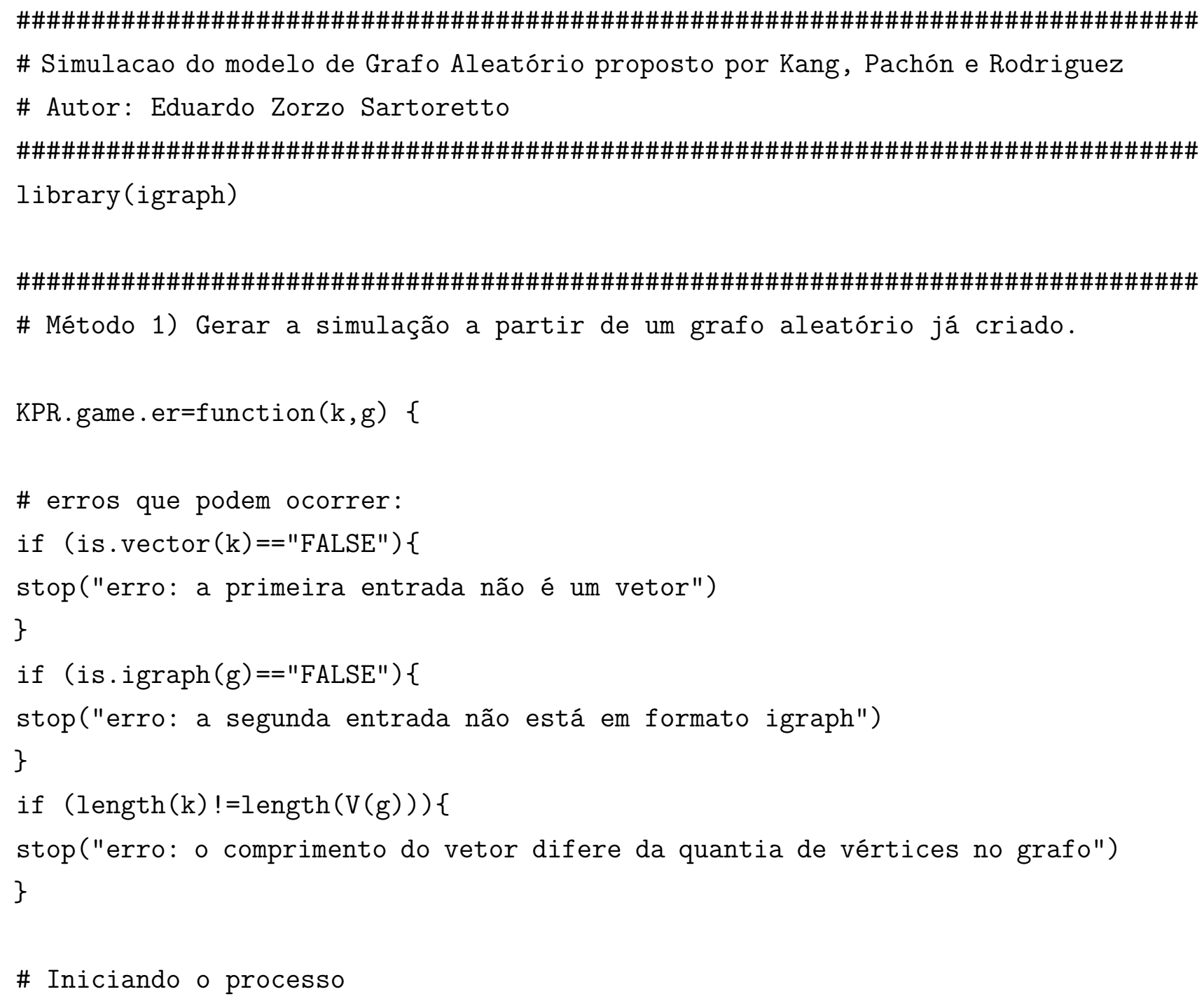




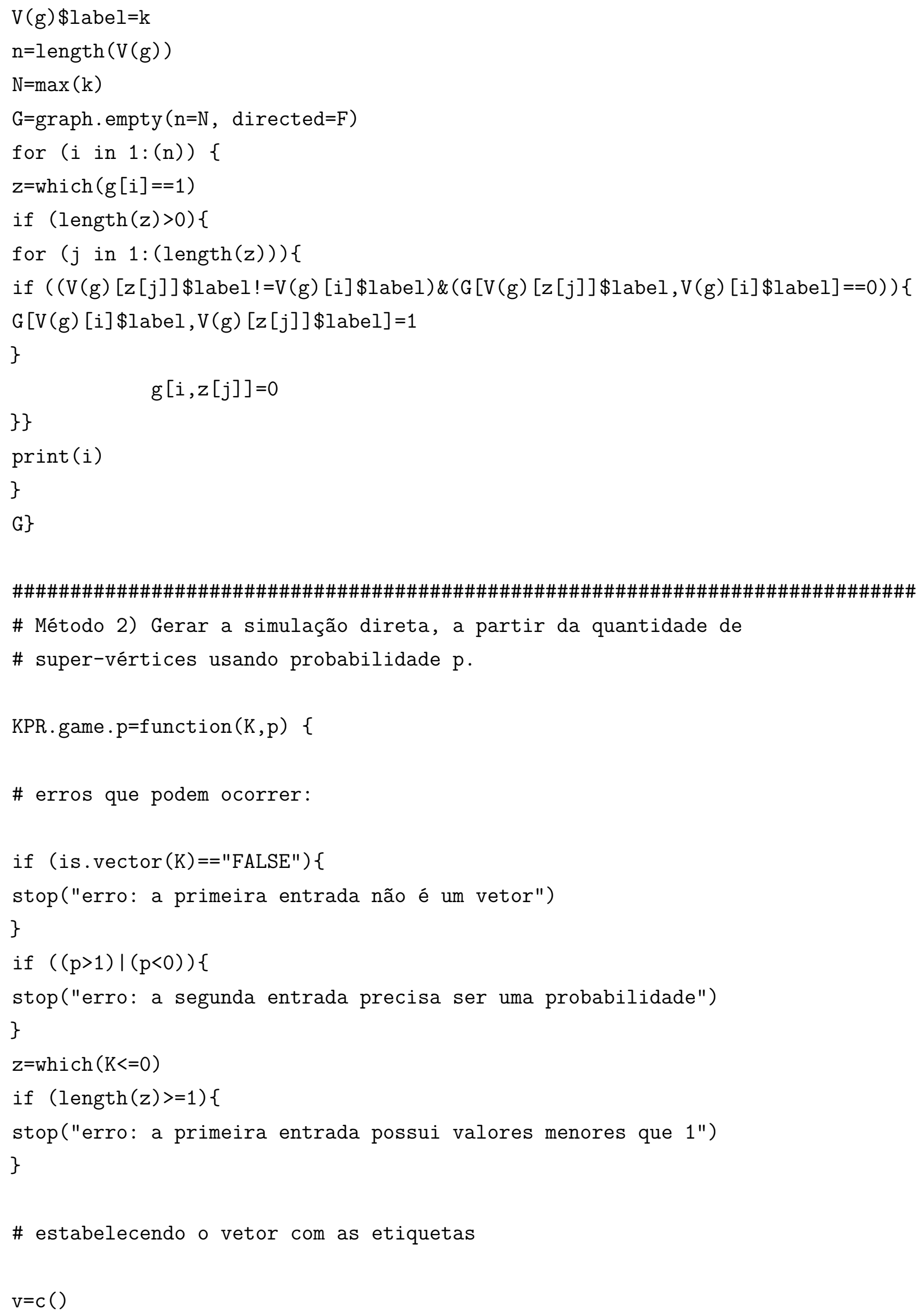




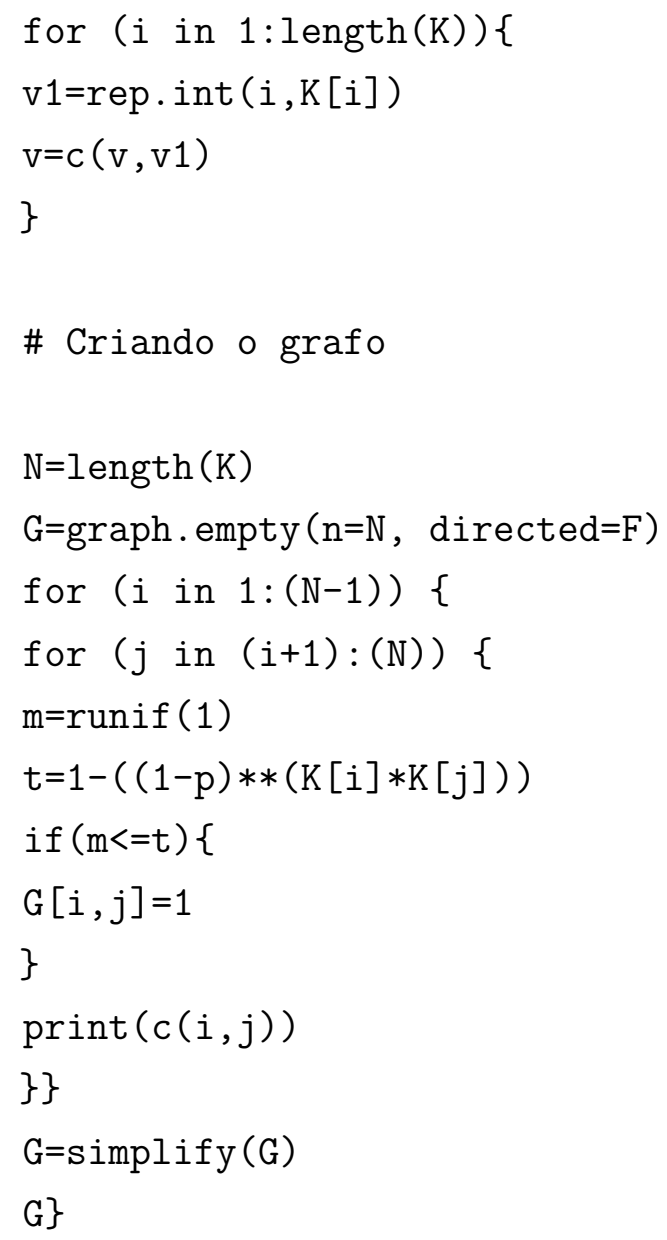




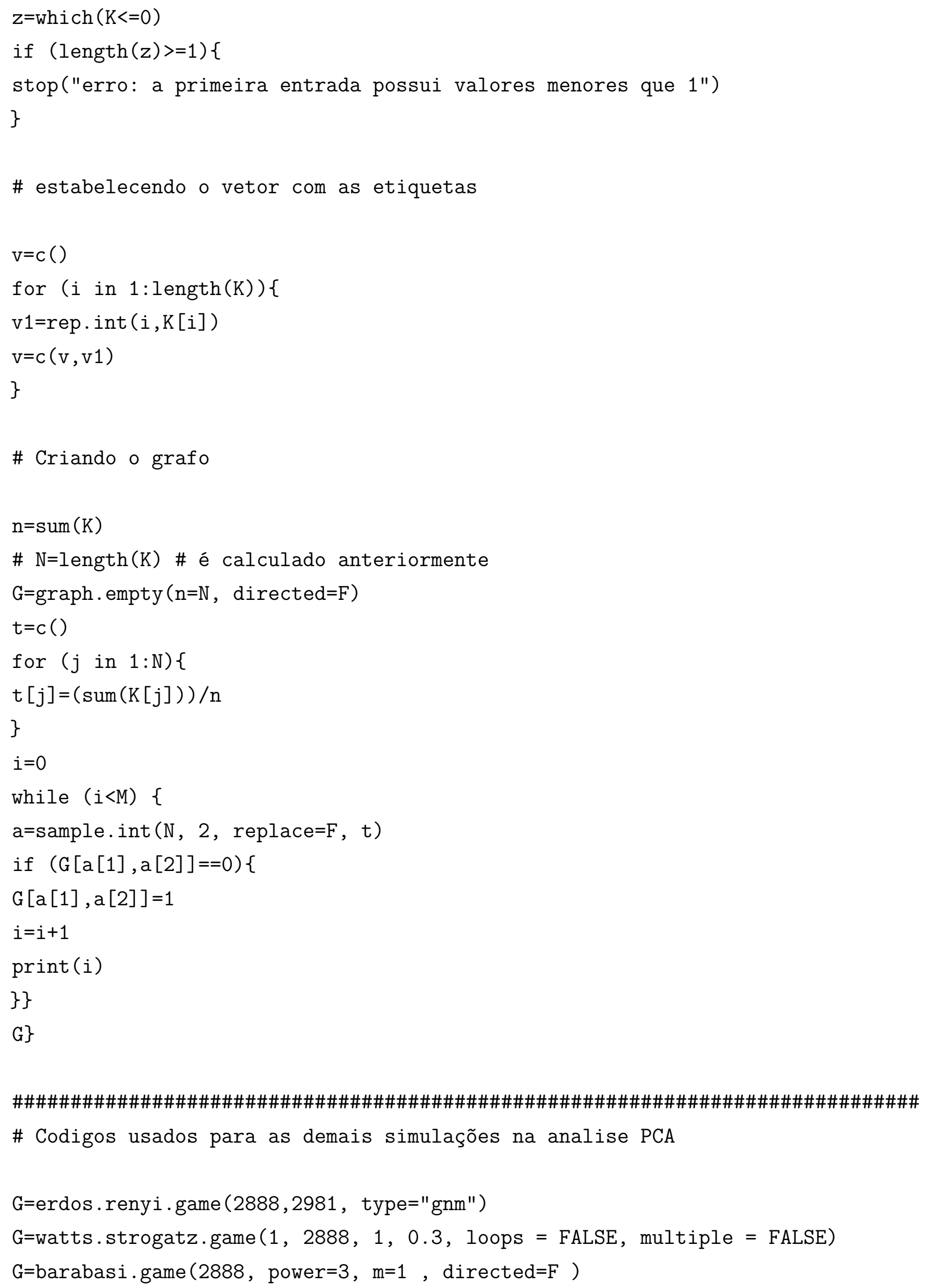

